\title{
CHANGES IN THE DYNAMICAL BEHAVIOR OF NONLINEAR SYSTEMS INDUCED BY NOISE
}

\author{
P.S. LANDA ${ }^{\mathrm{a}}$, P.V.E. McCLINTOCK ${ }^{\mathrm{b}}$ \\ a Department of Physics, Lomonosov Moscow State University, 119899 Moscow, Russia \\ ${ }^{\mathrm{b}}$ Department of Physics, Lancaster University, Lancaster LA1 4YB, UK
}

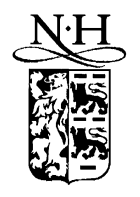

ELSEVIER 


\title{
Changes in the dynamical behavior of nonlinear systems induced by noise
}

\author{
Polina S. Landa ${ }^{\mathrm{a}}$, P.V.E. McClintock ${ }^{\mathrm{b}, *}$ \\ ${ }^{a}$ Department of Physics, Lomonosov Moscow State University, 119899 Moscow, Russia \\ ${ }^{\mathrm{b}}$ Department of Physics, Lancaster University, Lancaster LA1 4YB, UK
}

Received April 1999; editor: I. Procaccia

\section{Contents}

1. Introduction

2. Fluctuational transitions of nonlinear systems from one stable steady state to another

2.1. Elements of the theory of fluctuational transitions

2.2. Applications of the theory of fluctuational transitions to the problems of intermittency

3. Noise-induced transport of Brownian particles (stochastic ratchets)

3.1. Noise-induced transport of light Brownian particles in a viscous medium with a saw-tooth potential
4

4

4

12

19

21
3.2. The effect of the potential shape

3.3. The effect of the particle mass

4. Noise-induced phase transitions in nonlinear oscillators

4.1. Noise-induced multistability and multimodality

4.2. Noise-induced oscillations

5. Conclusions

Appendix A. Derivation of the approximate equation for the one-dimensional probability density

References

\begin{abstract}
Weak noise acting upon a nonlinear dynamical system can have far-reaching consequences. The fundamental underlying problem - that of large deviations of a nonlinear system away from a stable or metastable state, sometimes resulting in a transition to a new stationary state, in response to weak additive or multiplicative noise - has long attracted the attention of physicists. This is partly because of its wide applicability, and partly because it bears on the origins of temporal irreversibility in physical processes. During the last few years it has become apparent that, in a system far from thermal equilibrium, even small noise can also result in qualitative change in the system's properties, e.g., the transformation of an unstable equilibrium state into a stable one, and vice versa, the occurrence of multistability and multimodality, the
\end{abstract}

\footnotetext{
* Corresponding author.

E-mail address: mcclintock@lancaster.ac.uk (P.V.E. McClintock)
} 
appearance of a mean field, the excitation of noise-induced oscillations, and noise-induced transport (stochastic ratchets). A representative selection of such phenomena is discussed and analyzed, and recent progress made towards their understanding is reviewed. (C) 2000 Elsevier Science B.V. All rights reserved.

PACS: 05.10.Gg; 05.40. - a; 05.60. - k

Keywords: Fluctuational transitions; Stochastic ratchets; Noise-induced phase transitions 


\section{Introduction}

The problems of large deviations of nonlinear systems away from an equilibrium state, and transitions to a new state, in response to weak noise, that can be either of internal or of external origin, have long attracted the close attention of physicists, in part because these problems are associated with irreversibility of physical processes. In the last few years it has emerged that, in systems far from thermal equilibrium, weak noise can produce qualitative change in the properties of a system, e.g., the transformation of an unstable equilibrium state to a stable one and vice versa [1], the occurrence of multistability or multimodality [2,3], the appearance of a mean field [4-7], the excitation of noise-induced oscillations [8-11], the occurrence of a peculiar kind of resonance (so-called stochastic resonance) [12-19], a possibility of one-directional motion (net current) under the action of zero average forces (so-called stochastic ratchet) $[20-22,24,18]$ and so on. Many such effects have been demonstrated in analogue electronic experiments - which in turn provided the stimulus for further developments in the theory [23]. The aim of this review is to provide an accessible introduction to such phenomena. We proceed by reviewing the basis of the theory, and then consider some illustrative examples of current interest. In Section 2, we outline the theory of fluctuational transitions and discuss how it can be applied to the problem of intermittency, in which the dynamical properties of the system change in a seemingly random way between regular and chaotic behavior. The theory is applied to the Brownian ratchet problem in Section 3, where we consider the physical basis of noise-induced transport and derive explicit expressions for the flow in several different limits. In particular, we consider diffusion in a saw-tooth potential with an additional regular force, random modulation of the potential barrier height, the effect of an additional random force with a large correlation time, the influence of the shape of the potential, and the effect of the mass of the diffusing particle. Another phenomenon in which the physical behavior of the system is radically changed by the presence of noise is that of the noise-induced phase transition. This is discussed in Section 4 in relation to noise-induced multistability, multimodality, and noise-induced oscillations. The formalism is applied to several examples of topical interest including a pendulum with a randomly vibrated axis of suspension, a generic oscillator with quadratic nonlinearity (which undergoes a noise-induced phase transition under the action of additive noise), a model of childhood epidemics, and the Bonhoeffer-van der Pol oscillator. We draw the ideas together and offer some conclusions in Section 5. A formal derivation of the approximate equation for the one-dimensional probability density is provided in the appendix.

\section{Fluctuational transitions of nonlinear systems from one stable steady state to another}

\subsection{Elements of the theory of fluctuational transitions}

The problem of how transitions occur from one stable state of a system to another under influence of weak noise can be reduced to the statistical problem of the probability of the first attainment of a boundary by a Brownian particle moving in a given force field [25-27]. Several examples of such problems, as applied to systems of different physical origin, were considered, e.g., in [27-39]. The best known of these is [28], in which the problem was solved for a double-well oscillator in order to estimate the rates of chemical reactions. All of the systems considered in the 
papers cited belong to the class of nonlinear oscillators with two or more stable steady states. In the absence of fluctuations, the system, being in one of these states, cannot pass to one of the other states without external action of some kind. In the presence of weak noise, however, the system executes small random oscillations in the vicinity of one of the steady states and, from time to time, undergoes a transition to a different state. If the noise is sufficiently weak, such transitions occur only very rarely. Thus that the system remains in the vicinity of the corresponding stable state over a long period, and the probability distribution consequently has a chance to reach its stationary value. First we consider systems for which one can obtain, exactly or approximately, a single first-order differential equation with a random source describing the behavior of a certain variable $z$ characterizing the motion of the system.

As an example, let us consider a double-well oscillator with a sufficiently small (in comparison with its natural frequency) damping factor [27]. Its equation of motion can be written as

$$
\ddot{x}+\gamma \dot{x}+F(x)=\xi(t),
$$

where $\xi(t)$ is a random process. In the particular case when $F(x)=-a x+b x^{3}$, Eq. (2.1) coincides with that considered by Kramers [28]. If the damping factor $\gamma$ and the intensity of the noise $\xi(t)$ are sufficiently small then the oscillator energy, which is described by

$$
E=\left(\dot{x}^{2} / 2\right)+U(x),
$$

where $U(x)=\int_{0}^{x} F(x) \mathrm{d} x$, is a slowly varying function. The stable steady states correspond to minima of the function $U(x)$, and the unstable ones correspond to maxima of this function. A transition from one stable steady state to another can occur when $U(x)$ attains its maximal value. Multiplying both sides of Eq. (2.1) by $\dot{x}$ we obtain the following exact equation for $E$ :

$$
\dot{E}=-\gamma \dot{x}^{2}+\dot{x} \xi(t) \text {. }
$$

So, combining (2.2) and (2.3) we obtain the two stochastic equations

$$
\begin{aligned}
& \dot{x}=\sqrt{2(E-U(x))}, \\
& \dot{E}=-2 \gamma(E-U(x))+\sqrt{2(E-U(x))} \xi(t) .
\end{aligned}
$$

In the case when the damping constant $\gamma$, and the intensity and correlation time of the noise, are all sufficiently small ${ }^{1}$ the two-dimensional Fokker-Planck equation corresponding to the Langevin equations (2.4) can [26] be reduced to the one-dimensional equation

$$
\frac{\partial w(E, t)}{\partial t}=\frac{\partial}{\partial E}\left(\left(\gamma \omega(E) J(E)-\frac{\kappa}{2}\right) w\right)+\frac{\kappa}{2} \frac{\partial^{2}}{\partial E^{2}}(\omega(E) J(E) w),
$$

where

$$
J(E)=\frac{1}{\pi} \int_{x_{\min }}^{x_{\max }} \sqrt{2(E-U(x))} \mathrm{d} x
$$

\footnotetext{
${ }^{1}$ The condition for the smallness of the noise intensity can be written as $\left\langle\left(\int_{t}^{t+T} \dot{x} \xi(t) \mathrm{d} t\right)^{2}\right\rangle^{1 / 2} \ll U_{\max }$, where $T$ is an interval of time of the order of the mean period of oscillations in the vicinity of the stable steady state of interest.
} 
is the action, and

$$
\omega(E)=\pi\left(\int_{x_{\min }}^{x_{\max }} \frac{\mathrm{d} x}{\sqrt{2(E-U(x))}}\right)^{-1}
$$

is the oscillation frequency for a fixed value of the energy $E ; x_{\min }$ and $x_{\max }$ are the extreme values taken by $x$ during the oscillations, and they are approximately equal to the roots of the equation $U(x)=E ; \kappa$ is the spectral density of the random process $\xi(t)$ at a certain characteristic oscillation frequency. ${ }^{2}$ It is evident that the following Langevin equation can be related to the Fokker-Planck equation (2.5):

$$
\dot{E}=-\gamma \omega(E) J(E)+\frac{\kappa}{2}\left(1-\frac{1}{2} \frac{\mathrm{d}(\omega(E) J(E))}{\mathrm{d} E}\right)+\zeta(E, t),
$$

where $\zeta(E, t)$ is white noise of zero mean and intensity $K(E)=\omega(E) J(E) \kappa$. So, let us consider the equation

$$
\dot{z}=\varphi(z, \xi),
$$

where $\xi(t)$ is sufficiently wide-band ${ }^{3}$ noise, and the mean value of the right-hand side $\langle\varphi(z, \xi)\rangle \equiv f(z)$ vanishes at the points $z=z_{0}$ and $z=z_{1}$ and is negative for $z_{0}<z<z_{1}$. This implies that the point $z_{0}$ is a stable steady state and that $z_{1}$ is an unstable steady state.

As shown in [26], under the condition of the smallness of the noise correlation time indicated above, $\varphi(z, \xi)$ can be represented as $\varphi(z, \xi)=F(z)+\zeta(z, t)$, where $F(z)=f(z)+K^{\prime}(z)$,

$$
K^{\prime}(z)=\int_{-\infty}^{0}\left(\left\langle\frac{\partial \varphi(z, \xi(t))}{\partial z} \varphi(z, \xi(t+\tau))\right\rangle-f(z) \frac{\mathrm{d} f(z)}{\mathrm{d} z}\right) \mathrm{d} \tau,
$$

and $\zeta(z, t)$ is zero-mean white noise of intensity

$$
K(z)=2 \int_{-\infty}^{0}\left(\langle\varphi(z, \xi(t)) \varphi(z, \xi(t+\tau))\rangle-f^{2}(z)\right) \mathrm{d} \tau .
$$

Because $z$ can then be considered as a Markov process, we can use the Fokker-Planck equation for the probability density $w(z, t)$ :

$$
\frac{\partial w}{\partial t}=-\frac{\partial}{\partial z}(F(z) w(z, t))+\frac{1}{2} \frac{\partial^{2}}{\partial z^{2}}(K(z) w(z, t)) .
$$

The stationary solution of Eq. (2.8) satisfying the condition for zero probability flux is

$$
w_{\mathrm{st}}(z)=\frac{C}{K(z)} \exp (-\psi(z))
$$

\footnotetext{
${ }^{2}$ We assume that $\kappa$ does not depend on $E$.

${ }^{3} \mathrm{We}$ assume that the correlation time of the noise is small in comparison with the duration of transient processes in the system which we denote $\tau_{\text {tr }}$, i.e., the width of the noise band is much more than $1 / \tau_{\text {tr }}$.
} 
where the constant $C$ is determined from the normalization condition, and

$$
\psi(z)=-2 \int_{z_{0}}^{z}(F(z) / K(z)) \mathrm{d} z
$$

It is easy to verify that, for small noise intensity $K(z)$, the function $w_{\mathrm{st}}(z)$ peaks at the points corresponding to stable steady states, in particular, at the point $z_{0}$.

Let us calculate the probability for the passage of the system from a certain point $z^{\prime}$ lying in the range from $z_{2}$ to $z_{1}$, where $z_{2} \leq z_{0}$, through the boundary $z=z_{1}$. Clearly, for sufficiently small noise intensity, the probability of reaching the boundary must be independent of the initial point $z^{\prime}$, provided only that this point is not located too close to the boundary. Let us denote a solution of Eq. (2.8), satisfying the conditions

$$
w\left(z, z^{\prime}, 0\right)=\delta\left(z-z^{\prime}\right), \quad w\left(z_{1}, z^{\prime}, t\right)=0,
$$

by $w\left(z, z^{\prime}, t\right)$. Then the probability that $z$ does not attain the boundary $z=z_{1}$ in a time $t$ is

$$
P\left(t, z^{\prime}\right)=\int_{z_{2}}^{z_{1}} w\left(z, z^{\prime}, t\right) \mathrm{d} z
$$

One method of calculating $P\left(t, z^{\prime}\right)$ was suggested in [27]. The probability density $w\left(z, z^{\prime}, t\right)$ as a function of $z^{\prime}$ is described by the equation conjugate to Eq. (2.8), namely

$$
\frac{\partial w\left(z, z^{\prime}, t\right)}{\partial t}=F\left(z^{\prime}\right) \frac{\partial w\left(z, z^{\prime}, t\right)}{\partial z^{\prime}}+\frac{K\left(z^{\prime}\right)}{2} \frac{\partial^{2} w\left(z, z^{\prime}, t\right)}{\partial z^{\prime 2}} .
$$

Integrating Eq. (2.12) over $z$ from $z_{2}$ to $z_{1}$, and taking account of (2.11), we obtain an equation for the probability $P\left(t, z^{\prime}\right):^{4}$

$$
\frac{\partial P(t, z)}{\partial t}=F(z) \frac{\partial P(t, z)}{\partial z}+\frac{K(z)}{2} \frac{\partial^{2} P(t, z)}{\partial z^{2}} .
$$

Let us represent $\partial P(t, z) / \partial t$ in terms of the characteristic function

$$
\Theta(\mathrm{i} v, z)=-\int_{0}^{\infty} \frac{\partial P(t, z)}{\partial t} \mathrm{e}^{\mathrm{i} v t} \mathrm{~d} t .
$$

Expanding both sides of expression (2.14) as a power series in $\mathrm{i} v$ we obtain

$$
\Theta(\mathrm{i} v, z)=\sum_{k=0}^{\infty} \frac{(\mathrm{i} v)^{k}}{k !} m_{k}(z),
$$

where

$$
m_{k}(z)=-\int_{0}^{\infty} t^{k} \frac{\partial P(t, z)}{\partial t} \mathrm{~d} t
$$

is the $k$ th moment of the attainment time. Because $P(\infty, z)=0$ and $P(0, z)=1$, then $m_{0}(z)=P(0, z)-P(\infty, z)=1$. Differentiating both sides of Eq. (2.13) with respect to $t$, multiplying

\footnotetext{
${ }^{4}$ Below we substitute unprimed $z$ in place of primed $z^{\prime}$.
} 
by $\mathrm{e}^{\mathrm{i} v t}$ and integrating over $t$ from 0 to $\infty$, we obtain the following equation for the characteristic function $\Theta(\mathrm{i} v, z)$ :

$$
-\mathrm{i} v \Theta=F(z) \frac{\partial \Theta}{\partial z}+\frac{K(z)}{2} \frac{\partial^{2} \Theta}{\partial z^{2}} .
$$

Substituting (2.15) in Eq. (2.17) we can obtain equations for all of the moments of the attainment time. In particular, for the mean first attainment time $M(z) \equiv m_{1}(z)$ we find

$$
\frac{K(z)}{2} \frac{\mathrm{d}^{2} M}{\mathrm{~d} z^{2}}+F(z) \frac{\mathrm{d} M}{\mathrm{~d} z}+1=0 .
$$

This equation, as well as Eq. (2.13), was first derived in [25]. Therefore in the Russian mathematical literature these equations are known as the first and the second Pontryagin equations, respectively.

To solve Eq. (2.18) we must set two boundary conditions. One of these is immediately evident: it is

$$
M\left(z_{1}\right)=0 .
$$

The second boundary condition depends on the character of the boundary $z=z_{2}$ [27]. If it is perfectly reflecting, and the requirements that $K\left(z_{2}\right) \neq 0,\left|f\left(z_{2}\right)\right|<\infty$ and $z_{2} \neq-\infty$ are fulfilled, then $\mathrm{d} M /\left.\mathrm{d} z\right|_{z=z_{2}}=0$ [27]. If one of these requirements is not fulfilled, however, then we must use as the second boundary condition the requirement of boundedness of the function $M(z)$ at the point $z=z_{2}$. A solution of Eq. (2.18) satisfying the condition (2.19) is [25]

$$
M(z)=2 \int_{z}^{z_{1}} \int_{z_{2}}^{z^{\prime}} \frac{1}{K(z)} \exp (-\psi(z)) \exp \left(\psi\left(z^{\prime}\right)\right) \mathrm{d} z \mathrm{~d} z^{\prime}+C \int_{z}^{z_{1}} \exp \left(\psi\left(z^{\prime}\right)\right) \mathrm{d} z^{\prime},
$$

where the constant $C$ is determined from the second boundary condition. In all examples considered in [29-31,27] the second boundary condition causes $C$ to be equal to zero.

In the case of sufficiently weak noise, for $C=0$, expression (2.20) can [27] be reduced approximately to

$$
M(z) \approx 2 \int_{z_{2}}^{z_{1}} \frac{1}{K(z)} \exp (-\psi(z)) \mathrm{d} z \int_{z_{2}}^{z_{1}} \exp (\psi(z)) \mathrm{d} z .
$$

If the conditions

$$
\left|z_{1}-z_{0}\right| \gg \sqrt{-Q\left(z_{0}\right)}, \quad\left|z_{2}-z_{0}\right| \gg \sqrt{-Q\left(z_{0}\right)}, \quad\left|z-z_{1}\right| \gg \sqrt{Q\left(z_{1}\right)},
$$

where

$$
Q(z)=\frac{1}{2}\left\{\frac{\mathrm{d}}{\mathrm{d} z}\left(\frac{F(z)}{K(z)}\right)\right\}^{-1},
$$

are fulfilled, the integrals in expression (2.21) can be calculated approximately by using a method similar to the saddle-point technique. We thus obtain

$$
M(z) \approx \pi \frac{\sqrt{-Q\left(z_{0}\right) Q\left(z_{1}\right)}}{K\left(z_{0}\right)} \exp \left(\psi\left(z_{1}\right)-\psi\left(z_{0}\right)\right) .
$$


We see from (2.23) that, in the approximation considered, the mean first passage time is independent of $z$ and exponentially dependent of the potential barrier height characterized by the difference $\psi\left(z_{1}\right)-\psi\left(z_{0}\right)$. If the second condition of (2.22) is not fulfilled, e.g., $z_{2}=z_{0}$, then an approximate calculation of the integrals in expression (2.21) can be performed in another way. As an example, let us consider Eq. (2.6). For this equation expression (2.21) takes the form:

$$
M(E) \approx \frac{2}{\kappa} \int_{E_{0}}^{E_{1}} \frac{1}{\omega(E)} \exp \left(-\frac{2 \gamma}{\kappa} E\right) \mathrm{d} E \int_{E_{0}}^{E_{1}} \frac{1}{J(E)} \exp \left(\frac{2 \gamma}{\kappa} E\right) \mathrm{d} E .
$$

Taking account of the fact that $\exp (\mp 2(\gamma / \kappa) E)$ have their largest values for $E=E_{0}$ and $E=E_{1}$, respectively, and decline rapidly for small $\kappa\left(\kappa \ll \gamma\left(E_{1}-E_{0}\right)\right)$, in the first integral of (2.24) we can substitute $\omega\left(E_{0}\right)$ in place of $\omega(E)$ and in the second integral we can substitute $J\left(E_{1}\right)$ in place of $J(E)$. In so doing we obtain

$$
M(E) \approx \frac{\kappa}{2 \gamma^{2}} \frac{1}{\omega\left(E_{0}\right) J\left(E_{1}\right)} \exp \left(\frac{2 \gamma}{\kappa}\left(E_{1}-E_{0}\right)\right),
$$

where $\omega\left(E_{0}\right)$ is the frequency of small oscillations around the stable steady state corresponding to $E=E_{0}$. We note that a formula similar to (2.25) was obtained by Kramers [28] and is well known as the Kramers formula.

The value of $M$ is equal to the mean time at which $z$ first attains the boundary $z=z_{1}$. If the potential $\psi(z)$ at this boundary has a smooth maximum, then the probability of passing through the boundary $(p)$ is equal to the probability $(1-p)$ of returning back again, i.e., $p=1 / 2$. Hence the mean time of the passage through the boundary $T$ has to be equal to $2 M$. As can be shown, if $p \neq 1-p$ then

$$
T=M / p \text {. }
$$

We now consider another method of calculating the probability $P(t, z)$ and the mean first attainment time. It is based on solving the nonstationary Fokker-Planck equation (2.8). For the most part this equation cannot be solved exactly. However, in the case of sufficiently small noise, methods for obtaining approximate solutions of Eq. (2.8) are known. One of them was suggested in [27]. Because Eq. (2.8) is linear, its solution, satisfying the boundary condition $w\left(z_{1}, t\right)=0$ can be represented as

$$
w(z, t)=\sum_{n=0}^{\infty} \mathrm{e}^{-\lambda_{n} t} w_{n}(z),
$$

where $w_{n}(z)$ is $n$th eigenfunction described by the equation

$$
\frac{1}{2} \frac{\mathrm{d}^{2}}{\mathrm{~d} z^{2}}\left(K(z) w_{n}(z)\right)-\frac{\mathrm{d}}{\mathrm{d} z}\left(F(z) w_{n}(z)\right)+\lambda_{n} w_{n}(z)=0
$$

with the boundary condition

$$
w_{n}\left(z_{1}\right)=0 .
$$

In the case that the noise is weak passages through the boundary are rare and, as a consequence, the least eigenvalue $\lambda_{0}$ is small, whereas the other eigenvalues are vastly greater. Therefore, after a characteristic time that is short in comparison with $1 / \lambda_{0}$ but long in comparison with 
$1 / \lambda_{1}, 1 / \lambda_{2}, \ldots$, the main contribution to solution (2.27) will come from the first eigenfunction $w_{0}(z)$ associated with the eigenvalue $\lambda_{0}$. So, we can write approximately

$$
w(z, t) \approx \mathrm{e}^{-\lambda_{0} t} w_{0}(z) .
$$

The normalization condition for $w_{0}(z)$ we set in the form

$$
\int_{z_{2}}^{z_{1}} w_{0}(z) \mathrm{d} z=1
$$

It follows from (2.11), (2.30), and (2.31) that

$$
P(t, z)=\mathrm{e}^{-\lambda_{0} t} .
$$

The fact that $P(t, z)$ does not depend on $z$ is associated with our having used from the outset the small noise approximation. From (2.16) and (2.32) we obtain that $M=1 / \lambda_{0}$.

For calculating $\lambda_{0}$ we integrate Eq. (2.28) for $n=0$ over $z$ from $z_{2}$ to $z_{1}$. Using the normalization condition (2.31) we find

$$
\lambda_{0}=G\left(z_{1}\right)-G\left(z_{2}\right),
$$

where

$$
G(z)=F(z) w_{0}(z)-\frac{1}{2} \frac{\mathrm{d}}{\mathrm{d} z}\left(K(z) w_{0}(z)\right)
$$

is the probability flux. If the boundary $z=z_{2}$ is perfectly reflecting, and the conditions (2.22) are fulfilled, then $G\left(z_{2}\right)=0$. In the case when $z_{2}=z_{0}$ we can also put $G\left(z_{2}\right) \approx 0$ because, in the vicinity of $z=z_{0}$, the probability density $w_{0}(z)$ coincides closely in shape with the stationary distribution (2.9) for which $G=0$. Taking account of the boundary condition (2.29) we find

$$
G\left(z_{1}\right)=-\left.\frac{1}{2} \frac{\mathrm{d}}{\mathrm{d} z}\left(K(z) w_{0}(z)\right)\right|_{z=z_{1}} .
$$

It follows from (2.33) and (2.35) that, for calculating $\lambda_{0}$, a knowledge of the solution of Eq. (2.28) for $n=0$ in the vicinity of the boundary $z=z_{1}$ is sufficient. Because the value of $w_{0}(z)$ in the vicinity of $z=z_{1}$ is very small, owing to the boundary condition (2.29), we can neglect the term $\lambda_{0} w_{0}(z)$ there. Moreover, we can neglect this term for all values of $z$ because, away from the boundary, as mentioned above, the probability density $w_{0}(z)$ has to coincide in shape with the stationary distribution (2.9). Thus, for calculating $w_{0}(z)$ we can use the stationary Fokker-Planck equation with the boundary condition (2.29) and the normalization condition (2.31).

The first integral of the stationary Fokker-Planck equation in view of (2.33) is

$$
F(z) w_{0}(z)-\frac{1}{2} \frac{\mathrm{d}}{\mathrm{d} z}\left(K(z) w_{0}(z)\right)=\lambda_{0} .
$$

Solving Eq. (2.36) with the boundary condition (2.29) we find

$$
w_{0}(z)=\frac{2 \lambda_{0}}{K(z)} \exp (-\psi(z)) \int_{z}^{z_{1}} \exp \left(\psi\left(z^{\prime}\right)\right) \mathrm{d} z^{\prime},
$$


where $\psi(z)$ is defined by expression (2.10). The value of $\lambda_{0}$ is found from the normalization condition (2.31):

$$
\lambda_{0}^{-1}=M=2 \int_{z_{2}}^{z_{1}} \frac{1}{K(z)} \exp (-\psi(z))\left\{\int_{z}^{z_{1}} \exp \left(\psi\left(z^{\prime}\right)\right) \mathrm{d} z^{\prime}\right\} \mathrm{d} z .
$$

By reasoning as above we can remove the term in braces from the first integral by putting $z=z_{2}$. In doing so we obtain an expression coinciding with (2.21). Another approximate method for solving the nonstationary Fokker-Planck equation (2.8) in the case of sufficiently small noise is based on a technique similar to the WKB method. It was applied to the indicated problem in, for example, $[40,41]$. The results coincide with those set forth above.

To conclude this section, let us consider in more detail the question of the most probable trajectory $z_{\text {opt }}(t)$ along which a fluctuational transition will occur. In recent years this problem has attracted considerable interest from many researchers (see, e.g., [42-48]). Let a system be described by the equations

$$
\dot{\boldsymbol{y}}=\boldsymbol{F}(\boldsymbol{y})+\boldsymbol{\xi}(\boldsymbol{y}, t),
$$

where $\boldsymbol{\xi}(\boldsymbol{y}, t)$ is white noise of zero mean and intensity $K(\boldsymbol{y})$, and $\boldsymbol{F}(\boldsymbol{y})$ vanishes at the points $\boldsymbol{y}=\boldsymbol{y}_{0}$ and $\boldsymbol{y}=\boldsymbol{y}_{1}$, i.e., $\boldsymbol{y}_{0}$ and $\boldsymbol{y}_{1}$ are singular points. We assume that the point $\boldsymbol{y}_{0}$ is stable and that the point $\boldsymbol{y}_{1}$ is unstable. It was shown in [42] that, in the case when $K(\boldsymbol{y})$ is independent of $\boldsymbol{y}$, the most probable trajectory $\boldsymbol{y}_{\text {opt }}(t)$ can be determined as a partial solution of the auxiliary Hamilton equations

$$
\dot{\boldsymbol{y}}=\mathrm{\partial H} / \mathrm{\partial} \boldsymbol{p}, \quad \dot{\boldsymbol{p}}=-\mathrm{\partial} H / \partial \boldsymbol{y},
$$

where

$$
H(\boldsymbol{y}, \boldsymbol{p})=\boldsymbol{p} \boldsymbol{F}(\boldsymbol{y})+\left(\boldsymbol{p}^{2} / 2\right) K(\boldsymbol{y})
$$

is a so-called Wentzel-Freidlin Hamiltonian. The required solution has to satisfy the condition $H=0$. It is easily shown that Eqs. (2.40) are valid if $K(\boldsymbol{y})$ depends on $\boldsymbol{y}$ as well. In particular, for a one-dimensional system described by Eq. (2.7) with $\varphi(z, \xi)=F(z)+\zeta(z, t)$ Eqs. (2.40) become

$$
\dot{z}=\partial H / \partial p, \quad \dot{p}=-\partial H / \partial z,
$$

where

$$
H(z, p)=p F(z)+\left(p^{2} / 2\right) K(z) .
$$

From the condition $H=0$ we find $p=2 F(z) / K(z)$. Substituting this expression into the first equation of (2.42) we obtain

$$
\dot{z}=-F(z),
$$

i.e., the most probable trajectory $z_{\mathrm{opt}}(t)$ along which the representative point moves away from a stable singular point $z=z_{0}$ coincides with the incoming trajectory of the corresponding dynamical system arriving at this point. 
A simple explanation of this result for the one-dimensional case can be given as follows. Because the function

$$
\frac{1}{K(z)} \int_{z}^{z_{1}} \exp \left(\psi\left(z^{\prime}\right)\right) \mathrm{d} z^{\prime}
$$

in expression (2.37) for the eigenfunction $w_{0}(z)$ varies more slowly than $\exp (-\psi(z))$, the probability density $w(z, t)$ is maximal for $z(t)$ satisfying the minimization condition of $\Psi(t)=\psi(z(t))$. This quantity can be considered as a classical action characterizing the motion along different trajectories outgoing, for $t=t_{0}$, from a common point and incoming, for a certain instant $t$, to different points. As is known from classical mechanics [49], this action is minimal for trajectories obeying the Hamilton equations (2.42), where the Hamiltonian $H(z, p, t)$ is associated with the action $\Psi(t)$ by the Hamilton-Jacobi equation

$$
\partial \Psi / \partial t+H(z, p)=0 .
$$

Let us find the action corresponding to a Hamiltonian of form (2.43). It is known [49] that the action $S(t)$ is determined by

$$
S(t)=\int_{t_{0}}^{t} L \mathrm{~d} t,
$$

where $L$ is the Lagrangian which is associated with the Hamiltonian by the relation

$$
L=p \dot{z}-H .
$$

Let us now rewrite expression (2.10) in the form

$$
\psi(z(t))=\Psi(t)=\int_{t_{0}}^{t} \tilde{L} \mathrm{~d} t,
$$

where $\tilde{L}=-2 \dot{z} F(z) / K(z)$. Comparing (2.46) with (2.48) we see that $S(t)=\Psi(t)$ if $L=\tilde{L}$. It follows from (2.47) and (2.43) that the latter condition is fulfilled if $H=0$. Hence, for the trajectory $z(t)$ described by Eq. (2.44) the quantity $\Psi(t)$ is minimal and therefore the probability density $w(z, t)$ is indeed maximal. It should be noted that the optimal trajectories have recently been observed and studied in analogue electronic experiments on nonlinear oscillators $[44,46,47,23]$.

\subsection{Applications of the theory of fluctuational transitions to the problems of intermittency}

\subsubsection{Intermittency as a result of a tangent bifurcation}

It is well known that one possible route for the loss of stability of regular motion and the onset of chaos in dynamical systems is the fusion of a stable steady state with an unstable one, with the subsequent disappearance of both of these states. In certain conditions after this bifurcation, which is often said to be tangential, the motion of the system exhibits the property of intermittency (see, for example, [50-52]). This property implies that in the system phase space the representative point is "walking" over prolonged periods within a small vicinity of the vanished states. The motion of the system corresponding to such walking is nearly regular (laminar phases). These long intervals of nearly regular motion alternate with short irregular bursts (turbulent phases). Away from the 
bifurcation point the duration of the laminar phases decreases and that of the turbulent ones increases until, finally, the laminar phases disappear altogether. The dependence of the mean duration of the laminar phases on the excess of the bifurcation parameter beyond its critical value can be evaluated by using a model map (see, for example, [53-55,35,36,52]). If the system is acted upon by weak noise then the mean duration of the laminar phases is also dependent on the noise intensity. We will concentrate on the results of $[35,52]$, in which the theory of the passage through a boundary was used.

Let us consider a system described by a one-dimensional map that can be presented as shown in Fig. 1 close to the bifurcation point. At the bifurcation point a stable fixed point of the map, $M_{1}$, fuses with an unstable one, $M_{2}$, and disappears. We set the bifurcation parameter $\varepsilon$ equal to zero as
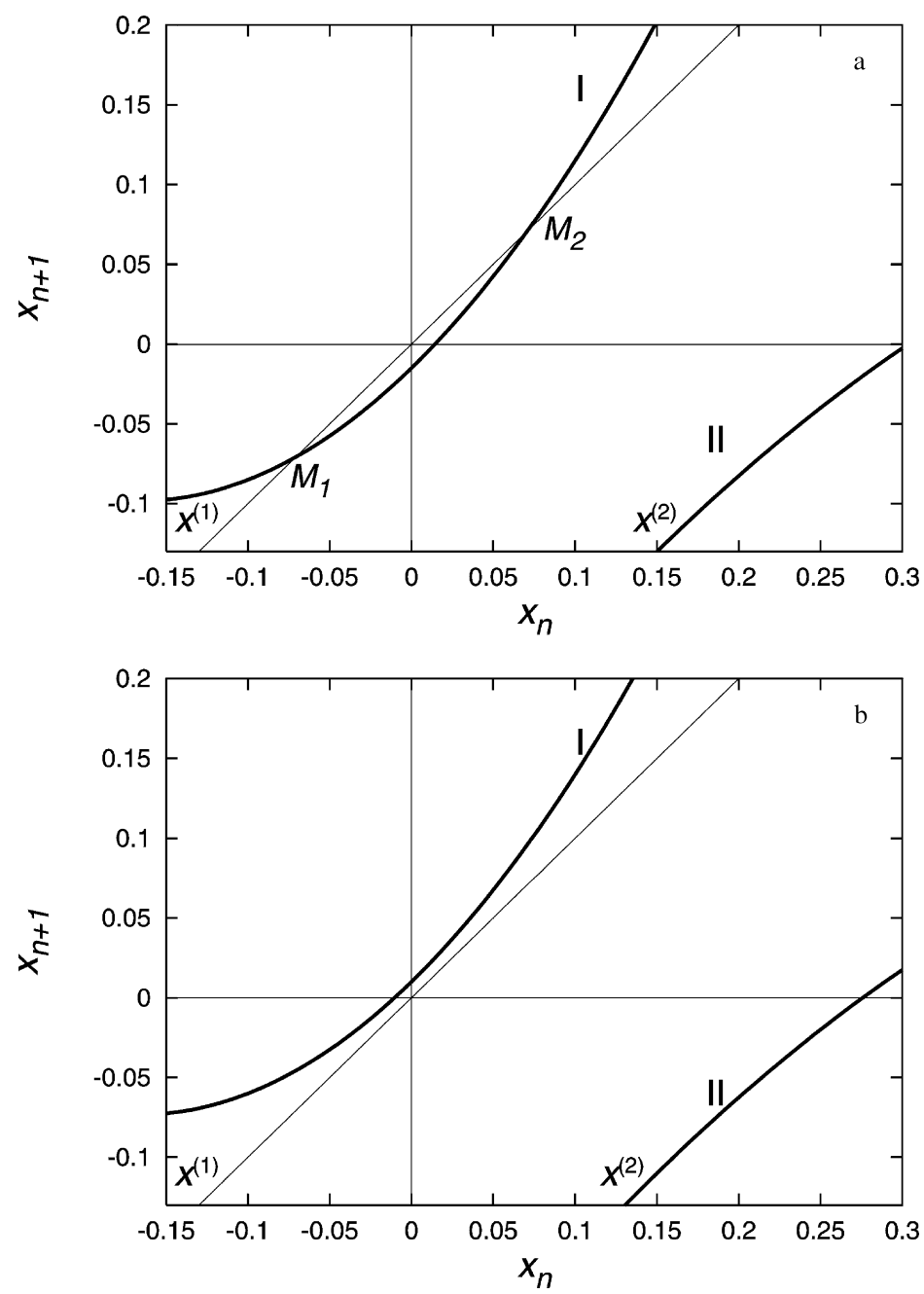

Fig. 1. Sketch of a model map close to the transition through intermittency: (a) before the transition; and (b) after the transition. 
the map touches the bisectrix. For sufficiently small $\varepsilon,\left|x^{(1)}\right|$ and $\left|x^{(2)}\right|^{5}$ the part of the map labeled I can be approximated as

$$
x_{n+1}=\varepsilon+x_{n}+a x_{n}^{q},
$$

where $q$ is an even number. It is evident that laminar phases are associated with motion of the representative point along part I of the map, whereas turbulent phases are associated with transitions of the representative point to the part labeled II and back again. It has been shown $[54,55]$ by using a renormalization T-group technique that the mean duration $\tau$ of laminar phases is proportional to $\varepsilon^{-(1-1 / q)}$. The same result is obtained in $[53,35,52]$ by replacing the difference equation (2.49) by the corresponding differential equation. In [53-55,35,52] the influence of external noise is also considered. It is found that, for $\varepsilon=0, \tau \sim g^{-2(q-1) /(q+1)}$.

The presence of external noise can be described by an additional term in Eq. (2.49), namely

$$
x_{n+1}=\varepsilon+x_{n}+a x_{n}^{q}+g \xi_{n},
$$

where $\xi_{n}$ is white noise with zero mean. We assume that $\left\langle\xi_{n} \xi_{m}\right\rangle=\delta_{n m}$, where $\delta_{n m}$ is the Kronecker delta. For sufficiently small $\varepsilon, g$ and $a\left(x^{(1),(2)}\right)^{q}$ Eq. (2.50) can be replaced by the following differential equation $[53,35]$ :

$$
\dot{x}=\varepsilon+a x^{q}+g \xi(t),
$$

where $\langle\xi(t)\rangle=0,\left\langle\xi(t) \xi\left(t^{\prime}\right)\right\rangle=\delta\left(t-t^{\prime}\right)$. The Fokker-Planck equation for the probability density $w(x, t)$ associated with Eq. (2.51) for $x \leq x^{(2)}$ is

$$
\frac{\partial w}{\partial t}=-\frac{\partial}{\partial x}\left(\left(\varepsilon+a x^{q}\right) w\right)+\frac{g^{2}}{2} \frac{\partial^{2} w}{\partial x^{2}} .
$$

To calculate the mean duration of laminar phases it is sufficient to find a steady-state solution of Eq. (2.52) satisfying the normalization condition and the zero boundary condition at the point $x=x^{(2)}{ }^{6}$ Because all points attaining the boundary $x^{(2)}$ leave the interval in question, we put

$$
w\left(x^{(2)}\right)=0 .
$$

For $x^{(1)} \leq x \leq x^{(2)}$ the steady-state solution of Eq. (2.52) with the boundary condition (2.53) is

$$
w(x)=\frac{2 G_{0}}{g^{2}} \exp \left[\frac{2}{g^{2}}\left(\varepsilon x+\frac{a x^{q+1}}{q+1}\right)\right] \int_{x}^{x^{(2)}} \exp \left[-\frac{2}{g^{2}}\left(\varepsilon y+\frac{a y^{q+1}}{q+1}\right)\right] \mathrm{d} y,
$$

where $G_{0}$ is the value of the probability flux

$$
G=\left(\varepsilon+a x^{q}\right) w-\frac{g^{2}}{2} \frac{\mathrm{d} w}{\mathrm{~d} x}
$$

\footnotetext{
${ }^{5}$ The sense of $x^{(1)}$ and $x^{(2)}$ is clear from Fig. 1.

${ }^{6}$ The authors of [53] set an unjustified second boundary condition $w\left(x^{(1)}\right)=0$ and added a constant source in Eq. (2.52).
} 
within the interval $x^{(1)} \leq x \leq x^{(2)}$. For $x<x^{(1)}$ the probability flux is equal to zero, and therefore

$$
w(x)=\frac{2 G_{0}}{g^{2}} \exp \left[\frac{2}{g^{2}}\left(\varepsilon x+\frac{a x^{q+1}}{q+1}\right)\right] \int_{x^{(1)}}^{x^{(2)}} \exp \left[-\frac{2}{g^{2}}\left(\varepsilon y+\frac{a y^{q+1}}{q+1}\right)\right] \mathrm{d} y .
$$

The value of the probability flux $G_{0}$ is determined from the normalization condition by integrating (2.54) and(2.55) with respect to $x$ from $-\infty$ to $x^{(2)}$. It follows from the general theory of fluctuational transitions set forth above that the mean duration of laminar phases $\tau$ is equal to $G_{0}^{-1}$. Thus,

$$
\begin{aligned}
\tau= & \frac{2}{g^{2}}\left(\int_{x^{(1)}}^{x^{(2)}} \exp \left[\frac{2}{g^{2}}\left(\varepsilon x+\frac{a x^{q+1}}{q+1}\right)\right] \int_{x}^{x^{(2)}} \exp \left[-\frac{2}{g^{2}}\left(\varepsilon y+\frac{a y^{q+1}}{q+1}\right)\right] \mathrm{d} y \mathrm{~d} x\right. \\
& \left.+\int_{-\infty}^{x^{(1)}} \exp \left[\frac{2}{g^{2}}\left(\varepsilon x+\frac{a x^{q+1}}{q+1}\right)\right] \mathrm{d} x \int_{x^{(1)}}^{x^{(2)}} \exp \left[-\frac{2}{g^{2}}\left(\varepsilon y+\frac{a y^{q+1}}{q+1}\right)\right] \mathrm{d} y\right) .
\end{aligned}
$$

In the simplest case when $g \equiv 0$, i.e., in the absence of external noise, it immediately follows from Eq. (2.52) that

$$
w(x)= \begin{cases}\frac{G_{0}}{\varepsilon+a x^{q}} & \text { for } x^{(1)} \leq x \leq x^{(2)}, \\ 0 & \text { for } x<x^{(1)} .\end{cases}
$$

It can be seen from (2.57) that in the specific case when $q=2$ the probability distribution takes the form of a Lorentzian with its maximum at $x=0$; the width of this line is equal to $\sqrt{\varepsilon / a}$. The value of $G_{0}$ in (2.57) can be calculated explicitly for sufficiently small $\varepsilon$ when we can put $x^{(1)} \approx-\infty$ and $x^{(2)} \approx \infty$. In this case we find

$$
\tau \approx \frac{2 \pi}{q \sin (\pi / q)}\left(a \varepsilon^{q-1}\right)^{-1 / q} .
$$

For $q=2$ we have $\tau \sim \varepsilon^{-1 / 2}$. In another specific case when $\varepsilon=0, g \neq 0$ we obtain from (2.56):

$$
\tau=2^{(q-1) /(q+1)}\left(\frac{q+1}{a}\right)^{2 /(q+1)} B g^{-2(q-1) /(q+1)},
$$

where $B$ is determined by the formula

$$
\begin{aligned}
& B=\int_{u_{1}}^{u_{2}} \exp \left(u^{q+1}\right) \int_{u}^{u_{2}} \exp \left(-v^{q+1}\right) \mathrm{d} v \mathrm{~d} u+\int_{-\infty}^{u_{1}} \exp \left(u^{q+1}\right) \mathrm{d} u \int_{u_{1}}^{u_{2}} \exp \left(-v^{q+1}\right) \mathrm{d} v, \\
& u_{1,2}=\left(\frac{2 a}{(q+1) g^{2}}\right)^{1 /(q+1)} x^{(1),(2)} .
\end{aligned}
$$

For sufficiently small external noise, when $g^{2} \ll a\left|x^{(1),(2)}\right|^{z+1}$ we can put $u_{1} \approx-\infty$ and $u_{2} \approx \infty$. In this case $B$ is independent of $g$ and we have from (2.59) $\tau \sim g^{-2(q-1) /(q+1)}$. In particular, for $q=2$, $\tau \sim g^{-2 / 3}$.

\subsubsection{On-off intermittency}

Recently another type of intermittency was discovered. It is known as on-off intermittency. This term was introduced after the work [56], though a map associated with the similar type of 
intermittent behavior was first considered by Pikovsky [57] and then by Fujisaka and Yamada [58]. An important point is that this type of intermittency can occur not only in coupled dynamical systems but in stochastic systems as well [59]. In [59] the statistical properties of on-off intermittency were studied through analysis of the map

$$
x_{n+1}=a\left(1+y_{n}\right) x_{n}+f\left(x_{n}\right),
$$

where $y_{n}$ is either a deterministic chaotic process or a random process, $a$ is the bifurcation parameter, and $f\left(x_{n}\right)$ is a nonlinear function free from a linear term. For this map it was shown that for $a>0$ the mean duration of laminar phases is proportional to $a^{-1}$. The influence of weak additive noise on the characteristics of on-off intermittency was considered by Chenis and Lustfeld [60].

We consider on-off intermittency by reference to a specific example system described by the following model equations:

$$
\dot{x}=-\left(b+\alpha r^{2}\right) x+x\left(\xi_{1}(t)+\frac{\xi_{2}(t)}{r}\right), \quad \dot{y}=-\left(b+\alpha r^{2}\right) y+y\left(\xi_{1}(t)+\frac{\xi_{2}(t)}{r}\right),
$$

where $r=\sqrt{x^{2}+y^{2}}$, and $\xi_{1}(t)$ and $\xi_{2}(t)$ are white noises of intensities $\kappa_{1}$ and $\kappa_{2}$, respectively. Eqs. (2.61) can be rewritten in polar coordinates $r$ and $\varphi=\arctan (y / x)$ as

$$
\dot{r}=-\left(b+\alpha r^{2}\right) r+r \xi_{1}(t)+\xi_{2}(t), \quad \dot{\varphi}=0 .
$$

We see that $\xi_{1}(t)$ and $\xi_{2}(t)$ represent multiplicative and additive noise respectively. The first of Eqs. (2.62) is of the same form as (2.7). Hence, the Fokker-Planck equation associated with this equation can be written as

$$
\frac{\partial w}{\partial t}=-\frac{\partial}{\partial r}\left(\left(b a-\alpha r^{2}\right) r w(r, \varphi, t)\right)+\frac{1}{2} \frac{\partial^{2}}{\partial r^{2}}\left(\left(r^{2} \kappa_{1}+\kappa_{2}\right) w(r, \varphi, t)\right),
$$

where $a=\kappa_{1} / 2 b-1$ is the bifurcation parameter. To calculate the mean duration of the laminar phases, we assume that our system is in a laminar phase if $r \leq \varepsilon$, where $\varepsilon$ is a given small quantity. The mean duration $\tau_{\varepsilon}$ of the laminar phases is determined by the mean duration of a random walk-like motion of a representative point on the phase plane $x y$ inside a circle of radius $\varepsilon$. As shown above, this duration can be calculated by using the steady-state solution of Eq. (2.63) with the boundary condition

$$
\left.w(r, \varphi)\right|_{r=\varepsilon}=0 .
$$

This solution is

$$
\begin{aligned}
w(r, \varphi)= & \frac{2 G}{\kappa_{1}}\left(r^{2}+\frac{\mu}{2(a+1)}\right)^{\left(\alpha \mu+\kappa_{1} a\right) / 2(a+1) \kappa_{1}-1} \exp \left(-\frac{\alpha r^{2}}{\kappa_{1}}\right) \\
& \times \int_{r}^{\varepsilon}\left(\rho^{2}+\frac{\mu}{2(a+1)}\right)^{-\left(\alpha \mu+\kappa_{1} a\right) / 2(a+1) \kappa_{1}} \exp \left(\frac{\alpha \rho^{2}}{\kappa_{1}}\right) \mathrm{d} \rho,
\end{aligned}
$$

where $G$ is the probability flux across unit of length of any circumference inside the circle of radius $\varepsilon, \mu=\kappa_{2} / b$. The value of $G$ is determined from the normalization condition by integrating 
expression (2.65) over the circle of radius $\varepsilon$ :

$$
\begin{aligned}
G^{-1}= & \frac{4 \pi}{\kappa_{1}} \int_{0}^{\varepsilon} r\left(r^{2}+\frac{\mu}{2(a+1)}\right)^{\left(\alpha \mu+\kappa_{1} a\right) / 2(a+1) \kappa_{1}-1} \exp \left(-\frac{\alpha r^{2}}{\kappa_{1}}\right) \\
& \times \int_{r}^{\varepsilon}\left(\rho^{2}+\frac{\mu}{2(a+1)}\right)^{-\left(\alpha \mu+\kappa_{1} a\right) / 2(a+1) \kappa_{1}} \exp \left(\frac{\alpha \rho^{2}}{\kappa_{1}}\right) \mathrm{d} \rho \mathrm{d} r .
\end{aligned}
$$

As already noted, the mean time at which the representative point first attains the circle boundary $r=\varepsilon$ is determined by total probability flux across this boundary, i.e., $T=(2 \pi \varepsilon G)^{-1}$. Taking account of the fact that the representative point touching the boundary can return again with probability $(1-p)$, we obtain for the mean duration $\tau$ of the laminar phases the following expression $[38,39]$ :

$$
\tau=T p \sum_{j=1}^{\infty} j(1-p)^{j-1} .
$$

Summing the series we have

$$
\tau=T / p \text {. }
$$

Let us consider the specific case when $\alpha \varepsilon^{2} / \kappa_{1} \ll 1$. We can then ignore the terms $\exp \left(-\alpha r^{2} / \kappa_{1}\right)$ and $\exp \left(\alpha \rho^{2} / \kappa_{1}\right)$. As a result we obtain

$$
T=\frac{2}{\kappa_{1} \varepsilon} \int_{0}^{\varepsilon} r\left(r^{2}+\frac{\mu}{2(a+1)}\right)^{\left(\alpha \mu+\kappa_{1} a\right) / 2(a+1) \kappa_{1}-1} \int_{r}^{\varepsilon}\left(\rho^{2}+\frac{\mu}{2(a+1)}\right)^{-\left(\alpha \mu+\kappa_{1} a\right) / 2(a+1) \kappa_{1}} \mathrm{~d} \rho \mathrm{d} r .
$$

To calculate the inner integral approximately, we multiply and divide the integrand by $\rho$, substitute $y$ for $\rho^{2}$ and take $\varepsilon / 2$ in place of $\rho$. This manipulation gives

$$
\begin{aligned}
T= & \frac{2(a+1)}{\kappa_{1}(a+2)-\alpha \mu}\left\{\frac{2 \kappa_{1}(a+1)}{\kappa_{1} a+\alpha \mu}\left(1+\frac{\mu}{2(a+1) \varepsilon^{2}}\right)\right. \\
& \left.\times\left[1-\left(\frac{\mu}{2(a+1) \varepsilon^{2}+\mu}\right)^{\left(\kappa_{1} a+\alpha \mu\right) / 2(a+1) \kappa_{1}}\right]-1\right\} .
\end{aligned}
$$

In particular, for $\mu=0$ (additive noise is absent) we obtain from (2.69) the following simple formula:

$$
T=1 / b a .
$$

So, for $\mu=0$, we have the same dependence of $\tau$ on $a$ as for the map (2.60); whereas, for $\mu \neq 0$, the dependence is more complicated. For $\mu b \ll \varepsilon^{2} \kappa_{1}$ we have $T=2(a+1) /\left(\kappa_{1} a+\alpha \mu\right)$, i.e., nonlinearity plays an important role in this dependence, given that the intermittent behavior begins to show itself for $a<a_{\mathrm{cr}}=0$. In another limiting case, when $\mu b \gg \varepsilon^{2} \kappa_{1}, T=\varepsilon^{2} / 2 \kappa_{2}$, i.e., for very small $\varepsilon$ or $\kappa_{1}$ the mean duration of laminar phases is independent of $a$. For $a=0, \alpha \mu \ll b$ we obtain from (2.70) the following dependence of $T$ on the intensity of additive noise, conveniently characterized by the parameter $\kappa=\mu / 2 \varepsilon^{2}$ :

$$
T=\frac{1}{b}\left((1+\kappa) \ln \frac{1+\kappa}{\kappa}-1\right),
$$


i.e., the decline of the mean duration of laminar phases with increasing intensity of additive noise is logarithmic in character. The dependences of $b T$ on $a$ for different values of $\kappa_{2}$ constructed by the formula (2.69) are shown in Fig. 2 for two cases: (a) when the nonlinearity characterized by the parameter $\beta=\alpha \varepsilon^{2} / b$ can be neglected, and (b) when it is taken into account. We see that the nonlinearity exerts only a small influence on these dependences. The dependences of $b T$ on $\kappa_{2}$ for different values of $a$ are given in Fig. 3 for the same two cases.
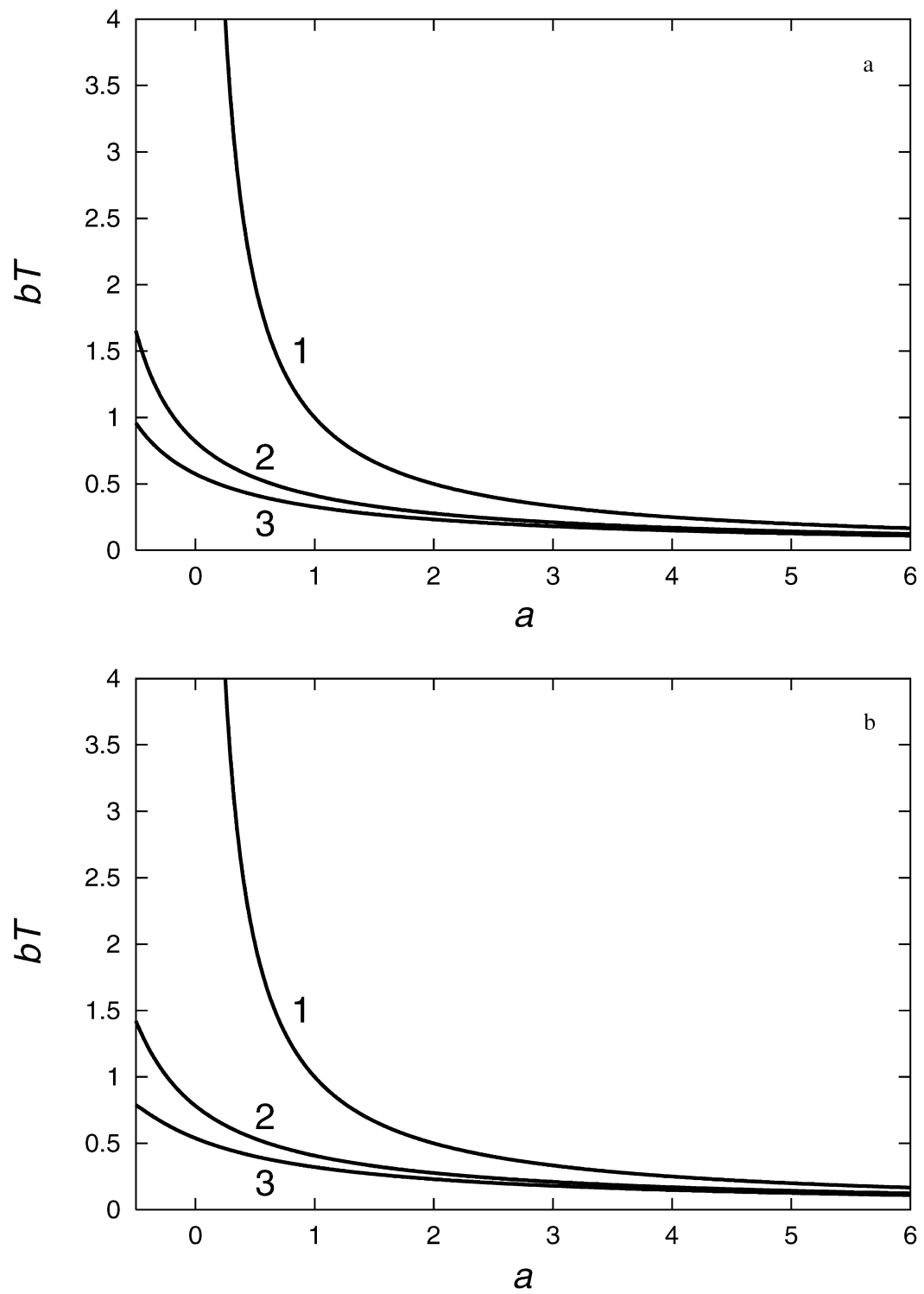

Fig. 2. Dependence of $b T$ on $a$ in the case where $\alpha \varepsilon^{2} / \kappa_{1} \ll 1$ for $\kappa_{2}=0$ (curves labelled 1), $\kappa_{2}=0.1$ (curves labelled 2), $\kappa=0.2$ (the curves labelled 3), for: (a) $\beta=0$; and (b) $\beta=1$. 

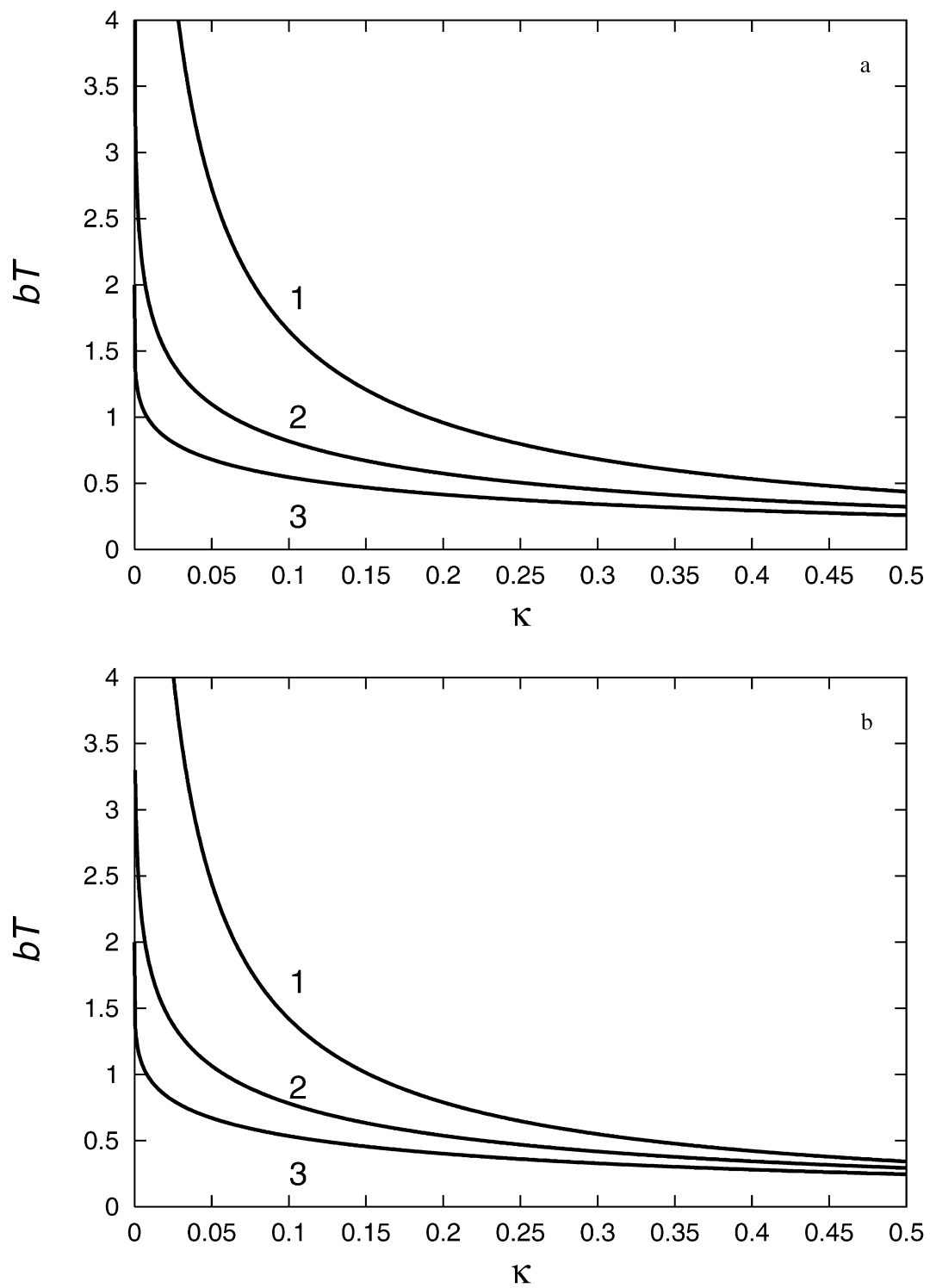

Fig. 3. Dependence of $b T$ on $\kappa_{2}$ in the case where $\alpha \varepsilon^{2} / \kappa_{1} \ll 1$ for $a=-0.5$ (curves labelled 1), $a=0$ (curves labelled 2), $a=0.5$ (curves labelled 3), for: (a) $\beta=0$; and (b) $\beta=1$.

\section{Noise-induced transport of Brownian particles (stochastic ratchets)}

In recent years fluctuation-induced transport phenomena for Brownian particles have attracted considerable interest, usually in the context of biological and chemical problems (see, for example, [61-66,20,22,24]). A physical experiment demonstrating the possibility of such transport in a ratchet-like potential field created by laser beam is described in [67]. In [68] it was experimentally shown that directed motion of a particle can be induced merely by turning on and off 
a periodic asymmetric potential (more recently, this phenomenon become known as a flashing ratchet). Similar experiments are also presented in [69].

Systems in which noise-induced transport occurs are often called stochastic ratchet-like devices by analogy with mechanical device "ratchet and pawl" described and considered by Feynman [70]. Feynman showed that in the case of thermodynamic equilibrium the ratchet on average is at rest - it advances and retreats by an equal number of teeth on the wheel - as it must be because of the Second Law of Thermodynamics. It is interesting that similar considerations were discussed by Smoluchowski [71] well before Feynman. The "ratchet and pawl" device constitutes a mechanical rectifier. It is similar in essence to an electrical rectifier. However, as is often the case, the problems associated with electrical rectification of fluctuations were discussed independently of the ratchet problems [72-77]. In [73,74] it was found that in the simplest electrical rectifier, consisting of capacitor and diode, the capacitor can be charged without an external source, at the expense of only thermal fluctuations. This paradoxical result cast some doubt on the applicability of the Second Law of Thermodynamics to the phenomenon considered [76]. As far back as 1950, however, considering the diode as a nonlinear resistor, Brillouin [72] showed that, for the Second Law to apply, a shift of the characteristic of the nonlinear resistor must be taken into account. Stratonovich [77,78] established, on a certain model of diode, that such a shift does indeed occur due to fluctuations of the current through the diode considered as a nonlinear resistor, and he calculated it. With this shift, the mean value of the voltage drop across the capacitor is found to vanish for the case of thermodynamical equilibrium.

Most commonly, consideration of noise-induced transport is restricted to the so-called overdamped case, when the mass of the Brownian particle can be neglected and its motion is described by a first order differential equation of the form

$$
\dot{x}+f(x)=\varphi(t)+\zeta(x, t)+\xi(t),
$$

where $f(x)$ is a periodic function of $x$ possessing an asymmetry, $\varphi(t)$ is a regular periodic force, $\zeta(x, t)$ is a random process with zero mean value, and $\xi(t)$ is white noise of intensity $K$ imitating thermal fluctuations. The process $\zeta(x, t)$ can be either given or described by additional equations.

More often than not researchers of noise-induced transport set the function $f(x)$ to a form corresponding to a saw-tooth potential $U(x)=\int_{0}^{x} f(x) \mathrm{d} x$ shown in Fig. 4. In this case

$$
f(x)=\left\{\begin{array}{cc}
a_{1} & \text { for } n L<x<n L+x_{1}, \\
-a_{2} & \text { for } n L-x_{2}<x<n L,
\end{array}\right.
$$

where $n=0, \pm 1, \pm 2, \ldots, L=x_{1}+x_{2}$ is the period of the function $f(x)$. It is easily shown that, in the absence of the disturbances $\zeta(x, t)$ and $\xi(t)$, the points $x=n L$ and $x=n L+x_{1}=(n+1) L-x_{2}$ correspond to stable and unstable equilibrium states, respectively. If there are fluctuations then transitions from one stable state to another can occur. Directional motion of the particle will occur if the probabilities of transitions in opposite directions are different. So, we see that the problem of noise-induced transport is closely allied to the fluctuational transitions considered above.

It is usual to distinguish two types of ratchet devices $[64,79,65,20,80]$ : (a) where $\zeta(x, t)$ is a force independent of $x$; and (b) where $\zeta(x, t)$ depends on $x$. In its turn, the latter can be also divided into two subclasses: (i) those where $\zeta(x, t)=f(x) \chi(t)$, which is to say that the height of the potential barrier fluctuates [64]; and (ii) where $\zeta(x, t)$ is a random function of $t$ and $x$ [80]. 


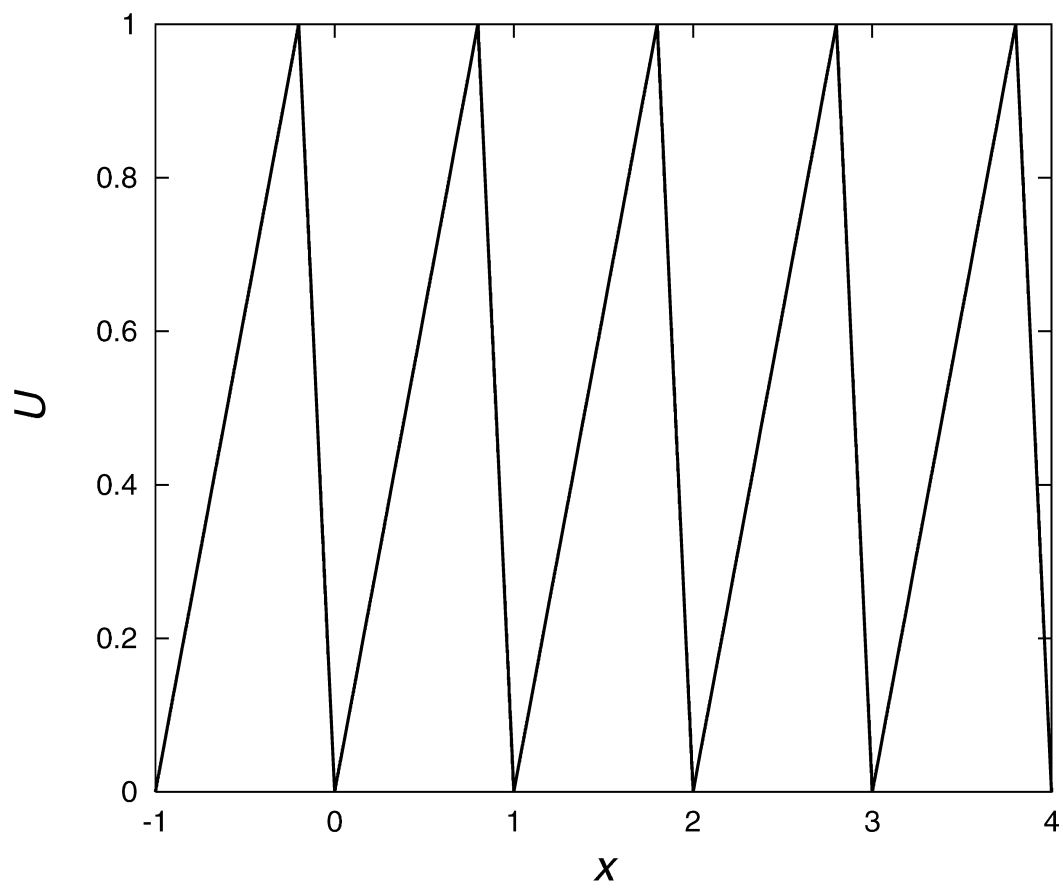

Fig. 4. An example of a saw-tooth potential $U(x)$.

In the last few years much attention has been concentrated on the possibility of exploiting such phenomena to separate particles of different mass or size. In this connection studies of different models giving flux reversals as the system parameters change are very important $[81-83,85,86]$. Flux reversals induced by noise colour alone, predicted in [82], were subsequently observed in analogue electronic experiments [84].

We consider below the one-dimensional motion of a Brownian particle in a viscous medium described by the following equation:

$$
\mu \ddot{x}+\dot{x}+f(x)=\varphi(t)+\zeta(x, t)+\xi(t),
$$

where $\mu=m / \beta, m$ is the particle mass, $\beta$ is the viscous friction factor, $f(x)$ is described by expression (3.2), and $\varphi(t), \zeta(x, t)$ and $\xi(t)$ are the same that in Eq. (3.1). For $\mu=0$ this equation reduces to Eq. (3.1).

\subsection{Noise-induced transport of light Brownian particles in a viscous medium} with a saw-tooth potential

Here we consider the case when viscous friction in the medium is sufficiently large and mass of the particle is sufficiently small, that the motion of the particle can be described approximately by Eq. (3.1). In addition, we assume that $\zeta(x, t)$ is white noise of intensity $f^{2}(x) K_{1}$ which is uncorrelated 
with $\xi(t)$. The Fokker-Planck equation associated with Eq. (3.1) at the specified conditions is

$$
\frac{\partial w}{\partial t}=-\frac{\partial}{\partial x}\left(\left(\varphi(t)-f(x)+\frac{K_{1}}{2} f(x) f^{\prime}(x)\right) w(x, t)\right)+\frac{1}{2} \frac{\partial^{2}}{\partial x^{2}}(K(x) w(x, t)),
$$

where $K(x)=K+f^{2}(x) K_{1}$. Because $f(x)$ is a periodic function of $x, w(x, t)$ is also a periodic function of $x$. Thus Eq. (3.4) only needs to be solved within the interval from $-x_{2}$ to $x_{1}$.

Let us show that the statistical average of the particle velocity $\dot{x}$ is determined by the relationship

$$
\langle\dot{x}\rangle=\int_{-x_{2}}^{x_{1}} G(x, t) \mathrm{d} x,
$$

where

$$
G(x, t)=-\frac{1}{2} \frac{\partial(K(x) w(x, t))}{\partial x}+F(x, t) w(x, t)
$$

can be treated as the instantaneous probability flux, $F(x, t)=\varphi(t)-f(x)+\left(K_{1} / 2\right) f(x) f^{\prime}(x)$. Averaging Eq. (3.1) over statistical ensemble and taking into account that the random process $\xi(t)$ has zero mean value and $\langle\zeta(x, t)\rangle=\left(K_{1} / 2\right) f(x) f^{\prime}(x)$, we obtain

$$
\langle\dot{x}\rangle=\langle F(x, t)\rangle=\int_{-x_{2}}^{x_{1}} F(x, t) w(x, t) \mathrm{d} x .
$$

According to (3.6), this expression can be rewritten as

$$
\langle\dot{x}\rangle=\int_{-x_{2}}^{x_{1}} F(x, t) w(x, t) \mathrm{d} x=\int_{-x_{2}}^{x_{1}}\left(G(x, t)+\frac{1}{2} \frac{\partial(K(x) w(x, t))}{\partial x}\right) \mathrm{d} x .
$$

Because of the spatial periodicity of the functions $w(x, t)$ and $K(x)$ we obtain the formula (3.5). Averaging (3.5) over time we have

$$
\overline{\langle\dot{x}\rangle}=\int_{-x_{2}}^{x_{1}} \overline{G(x, t)} \mathrm{d} x,
$$

where

$$
\overline{G(x, t)}=\lim _{T \rightarrow \infty} \frac{1}{T} \int_{0}^{T} G(x, t) \mathrm{d} t .
$$

So, the mean particle velocity, i.e., its net diffusive drift, is proportional to the probability flux averaged over both space and over time.

\subsubsection{The case of an additional regular force}

We now consider the case when $K_{1}=0$ and $\varphi(t) \neq 0$. If the function $\varphi(t)$ is sufficiently slow, we can use the successive approximation technique for solving Eq. (3.4) by putting

$$
w(x, t)=w_{0}(x, \varphi)+w_{1}(x, \varphi) \dot{\varphi}+w_{21}(x, \varphi) \ddot{\varphi}+w_{22}(x, \varphi) \dot{\varphi}^{2}+\cdots .
$$


As a zeroth-order approximation, we take a quasistationary solution of Eq. (3.4) for which $\partial w / \partial t=0$. In this approximation we obtain the following equation for $w_{0}(x, \varphi)$ :

$$
\frac{K}{2} \frac{\partial w_{0}(x, \varphi)}{\partial x}-(\varphi-f(x)) w_{0}(x, \varphi)=-G_{0}(\varphi),
$$

where $G_{0}(\varphi)$ is the probability flux in the zero approximation. Solving Eq. (3.9) with account taken of (3.2) we find

where

$$
w_{0}(x, \varphi)=\left\{\begin{array}{l}
\left(C_{0}(\varphi)-\frac{G_{0}(\varphi)}{q_{1}}\right) \exp \left(\frac{2 q_{1} x}{K}\right)+\frac{G_{0}(\varphi)}{q_{1}} \text { for } 0 \leq x \leq x_{1}, \\
\left(C_{0}(\varphi)-\frac{G_{0}(\varphi)}{q_{2}}\right) \exp \left(\frac{2 q_{2} x}{K}\right)+\frac{G_{0}(\varphi)}{q_{2}} \text { for }-x_{2} \leq x \leq 0,
\end{array}\right.
$$

$$
q_{1,2}=\varphi \mp a_{1,2},
$$

and $C_{0}(\varphi)$ is an arbitrary function of $\varphi$. From the periodicity condition of the function $w_{0}(x, \varphi)$ we find the relation between $G_{0}(\varphi)$ and $C(\varphi): C_{0}(\varphi)=Q(\varphi) G_{0}(\varphi)$, where

$$
Q(\varphi)=\frac{1}{q_{1} q_{2}} \frac{q_{2} \exp \left(2 U_{0} \varphi / K a_{1}\right)-q_{1} \exp \left(-2 U_{0} \varphi / K a_{2}\right)-\left(q_{2}-q_{1}\right) \exp \left(2 U_{0} / K\right)}{\exp \left(2 U_{0} \varphi / K a_{1}\right)-\exp \left(-2 U_{0} \varphi / K a_{2}\right)},
$$

and $U_{0}=a_{1} x_{1}=a_{2} x_{2}$ is the height of the potential barrier. The probability flux $G_{0}(\varphi)$ can be found from the normalization condition for the probability density $w_{0}(x, \varphi)$. In so doing we obtain

$$
\begin{aligned}
G_{0}^{-1}(\varphi)= & U_{0}\left(\frac{1}{a_{1} q_{1}}+\frac{1}{a_{2} q_{2}}\right)+\frac{K\left(a_{1}+a_{2}\right)^{2}}{2 q_{1}^{2} q_{2}^{2}} \exp \left(-\frac{2 U_{0}}{K}\right) \\
& \times \frac{\left(\exp \left(2 U_{0} \varphi / K a_{1}\right)-\exp \left(2 U_{0} / K\right)\right)\left(\exp \left(-2 U_{0} \varphi / K a_{2}\right)-\exp \left(2 U_{0} / K\right)\right)}{\exp \left(2 U_{0} \varphi / K a_{1}\right)-\exp \left(-2 U_{0} \varphi / K a_{2}\right)} .
\end{aligned}
$$

In the most interesting case when $\varphi$ is sufficiently small, namely when

$$
\max \varphi \ll \frac{a_{1} a_{2}}{a_{2}-a_{1}} \min \left(1, \frac{K}{U_{0}}\right),
$$

we find

$$
G_{0}(\varphi)=G_{00} \varphi+G_{01} \varphi^{2}+\cdots,
$$

where

$$
\begin{aligned}
G_{00} & =\frac{U_{0} a_{1} a_{2}}{K^{2}\left(a_{1}+a_{2}\right) \sinh ^{2}\left(U_{0} / K\right)}, \\
G_{01} & =G_{00} \frac{a_{2}-a_{1}}{a_{1} a_{2}}\left(\frac{U_{0}^{2}}{K^{2} \sinh ^{2}\left(U_{0} / K\right)}+\frac{U_{0}}{K \tanh \left(U_{0} / K\right)}-2\right) .
\end{aligned}
$$

For simplicity, we further restrict ourselves to the case when condition (3.14) is valid. In this case, we have from (3.12), (3.11), (3.15) and (3.8)

$$
C_{0}(\varphi)=C_{00}+C_{01} \varphi+\cdots,
$$


where

$$
\begin{aligned}
& C_{00}=\frac{2 a_{1} a_{2}}{K\left(a_{1}+a_{2}\right)\left(1-\mathrm{e}^{-2 U_{0} / K}\right)}, \quad C_{01}=\frac{a_{2}-a_{1}}{a_{1} a_{2}} C_{00}\left(\frac{U_{0}^{2}}{K^{2} \sinh ^{2}\left(U_{0} / K\right)}-1\right), \\
& w(x, t)=w_{0}(x, \varphi)+\left(w_{10}(x)+w_{11}(x) \varphi\right) \dot{\varphi}+\left(w_{20}(x)+w_{21}(x) \varphi\right) \ddot{\varphi}+w_{22}(x) \dot{\varphi}^{2}+\cdots .
\end{aligned}
$$

It follows from (3.8) and (3.18) that the functions $w_{10}(x), w_{11}(x), w_{20}(x), w_{21}(x)$, and $w_{22}(x)$ are described by the equations

$$
\begin{aligned}
& \frac{K}{2} \frac{\mathrm{d} w_{10}(x)}{\mathrm{d} x}+f(x) w_{10}(x)=-G_{10}(x), \quad G_{10}(x)=-\left.\int_{0}^{x} \frac{\partial w_{0}(x, \varphi)}{\partial \varphi}\right|_{\varphi=0} \mathrm{~d} x+G_{10}, \\
& \frac{K}{2} \frac{\mathrm{d} w_{11}(x)}{\mathrm{d} x}+f(x) w_{11}(x)=-G_{11}(x), \quad G_{11}(x)=-\left.\int_{0}^{x} \frac{\partial^{2} w_{0}}{\partial \varphi^{2}}\right|_{\varphi=0} \mathrm{~d} x-w_{10}(x)+G_{11}, \\
& \frac{K}{2} \frac{\mathrm{d} w_{20}(x)}{\mathrm{d} x}+f(x) w_{20}(x)=-G_{20}(x), \quad G_{20}(x)=-\int_{0}^{x} w_{10}(x) \mathrm{d} x+G_{20}, \\
& \frac{K}{2} \frac{\mathrm{d} w_{21}(x)}{\mathrm{d} x}+f(x) w_{21}(x)=-G_{21}(x), \quad G_{21}(x)=-\int_{0}^{x} w_{11}(x) \mathrm{d} x-w_{20}(x)+G_{21}, \\
& \frac{K}{2} \frac{\mathrm{d} w_{22}(x)}{\mathrm{d} x}+f(x) w_{22}(x)=-G_{22}(x), \quad G_{22}(x)=-\int_{0}^{x} w_{10}(x) \mathrm{d} x+G_{22} .
\end{aligned}
$$

So, for small $\varphi$ we find

$$
\overline{\langle\dot{x}\rangle} \approx \overline{G_{0}} L+\frac{B^{2} \omega^{2}}{2} \int_{-x_{2}}^{x_{1}}\left(G_{22}(x)-G_{21}(x)\right) \mathrm{d} x=\left(\overline{G_{0}}+G_{2} \frac{B^{2} \omega^{2}}{2}\right) L,
$$

where $G_{2}=G_{22}-G_{21} \cdot{ }^{7}$ Thus, the correction to the quasistationary solution is proportional to the frequency squared and to the value of $G_{2}$. Subtracting (3.22) from (3.23) we obtain the equation for $W(x) \equiv w_{22}(x)-w_{21}(x)$ :

$$
\frac{K}{2} \frac{\mathrm{d} W(x)}{\mathrm{d} x}+f(x) W(x)=-\left(w_{20}(x)+G_{2}\right) .
$$

A solution of this equation is

$$
W(x)=\left\{\begin{array}{l}
\left(C+\frac{G_{2}}{a_{1}}-\frac{2}{K} \int_{0}^{x} w_{20}\left(x^{\prime}\right) \exp \left(\frac{2 a_{1} x^{\prime}}{K}\right) \mathrm{d} x^{\prime}\right) \exp \left(-\frac{2 a_{1} x}{K}\right)-\frac{G_{2}}{a_{1}} \text { for } 0 \leq x \leq x_{1}, \\
\left(C-\frac{G_{2}}{a_{2}}-\frac{2}{K} \int_{0}^{x} w_{20}\left(x^{\prime}\right) \exp \left(-\frac{2 a_{2} x^{\prime}}{K}\right) \mathrm{d} x^{\prime}\right) \exp \left(\frac{2 a_{2} x}{K}\right)+\frac{G_{2}}{a_{2}} \text { for }-x_{2} \leq x \leq 0 .
\end{array}\right.
$$

\footnotetext{
${ }^{7}$ Here we have taken into account that, as follows from the normalization condition for the function $w(x, t)$, the integrals of $w_{1}(x, \varphi), w_{21}(x, \varphi)$ and $w_{22}(x, \varphi)$ with respect to $x$ between the limits $x=-x_{2}$ and $x=x_{1}$ must be equal to zero.
} 
From the periodicity condition we find $G_{2}$ :

$$
G_{2}=-\frac{2 a_{1} a_{2}}{K\left(a_{1}+a_{2}\right)\left(\mathrm{e}^{2 U_{0} / K}-1\right)} \int_{-x_{2}}^{x_{1}} w_{20}(x) \exp \left(\frac{2 f(x) x}{K}\right) \mathrm{d} x .
$$

So, to calculate $G_{2}$ we must find $w_{20}(x)$ which in turn is determined by Eq. (3.21). To solve this equation we must previously have solved Eq. (3.19). Although this procedure is simple in principle, it leads to rather cumbersome expressions. Therefore we restrict ourselves to the analysis of the zeroth-order approximation. If condition (3.14) is fulfilled and $\varphi(t) \equiv B_{0}=$ const (in addition to $f(x)$ a constant force acts upon the particle), then

$$
\overline{\langle\dot{x}\rangle} \approx \frac{U_{0}^{2}}{K^{2} \sinh ^{2}\left(U_{0} / K\right)} B_{0},
$$

i.e., the particle moves in the direction of this constant force no matter what the relation between $a_{1}$ and $a_{2}$. We see from (3.28) that in the absence of fluctuations, when $K \rightarrow 0, \overline{\langle\dot{x}\rangle} \rightarrow 0$, i.e., transport of the particle is impossible despite the presence of the constant force. This is because the force is small and cannot by itself push the particle over the potential barrier. In another specific case, when $\varphi(t)=B \cos \omega t$, where $B$ is sufficiently small, we obtain

$$
\overline{\langle\dot{x}\rangle} \approx \frac{U_{0}^{2}\left(a_{2}-a_{1}\right) B^{2}}{2 K^{2} a_{1} a_{2} \sinh ^{2}\left(U_{0} / K\right)}\left(\frac{U_{0}^{2}}{K^{2} \sinh ^{2}\left(U_{0} / K\right)}+\frac{U_{0}}{K \tanh \left(U_{0} / K\right)}-2\right),
$$

i.e., the particle moves in the direction of the slower rate of potential change. It is easy to verify that, in the absence of fluctuations, transport of the particle cannot occur, just as in the case of a small constant force.

Examples of the dependences of $\overline{\langle\dot{x}\rangle} / B^{2}$ on $K / U_{0}$ described by formulas (3.7), (3.13) are shown in Fig. 5 for a number of values of $B$. We see that these dependences are of radically different kinds for $B<\min \left(a_{1}, a_{2}\right)$ and $B>\min \left(a_{1}, a_{2}\right)$. In the first case these dependences have a maximum at a certain value of $K / U_{0}$ that is the smaller the greater is $B$. For $K / U_{0} \rightarrow 0$, i.e., in the absence of the thermal fluctuations, $\overline{\langle\dot{x}\rangle} / B^{2} \rightarrow 0$. In the second case $\overline{\langle\dot{x}\rangle} / B^{2}$ tends to a certain finite value as $K / U_{0} \rightarrow 0$, which can be calculated from the theory of vibrational transport $[87,86]$. For $B<0.5$ the dependences found are almost coincident with those described by the approximate formula (3.29). In this case the averaged particle velocity is maximal for $K / U_{0} \approx 0.43$. In the case that $B<\min \left(a_{1}, a_{2}\right)$, the ratio $K / U_{0}$ is either very small or very large, noise-induced transport is not feasible.

The results obtained can be explained in the following manner. Noise-induced transport can occur if fluctuational transitions through each potential barrier are more frequent in one direction than in another. Because the probability of the transition through a certain potential barrier depends only on its height and the intensity of fluctuations, transport is impossible in the absence of the additional force $\varphi(t)$. In the case of a constant force the result is self-evident because the heights of the potential barriers for the particle moving rightwards and leftwards are different. The case of an alternating force is more complicated. During one half-period the right potential barrier is lowered to $U_{0}-B x_{1}$, whereas the left one rises to $U_{0}+B x_{2}$. During the next half-period the right potential barrier rises to $U_{0}+B x_{1}$, whereas the left one is lowered to $U_{0}-B x_{2}$. Because the lowering of the potential barrier plays a dominant role, the particle moves, in average, in the direction of larger lowering of the potential barrier. 


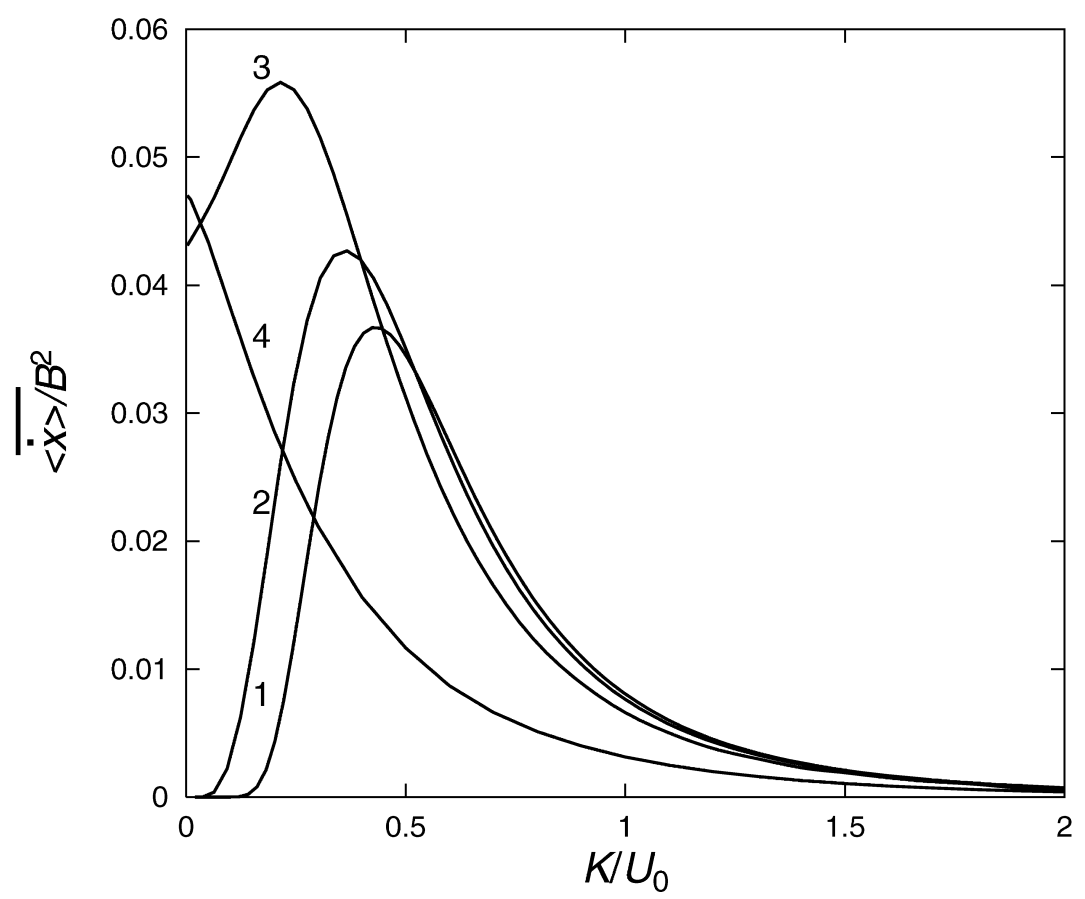

Fig. 5. Dependence of $\overline{\langle\dot{x}\rangle} / B^{2}$ on $K / U_{0}$ as described by Eqs. (3.7) and (3.13) for $a_{1}=1.25, a_{2}=5, x_{1}=0.8, x_{2}=0.2$, and: $B=0.1$ (curve 1$) ; B=1$ (curve 2 ); $B=2$ (curve 3 ); and $B=5$ (curve 4 ).

We note that a similar problem for $B_{0}=0$ was solved numerically in [81] by use of the so-called matrix continued fraction technique. It was shown that, for low frequencies, the numerical results coincide with quasistationary approximation (in [81] it is called the adiabatic approximation). But for high frequencies the results obtained were radically different in character; for example, a reversal of the probability flux over certain ranges of $K / U_{0}$ and $B$ was detected.

\subsubsection{The case of random modulation of the potential barrier height}

For simplicity assume that in Eq. (3.1) the regular force $\varphi(t)$ is absent. In this case a stationary solution of the Fokker-Planck equation (3.4) satisfying the continuity condition for $x=0$ is

$$
w(x)= \begin{cases}-\frac{G}{a_{1}}\left(1-\exp \left(-\frac{2 a_{1} x}{K^{(1)}}\right)\right)+C \exp \left(-\frac{2 a_{1} x}{K^{(1)}}\right) & \text { for } 0<x<x_{1}, \\ \frac{G}{a_{2}}\left(1-\exp \left(\frac{2 a_{2} x}{K^{(2)}}\right)\right)+C \exp \left(\frac{2 a_{2} x}{K^{(2)}}\right) & \text { for }-x_{2}<x<0,\end{cases}
$$

where

$$
K^{(1,2)}=K+K_{1} a_{1,2}^{2} .
$$


From the periodicity condition for the function $w(x)$ we find the relation between $G$ and $C$ :

$$
\begin{gathered}
G\left[a_{1}\left(1-\exp \left(-\frac{2 U_{0}}{K^{(2)}}\right)\right)+a_{2}\left(1-\exp \left(-\frac{2 U_{0}}{K^{(1)}}\right)\right)\right] \\
\quad=C a_{1} a_{2}\left[\exp \left(-\frac{2 U_{0}}{K^{(1)}}\right)-\exp \left(-\frac{2 U_{0}}{K^{(2)}}\right)\right] .
\end{gathered}
$$

Taking account of (3.30) and (3.31), and from the normalization condition, we find $G$ :

$$
\begin{aligned}
G= & \frac{2 a_{1}^{2} a_{2}^{2}}{a_{1}+a_{2}}\left[\exp \left(-\frac{2 U_{0}}{K^{(1)}}\right)-\exp \left(-\frac{2 U_{0}}{K^{(2)}}\right)\right]\left\{\left(a_{1}+a_{2}\right)\left(K+K_{1} a_{1} a_{2}\right)\left[1-\exp \left(-\frac{2 U_{0}}{K^{(1)}}\right)\right]\right. \\
& \left.\times\left[1-\exp \left(-\frac{2 U_{0}}{K^{(2)}}\right)\right]-2 U_{0}\left(a_{2}-a_{1}\right)\left[\exp \left(-\frac{2 U_{0}}{K^{(1)}}\right)-\exp \left(-\frac{2 U_{0}}{K^{(2)}}\right)\right]\right\}^{-1} \cdot
\end{aligned}
$$

It follows from (3.32) that $\langle\dot{x}\rangle=G L \neq 0$ only if $a_{1} \neq a_{2}$ and $K_{1} \neq 0$. The dependences of $\langle\dot{x}\rangle$ on $K_{1}$ for different values of $K / U_{0}$, and on $K / U_{0}$ for a number of fixed values of $K_{1}$, are shown in Fig. 6. It is interesting that the particle moves on average in the direction of the greater rate of the potential change. We draw attention to the fact that, for random modulation of the potential barrier, the directional diffusion of particles is possible even in the absence of thermal fluctuations $(K=0)$.

\subsubsection{The case of an additional random force of large correlation time}

Fluctuational transport of a Brownian particle in a viscous medium induced by thermal noise and a correlated random force that is a Markov process was studied in [79]. However, concrete results were obtained only for dichotomous and "kangaroo"-like processes. We suggest here a way of tackling this problem for the case where the correlated random force is the so-called Ornstein-Uhlenbeck process [88]. We can then write the following equations of motion:

$$
\begin{aligned}
& \dot{x}+f(x)=y+\xi(t), \\
& \dot{y}=-\gamma y+\xi_{1}(t),
\end{aligned}
$$

where $f(x)$ is determined by expression (3.2), and $\xi(t)$ and $\xi_{1}(t)$ are uncorrelated white noises with zero mean values and intensities equal to $K$ and $K_{1}$ respectively. The stationary probability density of the variable $y$ is independent of $x$ and can be easily calculated from the Fokker-Planck equation associated with Eq. (3.34). It is equal to

$$
p(y)=\sqrt{\frac{\gamma}{\pi K_{1}}} \exp \left(-\frac{\gamma y^{2}}{K_{1}}\right) .
$$

Let us calculate now the conditional probability density of the variable $x$ for a fixed value of $y$. In the quasistationary approximation, which is valid for sufficiently large correlation time of the process $y(t)$, this probability density $w(x \mid y)$ satisfies the following Fokker-Planck equation:

$$
G(y)=-(f(x)-y) w(x \mid y)-\frac{K}{2} \frac{\partial w(x \mid y)}{\partial x},
$$



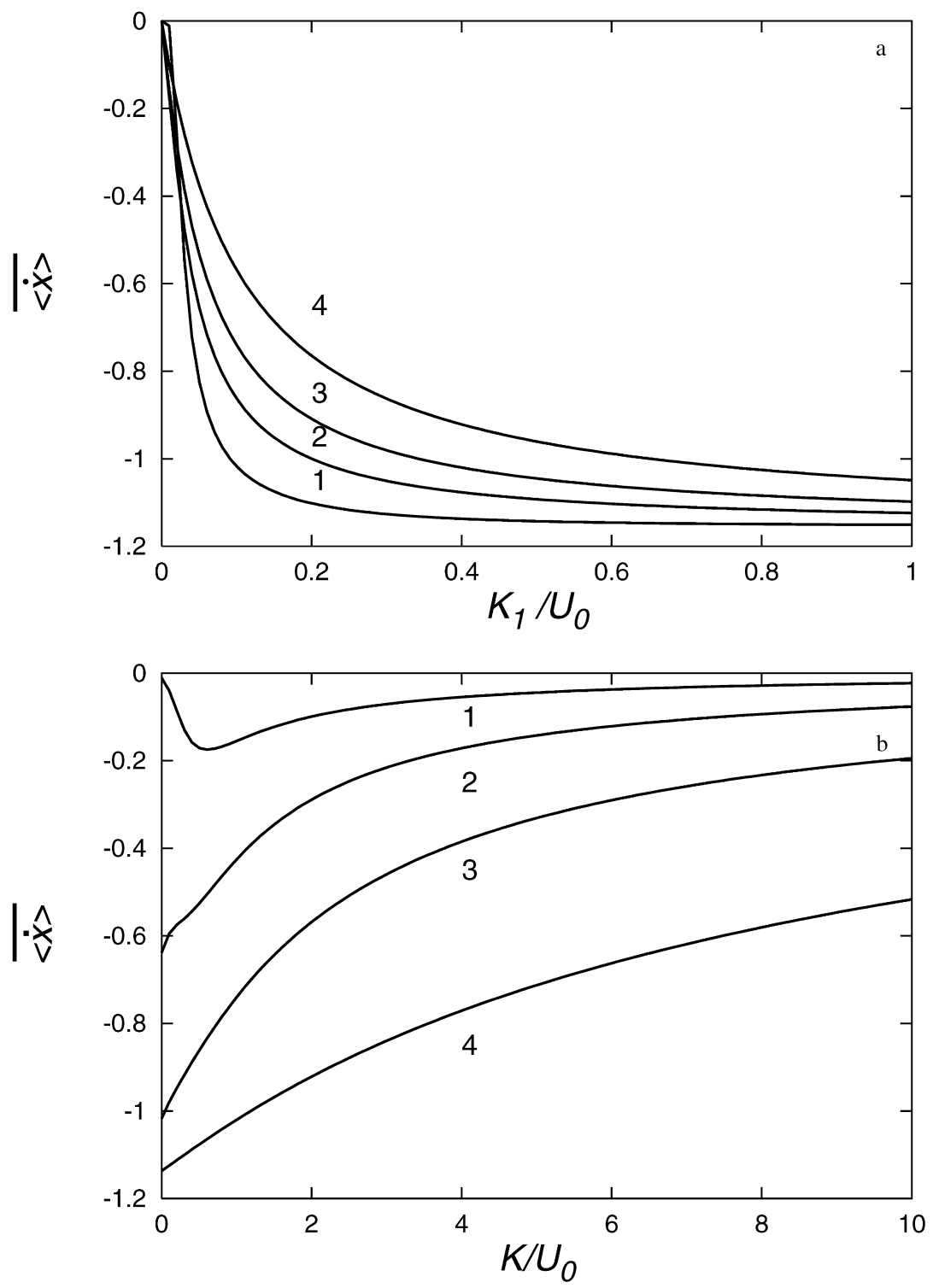

Fig. 6. Dependence of $\langle\dot{x}\rangle$ : (a) on $K_{1} / U_{0}$ for $K / U_{0}=0,0.5,1$, and 2 for curves $1-4$, respectively; and (b) on $K / U_{0}$ for $K_{1}=0.01,0.035,0.1$, and 0.4 for curves $1-4$, respectively. In both cases, $a_{1}=1.25$ and $a_{2}=5$.

where $G(y)$ is the probability flux for a fixed value of $y$. A solution of Eq. (3.36) is

$$
w(x \mid y)= \begin{cases}\frac{G(y)}{y-a_{1}}+\left(C(y)-\frac{G(y)}{y-a_{1}}\right) \exp \left(\frac{2\left(y-a_{1}\right)}{K} x\right) & \text { for } 0<x<x_{1}, \\ \frac{G(y)}{y+a_{2}}+\left(C(y)-\frac{G(y)}{y+a_{2}}\right) \exp \left(\frac{2\left(y+a_{2}\right)}{K} x\right) & \text { for }-x_{2}<x<0,\end{cases}
$$


where $C(y)$ is an arbitrary function of $y$. From the periodicity condition of the function $w(x \mid y)$ we find a relation between $G(y)$ and $C(y)$ :

$$
\begin{aligned}
C(y)= & \frac{G(y)}{q_{1} q_{2}}\left[q_{2} \exp \left(\frac{2 U_{0} y}{K a_{1}}\right)-q_{1} \exp \left(-\frac{2 U_{0} y}{K a_{2}}\right)-\left(a_{1}+a_{2}\right) \exp \left(\frac{2 U_{0}}{K}\right)\right] \\
& \times\left[\exp \left(\frac{2 U_{0} y}{K a_{1}}\right)-\exp \left(-\frac{2 U_{0} y}{K a_{2}}\right)\right]^{-1},
\end{aligned}
$$

where $q_{1,2}=y \mp a_{1,2}$. Taken together, (3.38) and the normalization condition yield the probability flux $G(y)$ :

$$
\begin{aligned}
G^{-1}(y)= & U_{0}\left(\frac{1}{a_{1} q_{1}}+\frac{1}{a_{2} q_{2}}\right)-\frac{K\left(a_{1}+a_{2}\right)^{2}}{2 q_{1}^{2} q_{2}^{2}} \exp \left(-\frac{2 U_{0}}{K}\right)\left[\exp \left(\frac{2 U_{0}}{K}\right)-\exp \left(-\frac{2 U_{0} y}{K a_{2}}\right)\right] \\
& \times\left[\exp \left(\frac{2 U_{0} y}{K a_{1}}\right)-\exp \left(\frac{2 U_{0}}{K}\right)\right]\left[\exp \left(\frac{2 U_{0} y}{K a_{1}}\right)-\exp \left(-\frac{2 U_{0} y}{K a_{2}}\right)\right]^{-1} .
\end{aligned}
$$

In analogy with the formula (3.24) we find that, for the quasistationary approximation considered,

$$
\langle\dot{x}\rangle=\langle G(y)\rangle L=\frac{a_{1}+a_{2}}{a_{1} a_{2}}\langle G(y)\rangle U_{0},
$$

The dependences of $\langle\dot{x}\rangle$ on $K_{1} / \gamma$ calculated numerically for $a_{1}=1.25, a_{2}=5$ and different values of $K / U_{0}$ are shown in Fig. 7(a). It is seen from this figure that for a fixed value of $K / U_{0}$ the value of $\langle\dot{x}\rangle$ first increases as $K_{1} / \gamma$ increases and then slowly decreases again, approaching zero as $K_{1} / \gamma \rightarrow \infty$. The peak of $\langle\dot{x}\rangle$ is located at greater values of $K_{1} / \gamma$, the greater is $K / U_{0}$. The dependencies of $\langle\dot{x}\rangle$ on $K / U_{0}$ for a fixed value of $K_{1} / \gamma$, shown in Fig. 7(b), are of a somewhat different form. They display maxima at a certain value of $K / U_{0} \neq 0$ only for $K_{1} / \gamma$ less than a critical value; whereas for greater $K_{1} / \gamma$ the dependences become monotonically decreasing.

\subsection{The effect of the potential shape}

It is interesting to find how the shape of the potential influences noise-induced transport. We illustrate this problem by the example of the case when a small additional harmonic force acts upon the particle. So, we consider the motion of a particle described by Eq. (3.1) for $\zeta \equiv 0, \varphi(t)=B \cos \omega t$, where $\omega$ is sufficiently small. In this case the probability density is

$$
w(x, t)=\left[C(\varphi(t))-\frac{2 G(\varphi(t))}{K} \int_{0}^{x} \exp \left(2\left(U\left(x^{\prime}\right)-\varphi(t) x^{\prime}\right) K\right) \mathrm{d} x^{\prime}\right] \exp \left(-\frac{2(U(x)-\varphi(t) x)}{K}\right),
$$



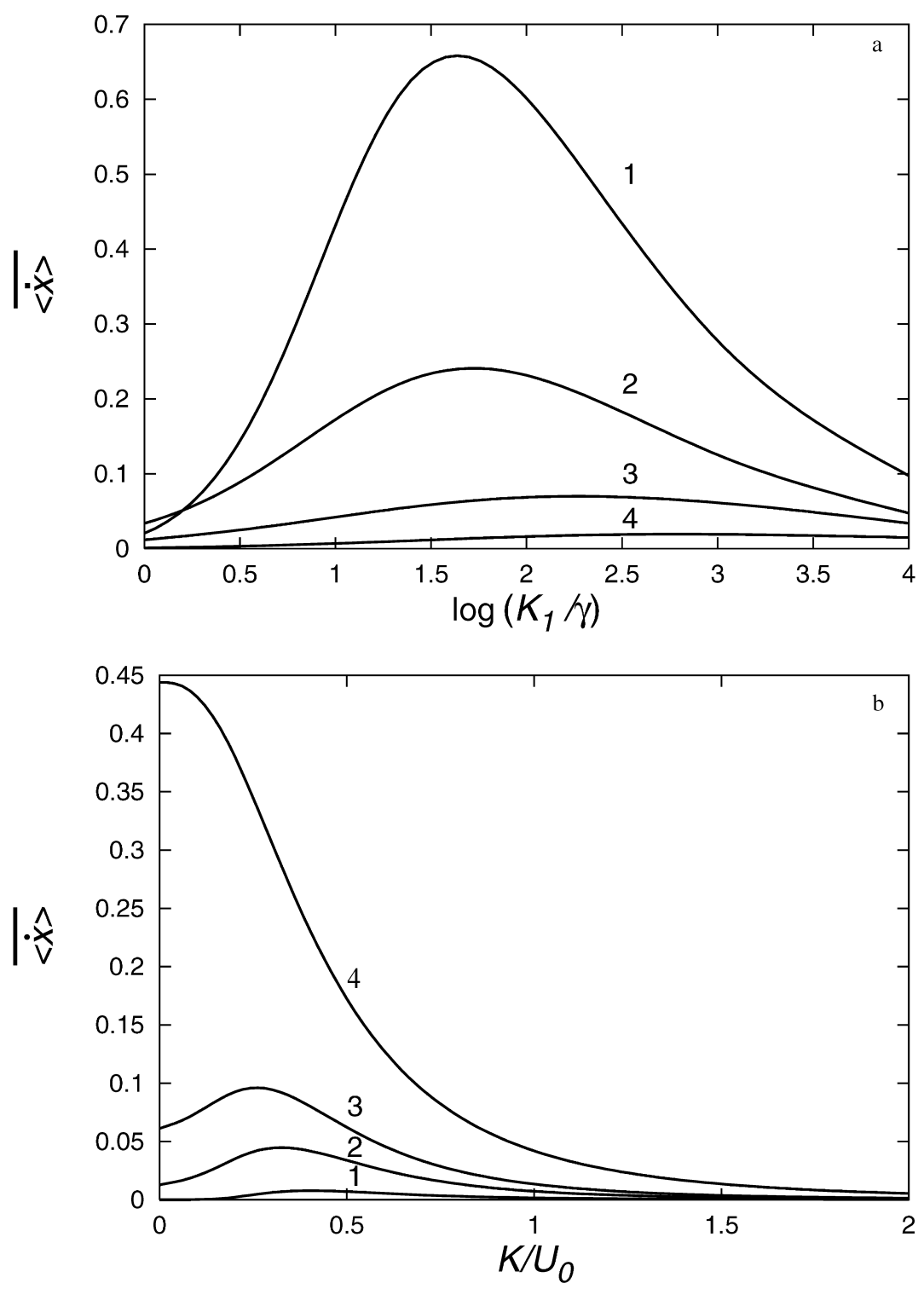

Fig. 7. Dependence of $\langle\dot{x}\rangle$ : (a) on $\log \left(K_{1} / \gamma\right)$ for $K / U_{0}=0.1,0.5,1$ and 2 for curves $1-4$, respectively; (b) on $K / U_{0}$ for $K_{1} / \gamma=0.2,1,2$, and 10 for curves $1-4$, respectively. In both cases, $U_{0}=1, L=1, a_{1}=1.25$ and $a_{2}=5$.

where $U(x)=\int_{0}^{x} f\left(x^{\prime}\right) \mathrm{d} x^{\prime}$ is the potential, and $C(\varphi(t))$ and $G(\varphi(t))$ are arbitrary functions of $t$. From the periodicity condition of the function $w(x, t)$ we find the relationship between $C(\varphi)$ and $G(\varphi)$ :

$$
C(\varphi)=\frac{2 G(\varphi) I_{1}(\varphi)}{K}\left[1-\exp \left(-\frac{2 L \varphi}{K}\right)\right]^{-1}
$$


where $L$ is the period of the functions $f(x)$ and $U(x)$,

$$
I_{1}(\varphi)=\int_{0}^{L} \exp \left(\frac{2(U(x)-\varphi x))}{K}\right) \mathrm{d} x .
$$

Taking account of (3.42) and from the normalization condition we determine $G(\varphi)$ :

$$
G(\varphi)=\frac{K}{2}\left\{I_{1}(\varphi) I_{2}(\varphi)\left[1-\exp \left(-\frac{2 L \varphi}{K}\right)\right]^{-1}-I_{3}(\varphi)\right\}^{-1},
$$

where

$$
\begin{aligned}
& I_{2}(\varphi)=\int_{0}^{L} \exp \left(-\frac{2(U(x)-\varphi x))}{K}\right) \mathrm{d} x, \\
& I_{3}(\varphi)=\int_{0}^{L} \int_{0}^{x} \exp \left(\frac{2\left(U\left(x^{\prime}\right)-U(x)-\varphi\left(x^{\prime}-x\right)\right)}{K}\right) \mathrm{d} x^{\prime} \mathrm{d} x .
\end{aligned}
$$

If $B$ satisfies to the condition

$$
L B \ll K,
$$

we find from (3.44)

$$
G(\varphi) \approx G_{01} \varphi+G_{02} \varphi^{2}, \quad I_{1}(\varphi) \approx I_{10}-I_{11} \varphi, \quad I_{2}(\varphi) \approx I_{20}+I_{21} \varphi, \quad I_{3}(\varphi) \approx I_{30},
$$

where

$$
\begin{aligned}
& G_{01}=\frac{L}{I_{10} I_{20}}, \quad G_{02}=G_{01}\left(\frac{I_{11}}{I_{10}}-\frac{I_{21}}{I_{20}}-\frac{L}{K}\left(1-\frac{2 I_{30}}{I_{10} I_{20}}\right)\right), \\
& I_{11}=\frac{2}{K} \int_{0}^{L} x \exp \left(\frac{2 U(x)}{K}\right) \mathrm{d} x, \quad I_{21}=\frac{2}{K} \int_{0}^{L} x \exp \left(-\frac{2 U(x)}{K}\right) \mathrm{d} x .
\end{aligned}
$$

By substituting (3.47) into (3.7) we obtain

$$
\overline{\langle\dot{x}\rangle} \approx \frac{L^{2} B^{2}}{2 I_{10} I_{20}}\left(\frac{I_{11}}{I_{10}}-\frac{I_{21}}{I_{20}}-\frac{L}{K}\left(1-\frac{2 I_{30}}{I_{10} I_{20}}\right)\right) .
$$

As the first example, we set the function $f(x)$ proportional to the first two terms of the Fourier series for $f(x)$ determined by (3.2), viz.

$$
\begin{aligned}
f(x)= & \sum_{n=1}^{2} \frac{1.1}{n \pi}\left(\left(a_{1}+a_{2}\right) \sin \frac{2 \pi n\left(x+x_{0}\right)}{L}-a_{1} \sin \frac{2 \pi n\left(x+x_{0}-x_{1}\right)}{L}\right. \\
& \left.-a_{2} \sin \frac{2 \pi n\left(x+x_{0}+x_{2}\right)}{L}\right),
\end{aligned}
$$

where $x_{0}$ is chosen so that $U(0)=0$. Plots of the functions $U(x)=\int_{0}^{x} f(x) \mathrm{d} x, f(x)$ and $f^{\prime}(x)$ for $x_{1}=0.8, x_{2}=0.2$ and $x_{0}=0.075$ are shown in Fig. 8(a). As the second example, we take the function $f(x)$ associated with a saw-tooth potential with smoothed edges. This function and the 

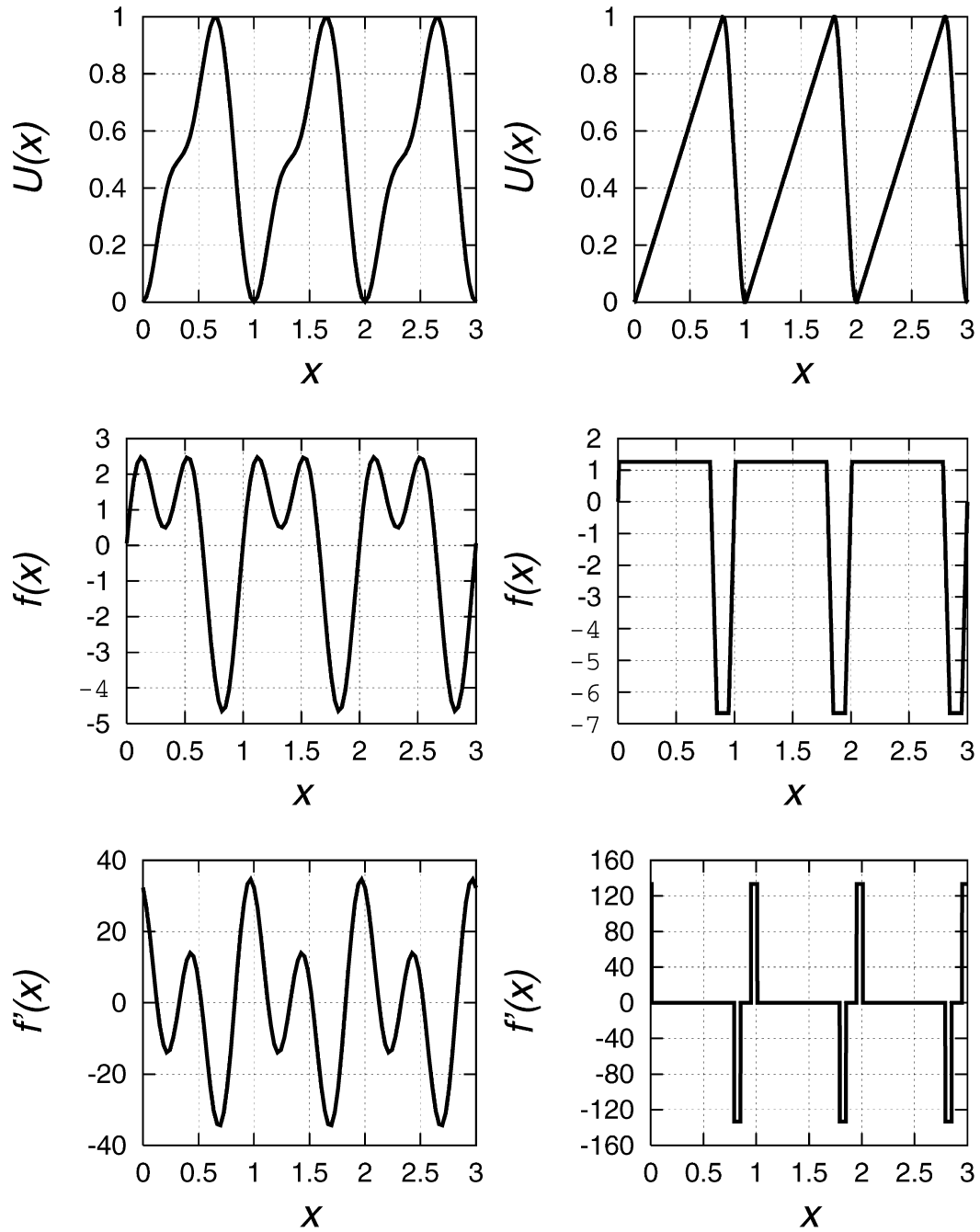

a

b

Fig. 8. Plots of the functions $U(x)=\int_{0}^{x} f(x) \mathrm{d} x, f(x)$ and $f^{\prime}(x)$ as determined by equations: (a) (3.50) for $a_{1}=1.25, a_{2}=5$, $x_{1}=0.8, x_{2}=0.2$, and $x_{0}=0.075$; (b) (3.51) and (3.52) for $x_{1}=0.8, x_{2}=0.2$, and $l_{2}=0.05\left(l_{1}=0.015, a_{1} \approx 1.27\right.$, $a_{2} \approx 6.67$, and $b \approx 133.33$ ).

corresponding potential $U(x)$ are described by the following expressions:

$$
f(x)=\left\{\begin{array}{cl}
b x & \text { for } 0 \leq x \leq l_{1}, \\
a_{1} & \text { for } l_{1} \leq x \leq x_{1}-l_{1}, \\
a_{1}-b\left(x-x_{1}+l_{1}\right) & \text { for } x_{1}-l_{1} \leq x \leq x_{1}+l_{2}, \\
-a_{2} & \text { for } x_{1}+l_{2} \leq x \leq L-l_{2}, \\
-a_{2}+b\left(x-L+l_{2}\right) & \text { for } L-l_{2} \leq x \leq L
\end{array}\right.
$$




$$
U(x)=\left\{\begin{array}{cl}
b x^{2} / 2 & \text { for } 0 \leq x \leq l_{1}, \\
a_{1}\left(x-\left(l_{1} / 2\right)\right) & \text { for } l_{1} \leq x \leq x_{1}-l_{1}, \\
1-b\left(x-x_{1}\right)^{2} / 2 & \text { for } x_{1}-l_{1} \leq x \leq x_{1}+l_{2} \\
1-a_{2}\left(x-x_{1}-\left(l_{2} / 2\right)\right) & \text { for } x_{1}+l_{2} \leq x \leq L-l_{2}, \\
b(x-L)^{2} / 2 & \text { for } L-l_{2} \leq x \leq L
\end{array}\right.
$$

where

$$
a_{1}=\frac{1}{x_{1}-l_{1}}, \quad a_{2}=\frac{1}{x_{2}-l_{2}}, \quad l_{1}=\frac{x_{1}}{2}-\sqrt{\frac{x_{1}^{2}}{4}-x_{2} l_{2}+l_{2}^{2}}, \quad b=\frac{a_{2}}{l_{2}},
$$

$x_{1}=0.8, x_{2}=0.2, L=1, l_{2}$ is a certain parameter that characterizes the extent to which the potential edges are smoothed. Plots of the functions $U(x), f(x)$ and $f^{\prime}(x)$ for $l_{2}=0.05$ $\left(l_{1} \approx 0.0095, a_{1} \approx 1.265, a_{2} \approx 6.67\right.$, and $\left.b \approx 133.333\right)$ are shown in Fig. 8(b).

For $f(x)$ described by the expressions (3.50) (for $a_{1}=1.25, a_{2}=5, x_{1}=0.8, x_{2}=0.2$ ) and (3.52) (for the same values of $x_{1}$ and $x_{2}$ and $l_{2}=0.05$ ) the dependences of $\overline{\langle\dot{x}\rangle} / B^{2}$ on $U_{0} / K$ are illustrated in Fig. 9 (the curves 2 and 3, respectively). For comparison, in the same figure the corresponding dependence for a nonsmoothed saw-tooth potential is shown too (the curve 1). We see that all of these dependencies coincide in a qualitative sense but diverge quantitatively.

\subsection{The effect of the particle mass}

We revert now to Eq. (3.3) assuming that $\mu$ is sufficiently small, viz.

$$
\mu \max f^{\prime}(x) \ll 1 \text {. }
$$

In this case we can obtain an approximate one-dimensional Fokker-Planck equation for the probability density of the variable $x$, much as this was done by Stratonovich [26]. The derivation of such an equation for the case that $\zeta(x, t) \equiv 0$ is given in Appendix A. For $\mu=0$ the equation found is the exact Fokker-Planck equation corresponding to the Langevin equation (3.1).

\subsubsection{The case of a small additional regular force}

Setting in Eq. (A.20) $\varepsilon^{2}=\mu$ and $F(x, t)=f(x)-\varphi(t)$, and retaining the terms up to the order 3 , inclusive, with respect to $\mu$, we obtain in the quasistationary approximation the following equation for $w(x, \varphi)$ :

$$
\begin{aligned}
G(\varphi)= & -\left\{1+\mu f^{\prime}(x)+\mu^{2}\left[\frac{3 K}{4} f^{\prime \prime \prime}(x)+2(f(x)-\varphi) f^{\prime \prime}(x)+2\left(f^{\prime}(x)\right)^{2}\right]\right. \\
& +\mu^{3}\left[\frac{29 K^{2}}{48} f^{\mathrm{V}}(x)+\frac{K}{8}\left(23(f(x)-\varphi) f^{\mathrm{IV}}(x)+64 f^{\prime}(x) f^{\prime \prime \prime}(x)+31\left(f^{\prime \prime}(x)\right)^{2}\right)\right. \\
& \left.\left.+\frac{7}{2}(f(x)-\varphi)^{2} f^{\prime \prime \prime}(x)+16(f(x)-\varphi) f^{\prime}(x) f^{\prime \prime}(x)+5\left(f^{\prime}(x)\right)^{3}\right]\right\} \\
& \times\left(\frac{K}{2} \frac{\partial w}{\partial x}+(f(x)-\varphi) w\right),
\end{aligned}
$$




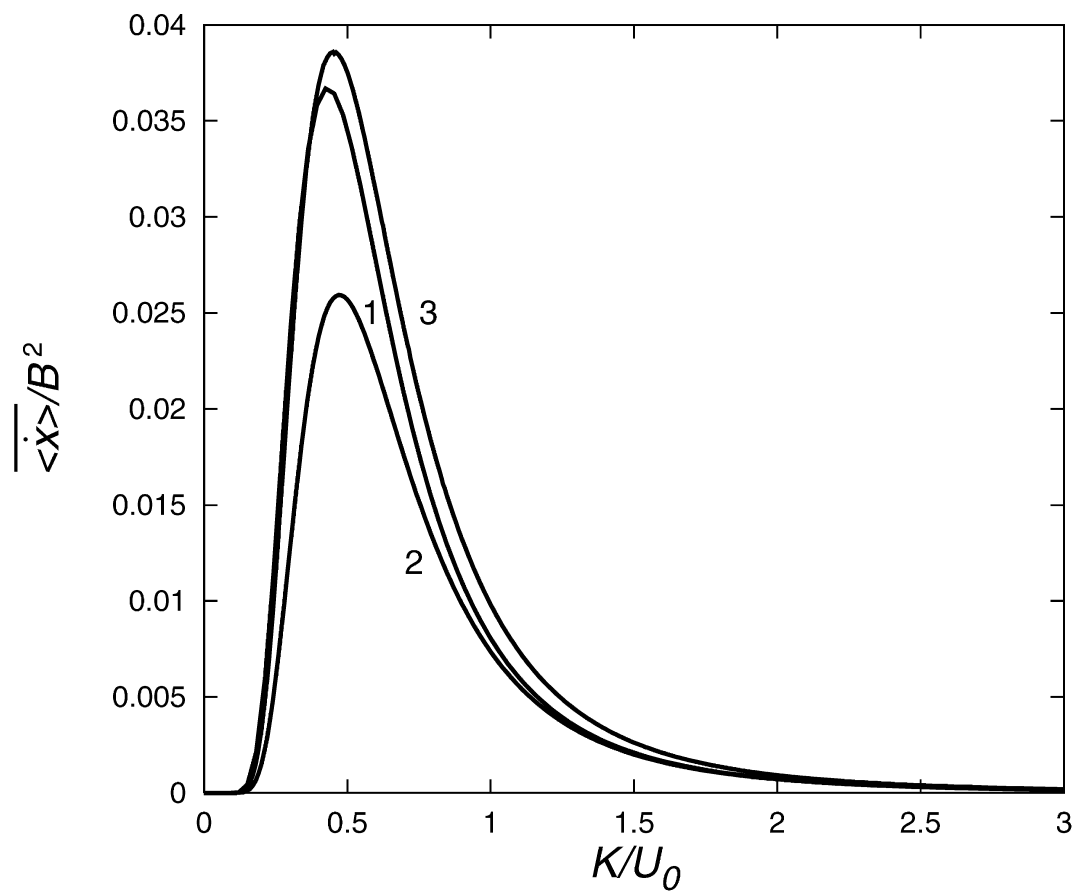

Fig. 9. Dependence of $\overline{\langle\dot{x}\rangle} / B^{2}$ on $U_{0} / K$ for $f(x)$ described by (3.2) (curve 1), (3.50) (curve 2), and (3.52) (curve 3) for the same values of the parameters as in Fig. 8.

where $G(\varphi)$ is the probability flux at a fixed value of $\varphi$. A solution of Eq. (3.54) is

$$
\begin{aligned}
w(\varphi)= & \exp \left(-\frac{2(U(x)-\varphi x)}{K}\right)\left\{C(\varphi)-\frac{2 G(\varphi)}{K} \int_{0}^{x}\left(\left(1-\mu f^{\prime}\left(x^{\prime}\right)-\mu^{2}\left[\frac{3 K}{4} f^{\prime \prime \prime}(x)\right.\right.\right.\right. \\
& \left.+2(f(x)-\varphi) f^{\prime \prime}(x)+\left(f^{\prime}(x)\right)^{2}\right]-\mu^{3}\left[\frac{29 K^{2}}{48} f^{\mathrm{v}}(x)+\frac{K}{8}\left(23(f(x)-\varphi) f^{\mathrm{IV}}(x)\right.\right. \\
& \left.+52 f^{\prime}(x) f^{\prime \prime \prime}(x)+31\left(f^{\prime \prime}(x)\right)^{2}\right)+\frac{7}{2}(f(x)-\varphi)^{2} f^{\prime \prime \prime}(x)+12(f(x)-\varphi) f^{\prime}(x) f^{\prime \prime}(x) \\
& \left.\left.\left.+2\left(f^{\prime}(x)\right)^{3}\right]\right) \exp \left(\frac{2\left(U\left(x^{\prime}\right)-\varphi x^{\prime}\right)}{K}\right) \mathrm{d} x^{\prime}\right\} .
\end{aligned}
$$

From the periodicity condition of the function $w(x, \varphi)$ we find the relationship between $C(\varphi)$ and $G(\varphi)$ :

$$
\begin{aligned}
C(\varphi)= & \frac{2 G(\varphi)}{K}\left(I_{1}(\varphi)-\mu I_{4}(\varphi)-\mu^{2}\left(I_{6}(\varphi)-I_{7}(\varphi) \varphi\right)\right. \\
& \left.-\mu^{3}\left(I_{12}(\varphi)-I_{13}(\varphi) \varphi+I_{14}(\varphi) \varphi^{2}\right)\right)\left[1-\exp \left(-\frac{2 L \varphi}{K}\right)\right]^{-1},
\end{aligned}
$$


where $I_{1}(\varphi)$ is determined by (3.43),

$$
\begin{aligned}
I_{4}(\varphi)= & \int_{0}^{L} f^{\prime}(x) \exp \left(\frac{2(U(x)-\varphi x)}{K}\right) \mathrm{d} x, \quad I_{7}(\varphi)=2 \int_{0}^{L} f^{\prime \prime}(x) \exp \left(\frac{2(U(x)-\varphi x)}{K}\right) \mathrm{d} x, \\
I_{6}(\varphi)= & \int_{0}^{L}\left(\frac{3 K}{4} f^{\prime \prime \prime}(x)+2 f(x) f^{\prime \prime}(x)+\left(f^{\prime}(x)\right)^{2}\right) \exp \left(\frac{2(U(x)-\varphi x)}{K}\right) \mathrm{d} x, \\
I_{12}(\varphi)= & \int_{0}^{L}\left[\frac{29 K^{2}}{48} f^{\mathrm{V}}(x)+\frac{K}{8}\left(23 f(x) f^{\mathrm{IV}}(x)+52 f^{\prime}(x) f^{\prime \prime \prime}(x)+31\left(f^{\prime \prime}(x)\right)^{2}\right)\right. \\
& \left.+\frac{7}{2} f^{2}(x) f^{\prime \prime \prime}(x)+12 f(x) f^{\prime}(x) f^{\prime \prime}(x)+2\left(f^{\prime}(x)\right)^{3}\right] \exp \left(\frac{2(U(x)-\varphi x)}{K}\right) \mathrm{d} x, \\
I_{13}(\varphi)= & \int_{0}^{L}\left(\frac{23 K}{8} f^{\mathrm{IV}}(x)+7 f(x) f^{\prime \prime \prime}(x)+12 f^{\prime}(x) f^{\prime \prime}(x)\right) \exp \left(\frac{2(U(x)-\varphi x)}{K}\right) \mathrm{d} x, \\
I_{14}(\varphi)= & \frac{7}{2} \int_{0}^{L} f^{\prime \prime \prime}(x) \exp \left(\frac{2(U(x)-\varphi x)}{K}\right) \mathrm{d} x .
\end{aligned}
$$

Taking account of (3.56), and from the normalization condition, we find $G(\varphi)$. It can be written as

$$
G(\varphi)=G_{0}(\varphi)\left(1+\mu M_{1}(\varphi)+\mu^{2} M_{2}(\varphi)+\mu^{3} M_{3}(\varphi)\right),
$$

where $G_{0}(\varphi)$ is determined by the formula (3.44), and

$$
\begin{aligned}
& M_{1}(\varphi)=\frac{I_{2}(\varphi) I_{4}(\varphi)-I_{5}(\varphi)(1-\exp (-2 L \varphi / K))}{I_{1}(\varphi) I_{2}(\varphi)-I_{3}(\varphi)(1-\exp (-2 L \varphi / K))}, \\
& M_{2}(\varphi)=M_{1}^{2}(\varphi)+\frac{I_{2}(\varphi)\left(I_{6}(\varphi)-I_{7}(\varphi) \varphi\right)-\left(I_{8}(\varphi)-I_{9}(\varphi) \varphi\right)(1-\exp (-2 L \varphi / K))}{I_{1}(\varphi) I_{2}(\varphi)-I_{3}(\varphi)(1-\exp (-2 L \varphi / K))} \\
& M_{3}(\varphi) \\
& =2 M_{1}(\varphi) M_{2}(\varphi)-M_{1}^{3}(\varphi) \\
& \quad \times \frac{I_{2}(\varphi)\left(I_{12}(\varphi)-I_{13}(\varphi) \varphi+I_{14}(\varphi) \varphi^{2}\right)-\left(I_{15}(\varphi)-I_{16}(\varphi) \varphi+I_{17}(\varphi) \varphi^{2}\right)(1-\exp (-2 L \varphi / K))}{I_{1}(\varphi) I_{2}(\varphi)-I_{3}(\varphi)(1-\exp (-2 L \varphi / K))} .
\end{aligned}
$$

$I_{2}(\varphi), I_{3}(\varphi)$ are determined by (3.45),

$$
\begin{aligned}
I_{5}(\varphi)= & \int_{0}^{L} \int_{0}^{x} f^{\prime}\left(x^{\prime}\right) \exp \left(\frac{2\left(U\left(x^{\prime}\right)-U(x)-\varphi(t)\left(x^{\prime}-x\right)\right)}{K}\right) \mathrm{d} x^{\prime} \mathrm{d} x, \\
I_{8}(\varphi)= & \int_{0}^{L} \int_{0}^{x}\left(\frac{3 K}{4} f^{\prime \prime \prime}\left(x^{\prime}\right)+2 f\left(x^{\prime}\right) f^{\prime \prime}\left(x^{\prime}\right)+\left(f^{\prime}\left(x^{\prime}\right)\right)^{2}\right) \\
& \times \exp \left(\frac{2\left(U\left(x^{\prime}\right)-U(x)-\varphi\left(x^{\prime}-x\right)\right)}{K}\right) \mathrm{d} x^{\prime} \mathrm{d} x, \\
I_{9}(\varphi)= & 2 \int_{0}^{L} \int_{0}^{x} f^{\prime \prime}\left(x^{\prime}\right) \exp \left(\frac{2\left(U\left(x^{\prime}\right)-U(x)-\varphi\left(x^{\prime}-x\right)\right)}{K}\right) \mathrm{d} x^{\prime} \mathrm{d} x,
\end{aligned}
$$




$$
\begin{aligned}
I_{15}(\varphi)= & \int_{0}^{L} \int_{0}^{x}\left[\frac{29 K^{2}}{48} f^{\mathrm{V}}\left(x^{\prime}\right)+\frac{K}{8}\left(23 f\left(x^{\prime}\right) f^{\mathrm{IV}}\left(x^{\prime}\right)+52 f^{\prime}\left(x^{\prime}\right) f^{\prime \prime \prime}\left(x^{\prime}\right)+31\left(f^{\prime \prime}\left(x^{\prime}\right)\right)^{2}\right)\right. \\
& \left.+\frac{7}{2} f^{2}\left(x^{\prime}\right) f^{\prime \prime \prime}\left(x^{\prime}\right)+12 f\left(x^{\prime}\right) f^{\prime}\left(x^{\prime}\right) f^{\prime \prime}\left(x^{\prime}\right)+2\left(f^{\prime}\left(x^{\prime}\right)\right)^{3}\right] \\
& \times \exp \left(\frac{2\left(U\left(x^{\prime}\right)-U(x)-\varphi\left(x^{\prime}-x\right)\right)}{K}\right) \mathrm{d} x^{\prime} \mathrm{d} x, \\
I_{16}(\varphi)= & \int_{0}^{L} \int_{0}^{x}\left(\frac{23 K}{8} f^{\mathrm{IV}}\left(x^{\prime}\right)+7 f\left(x^{\prime}\right) f^{\prime \prime \prime}\left(x^{\prime}\right)+12 f^{\prime}\left(x^{\prime}\right) f^{\prime \prime}\left(x^{\prime}\right)\right) \\
& \times \exp \left(\frac{2\left(U\left(x^{\prime}\right)-U(x)-\varphi\left(x^{\prime}-x\right)\right)}{K}\right) \mathrm{d} x^{\prime} \mathrm{d} x, \\
I_{17}(\varphi)= & \int_{0}^{L} \int_{0}^{x} \frac{7}{2} f^{\prime \prime \prime}\left(x^{\prime}\right) \exp \left(\frac{2\left(U\left(x^{\prime}\right)-U(x)-\varphi\left(x^{\prime}-x\right)\right)}{K}\right) \mathrm{d} x^{\prime} \mathrm{d} x .
\end{aligned}
$$

If $\varphi$ is sufficiently small then $G_{0}(\varphi)=G_{01} \varphi+G_{02} \varphi^{2}$, where $G_{01}$ and $G_{02}$ are determined by (3.48), and

$$
\begin{aligned}
& M_{i}(\varphi)=M_{i 0}+M_{i 1} \varphi \quad(i=1,2,3), \\
& M_{10}=\frac{I_{40}}{I_{10}}, \quad M_{11}=-M_{10}\left[\frac{I_{41}}{I_{40}}-\frac{I_{11}}{I_{10}}+\frac{2 L}{K I_{20}}\left(\frac{I_{50}}{I_{40}}-\frac{I_{30}}{I_{10}}\right)\right], \\
& M_{20}=M_{10}^{2}+\frac{I_{60}}{I_{10}}, \quad M_{21}=2 M_{10} M_{11}-\frac{I_{60}}{I_{10}}\left[\frac{I_{70}}{I_{60}}+\frac{I_{61}}{I_{60}}-\frac{I_{11}}{I_{10}}+\frac{2 L}{K I_{20}}\left(\frac{I_{80}}{I_{60}}-\frac{I_{30}}{I_{10}}\right)\right] \text {, } \\
& M_{30}=\frac{I_{40}^{3}}{I_{10}^{3}}+\frac{2 I_{40} I_{60}}{I_{10}^{2}}+\frac{I_{120}}{I_{10}} \\
& M_{31}=2\left(M_{10} M_{21}+\frac{I_{60}}{I_{10}} M_{11}\right)-M_{10}^{2} M_{11} \\
& -\frac{I_{120}}{I_{10}}\left[\frac{I_{121}}{I_{120}}+\frac{I_{130}}{I_{120}}-\frac{I_{11}}{I_{10}}+\frac{2 L}{K I_{20}}\left(\frac{I_{150}}{I_{120}}-\frac{I_{30}}{I_{10}}\right)\right] \text {, } \\
& I_{40}=\left.I_{4}\right|_{\varphi=0}, \quad I_{50}=\left.I_{5}\right|_{\varphi=0}, \quad I_{41}=\frac{2}{K} \int_{0}^{L} x f^{\prime}(x) \exp \left(\frac{2 U(x)}{K}\right) \mathrm{d} x, \\
& I_{60}=\left.I_{6}\right|_{\varphi=0}, \quad I_{70}=\left.I_{7}\right|_{\varphi=0}, \quad I_{80}=\left.I_{8}\right|_{\varphi=0}, \\
& I_{61}=\frac{2}{K} \int_{0}^{L} x\left(\frac{3 K}{4} f^{\prime \prime \prime}(x)+2 f(x) f^{\prime \prime}(x)+\left(f^{\prime}(x)\right)^{2}\right) \exp \left(\frac{2 U(x)}{K}\right) \mathrm{d} x, \\
& I_{120}=\left.I_{12}\right|_{\varphi=0}, \quad I_{130}=\left.I_{13}\right|_{\varphi=0}, \quad I_{150}=\left.I_{15}\right|_{\varphi=0}, \\
& I_{121}=\frac{2}{K} \int_{0}^{L} x\left[\frac{29 K^{2}}{48} f^{\mathrm{v}}(x)+\frac{K}{8}\left(23 f(x) f^{\mathrm{IV}}(x)+52 f^{\prime}(x) f^{\prime \prime \prime}(x)+31\left(f^{\prime \prime}(x)\right)^{2}\right)\right. \\
& \left.+\frac{7}{2} f^{2}(x) f^{\prime \prime \prime}(x)+12 f(x) f^{\prime}(x) f^{\prime \prime}(x)+2\left(f^{\prime}(x)\right)^{3}\right] \exp \left(\frac{2 U(x)}{K}\right) \mathrm{d} x .
\end{aligned}
$$


If $\varphi(t)=B \cos \omega t+B_{0}$ then

$$
\begin{aligned}
\overline{\langle\dot{x}\rangle} \approx & \frac{B^{2} L}{2}\left[G_{02}+\mu\left(G_{01} M_{11}+G_{02} M_{10}\right)+\mu^{2}\left(G_{01} M_{21}+G_{02} M_{20}\right)\right. \\
& \left.+\mu^{3}\left(G_{01} M_{31}+G_{02} M_{30}\right)\right]+B_{0} L G_{01}\left(1+\mu M_{10}+\mu^{2} M_{20}+\mu^{3} M_{30}\right) .
\end{aligned}
$$

For $f(x)$ described by the formula (3.52), with $x_{1}=0.8, x_{2}=0.2$ and $l_{2}=0.05$, the dependencies of $\overline{\langle\dot{x}\rangle} / B^{2}$ on $K / U_{0}$ for different values of $\mu$ are shown in Fig. 10 . We see that the third approximation gives the flux reversal both at moderately large values of $K / U_{0}\left(K / U_{0}>3\right)$ and at moderately small values of $K / U_{0}\left(K / U_{0}<1\right)$. In the second approximation only one flux reversal retains, for $K / U_{0}>3$. It follows from here that for the verification of the results obtained a consideration of higher approximations is necessary.

It is evident that the dependence of the mean velocity of a particle on its mass can be used for the separation of particles of different masses. Examples of the dependencies of $\overline{\langle\dot{x}\rangle} / B^{2}$ on $\mu$ for different values of $K / U_{0}$ calculated in the first, second and third approximations are illustrated in Fig. 11. We see that the difference between the results is small only for $\mu<0.002$ but for such values of $\mu$ the flux reversal is possible only for large values of $K / U_{0}$.

We note that the results reported in this section coincide qualitatively with the corresponding results of [86].

\section{Noise-induced phase transitions in nonlinear oscillators}

In recent years the problem of nonequilibrium noise-induced phase transitions has attracted considerable attention from many scientists. These transitions are characterized by a qualitative change of the state of a system as the intensity of noise acting upon it increases. This change can manifest itself in the appearance of new extrema in the probability distribution for the system variables or disappearance of old ones [2,89], in either stabilization or destabilization of system equilibrium states [1,9], in the occurrence of so-called mean field [4-7], in the excitation of oscillations $[1,8,9,11,10]$, in the appearance of supernarrow spectral peaks close to the transition [90,91], and other marked changes in behaviour.

\subsection{Noise-induced multistability and multimodality}

Horsthemke and Lefever discuss [2] many examples where additional peaks appear in the probability density under influence of multiplicative noise. This phenomenon is treated as a nonequilibrium noise-induced phase transition (NIPT). In so doing it is correctly reasoned that the appearance of a peak in the probability density signals the appearance of a new stable steady state, i.e., the occurrence of multistability or multimodality. NIPTs of this type were first demonstrated convincingly in the genetic model equation [3] and subsequently in a cubic bistable system [92].

We consider here one example of noise-induced multistability: the pendulum with a randomly vibrated axis of suspension, for which the unstable upper equilibrium position can be transformed by noise to a stable one. It is well known that, where the suspension is vibrated harmonically, the upper equilibrium position can become stable if the frequency of the vibration is sufficiently high 

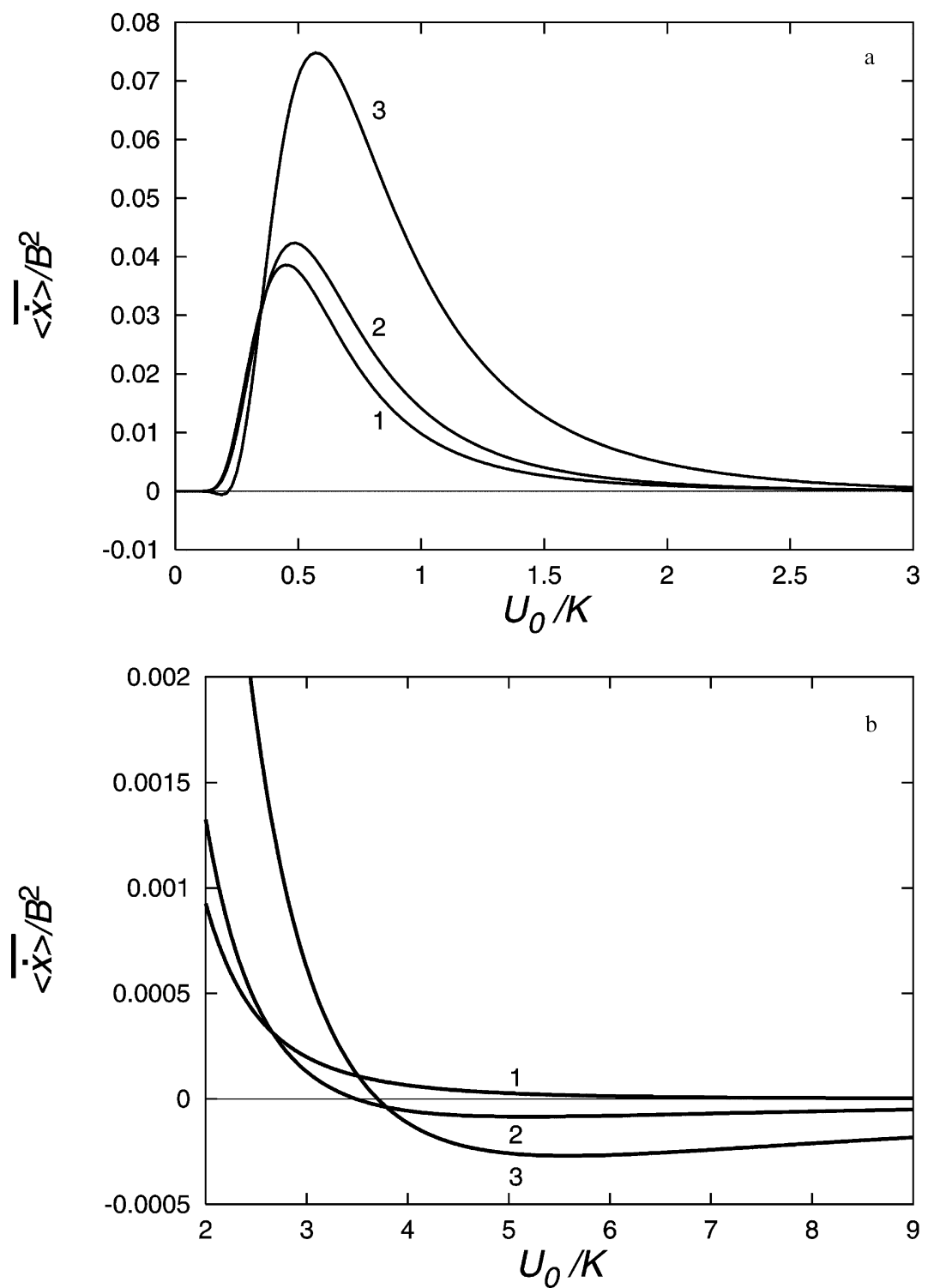

Fig. 10. The dependence of $\overline{\langle\dot{x}\rangle} / B^{2}$ on $K / U_{0}$ for $f(x)$ described by formula (3.51) for $\mu=0,0.005$ and 0.01 for curves $1-3$, respectively: (a) in the range $0 \leq K / U_{0} \leq 3$; and (b) in the range $2 \leq K / U_{0} \leq 9$.

(see, for example, [49,87,1]). This phenomenon was observed experimentally by Kapitsa [93,94]. More recently, it was shown [8,9] that a similar phenomenon can be also occur in the case of random, but sufficiently high-frequency, vibration of the suspension axis.

The equation describing the oscillations of a pendulum with randomly vibrated suspension axis and additive noise can be written as

$$
\ddot{\varphi}+2 \beta \dot{\varphi}+\left(1+\omega^{2} \zeta(t)\right) \sin \varphi=\xi(t),
$$



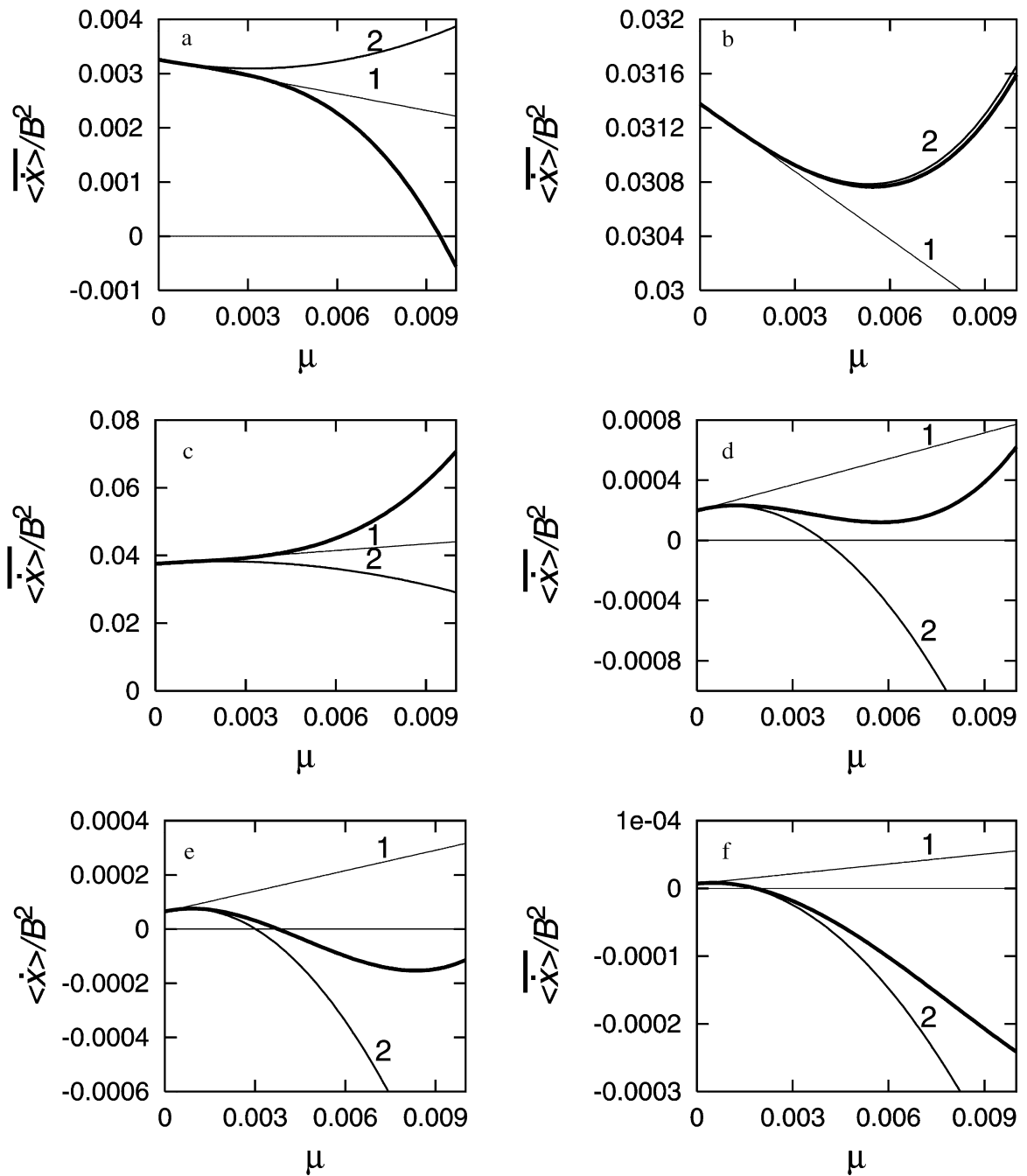

Fig. 11. The dependencies of $\overline{\langle\dot{x}\rangle} / B^{2}$ on $\mu$ for (a) $K / U_{0}=0.2$, (b) $K / U_{0}=0.35$, (c) $K / U_{0}=0.5$, (d) $K / U_{0}=3$, (e) $K / U_{0}=4$ and (f) $K / U_{0}=7$. The results obtained in the first and second approximations with respect to $\mu$ are labelled 1 and 2 , respectively.

where $\omega^{2} \zeta(t)$ is the acceleration of the pendulum suspension axis in terms of the acceleration of gravity, $\xi(t)$ is sufficiently wide-band random process with a negligibly small spectral density at the frequency $\omega$. Let $\zeta(t)$ be coloured narrow-band noise described by the equation

$$
\ddot{\zeta}+2 \alpha \dot{\zeta}+\omega^{2} \zeta=\chi(t),
$$

where $\chi(t)$ is white noise of intensity $\kappa_{1}, 1 \ll \alpha \ll \omega$. In this case the correlation function of the process $\zeta(t)$ is

$$
\langle\zeta(t) \zeta(t+\tau)\rangle \approx \sigma^{2} \mathrm{e}^{-\alpha \tau} \cos \omega \tau,
$$


where $\sigma^{2}=\kappa_{1} / 2 \omega^{2} \alpha$ is the variance of $\zeta(t)$. First we put $\xi(t) \equiv 0$ and show directly that the pendulum upper equilibrium state becomes stable if $\sigma^{2}$ is sufficiently large. If the power spectrum of the random process $\zeta(t)$ does not contain components in zones of parametric resonance, fluctuations of the variable $\varphi$ caused by the random vibration of the suspension axis are small. Putting $\varphi=\langle\varphi\rangle+\delta \varphi$, where $\delta \varphi \ll\langle\varphi\rangle$, we obtain from (4.1)

$$
\begin{aligned}
& \langle\ddot{\varphi}\rangle+2 \beta\langle\dot{\varphi}\rangle+\sin \langle\varphi\rangle+\omega^{2} \cos \langle\varphi\rangle\langle\zeta(t) \delta \varphi\rangle=0, \\
& \delta \ddot{\varphi}+2 \beta \delta \dot{\varphi}+\cos \langle\varphi\rangle \delta \varphi+\omega^{2} \zeta(t) \sin \langle\varphi\rangle=0 .
\end{aligned}
$$

A steady-state solution of Eqs. (4.4) and (4.5), having the form

$$
\langle\varphi\rangle=\pi, \quad \delta \varphi=0,
$$

corresponds to the upper equilibrium position of the pendulum, in whose stability we are interested. To investigate this stability, we can linearize Eqs. (4.4) and (4.5) with respect to small deviations from the solution (4.6) $\psi=\langle\varphi\rangle-\pi$ and $\delta \varphi$. The linearized equations are

$$
\begin{aligned}
& \ddot{\psi}+2 \beta \dot{\psi}-\psi-\omega^{2}\langle\zeta(t) \delta \varphi\rangle=0, \\
& \delta \ddot{\varphi}+2 \beta \delta \dot{\varphi}-\delta \varphi-\omega^{2} \zeta(t) \psi=0 .
\end{aligned}
$$

A steady-state solution of Eq. (4.8) is

$$
\delta \varphi(t)=\frac{\omega^{2}}{2 \sqrt{1+\beta^{2}}} \int_{-\infty}^{t}\left[\exp \left(p_{1}\left(t-t^{\prime}\right)\right)-\exp \left(p_{2}\left(t-t^{\prime}\right)\right)\right] \zeta\left(t^{\prime}\right) \psi\left(t^{\prime}\right) \mathrm{d} t^{\prime},
$$

where $p_{1,2}=-\beta \pm \sqrt{1+\beta^{2}}$ are the roots of the characteristic equation $p^{2}+2 \beta p-1=0$. From here we find

$$
\langle\zeta(t) \delta \varphi\rangle=\frac{\omega^{2}}{2 \sqrt{1+\beta^{2}}} \int_{-\infty}^{t}\left[\exp \left(p_{1}\left(t-t^{\prime}\right)\right)-\exp \left(p_{2}\left(t-t^{\prime}\right)\right)\right]\left\langle\zeta(t) \zeta\left(t^{\prime}\right)\right\rangle \psi\left(t^{\prime}\right) \mathrm{d} t^{\prime} .
$$

Putting in this expression $t^{\prime}-t=\tau$ and taking into account that the value $\psi$ does not vary significantly during the correlation time of the random process $\zeta(t)$, we rewrite (4.10) in the following form:

$$
\langle\zeta(t) \delta \varphi\rangle=-\frac{\omega^{2}}{2 \sqrt{1+\beta^{2}}} \psi(t) \int_{0}^{\infty}\left(\mathrm{e}^{-p_{1} \tau}-\mathrm{e}^{-p_{2} \tau}\right)\langle\zeta(t) \zeta(t+\tau)\rangle \mathrm{d} \tau .
$$

Substituting (4.3) into this expression and calculating the integral we obtain

$$
\langle\zeta(t) \delta \varphi\rangle=-\frac{\sigma^{2} \omega_{2}\left(\omega^{2}-\left(p_{1}+\alpha\right)\left(p_{2}+\alpha\right)\right)}{\left(\omega^{2}+\left(p_{1}+\alpha\right)^{2}\right)\left(\omega^{2}+\left(p_{2}+\alpha\right)^{2}\right)} \psi(t) .
$$

Because $\omega \gg 1, \beta, \alpha$, we have

$$
\langle\zeta(t) \delta \varphi\rangle \approx-\sigma^{2} \psi(t) .
$$

Substituting (4.13) into (4.7) we obtain for $\psi(t)$ the following approximate equation:

$$
\ddot{\psi}+2 \beta \dot{\psi}+\Omega_{0}^{2} \psi=0,
$$


where $\Omega_{0}=\sqrt{\omega^{2} \sigma^{2}-1}$ is the natural frequency of small oscillations of the pendulum about the upper equilibrium position. It follows from (4.14) that the mean deviation of the pendulum from its upper equilibrium position will decay, i.e., the equilibrium position will be stable, if the frequency $\Omega_{0}$ is real. This condition is valid if

$$
\omega^{2} \sigma^{2} \geq 1 \text {. }
$$

Let us show further that at condition (4.15) an additional maximum and minimum appear in the probability distribution. It follows from Eq. (4.1) that there is no stationary probability distribution for the original variables $\varphi$ and $\dot{\varphi}$. Therefore we use the "slow" variable $\phi=\langle\varphi\rangle$ and the "fast" variable $\delta \varphi$, which are described by Eq. (4.5), and include the additive noise $\xi(t)$. Taking into account the fact that $\zeta(t)$ is a narrow-band random process, which can be represented as

$$
\zeta(t)=\zeta_{1}(t) \cos \omega t+\zeta_{2}(t) \sin \omega t,
$$

where $\zeta_{1}(t)$ and $\zeta_{2}(t)$ are "slow" variables, we can put

$$
\delta \varphi=A(t) \cos \omega t+B(t) \sin \omega t,
$$

where $A(t)$ and $B(t)$ are "slow" variables. Substituting $\varphi(t)=\phi(t)+\delta \varphi(t)$, in view of (4.17), into Eq. (4.1), taking into account (4.16), and equating the slowly varying component and the coefficients of $\cos \omega t$ and $\sin \omega t$, we obtain the following equations for $\phi, A$ and $B$ :

$$
\begin{aligned}
& \ddot{\phi}+2 \beta \dot{\phi}+\sin \phi+\frac{\omega^{2} \cos \phi}{2}\left\langle A \zeta_{1}+B \zeta_{2}\right\rangle=\xi(t), \\
& \left(\omega^{2}-\cos \phi\right) A-2 \beta \omega B=\omega^{2} \zeta_{1} \sin \phi, \quad 2 \beta \omega A+\left(\omega^{2}-\cos \phi\right) B=\omega^{2} \zeta_{2} \sin \phi .
\end{aligned}
$$

Because $\omega \gg 1$, $\beta$, we find from Eqs. (4.19)

$$
A \approx \zeta_{1} \sin \phi, \quad B \approx \zeta_{2} \sin \phi .
$$

Substituting further (4.20) into Eq. (4.18) we obtain for $\phi(t)$ the following equation:

$$
\ddot{\phi}+2 \beta \dot{\phi}+\sin \phi+\frac{\omega^{2} \sin 2 \phi}{2} \sigma^{2}=\xi(t) .
$$

Here is taken into account of the fact that $\left\langle\zeta_{1}^{2}+\zeta_{2}^{2}\right\rangle=2 \sigma^{2}$. The Fokker-Planck equation for the probability density $w(\phi, \dot{\phi}, t)$ associated with Eq. (4.21) is conveniently written [26] as

$$
\frac{\partial w}{\partial t}=-\left[\dot{\phi} \frac{\partial w}{\partial \phi}-\left(\sin \phi+\frac{\omega^{2} \sigma^{2}}{2} \sin 2 \phi\right) \frac{\partial w}{\partial \dot{\phi}}\right]+\left[\frac{\partial(\dot{\phi} w)}{\partial \dot{\phi}}+\frac{\kappa}{2} \frac{\partial^{2} w}{\partial \dot{\phi}^{2}}\right] .
$$

As shown in [26], a steady-state solution of Eq. (4.22) can be found by equating each from the braces in the right-hand side to zero. As a result, we obtain

$$
w(\phi, \dot{\phi})=C \exp \left(\frac{\dot{\phi}^{2}}{\kappa}\right) w(\phi),
$$

where

$$
w(\phi)=C_{1} \exp \left[\frac{2}{\kappa}\left(\cos \phi+\frac{\omega^{2} \sigma^{2}}{4} \cos 2 \phi\right)\right],
$$


and $C$ and $C_{1}$ are normalization constants. It is easily seen from (4.23) that the function $w(\phi, \dot{\phi})$ has three extrema for $\dot{\phi}=0$ and $\phi=0, \phi=\pi, \phi=\arccos \left(-1 / \omega^{2} \sigma^{2}\right)$. The last of these exist only if $\omega^{2} \sigma^{2}>1$, i.e., it is induced by the random vibration of the pendulum suspension axis. Under this condition the probability density $w(\phi)$ has two maxima (for $\phi=0$ and $\phi=\pi$ ) and one minimum (for $\phi=\arccos \left(-1 / \omega^{2} \sigma^{2}\right)$ ). If $\omega^{2} \sigma^{2}<1$ than the probability density $w(\phi)$ has only one maximum (for $\phi=0$ ) and one minimum (for $\phi=\pi$ ). Thus, the random vibration considered causes multistability. An example of the transformation of the probability density $w(\phi)$ with increasing $\sigma^{2}$ is shown in Fig. 12 for $\kappa=4$.

\subsection{Noise-induced oscillations}

Here we consider noise-induced phase transitions revealing themselves in excitation of oscillations. It should be noted that there are different mechanisms of such excitation. We will discuss a selection of them.

4.2.1. Noise-induced oscillations in a pendulum with randomly vibrated suspension axis.

The influence of additive noise

Landa and Zaikin considered [8-10] a pendulum with a randomly vibrated suspension axis and nonlinear friction described by the equation

$$
\ddot{\varphi}+2 \beta\left(1+\alpha \dot{\varphi}^{2}\right) \dot{\varphi}+\omega_{0}^{2}\left(1+\xi_{1}(t)\right) \sin \varphi=\omega_{0}^{2} \xi_{2}(t),
$$

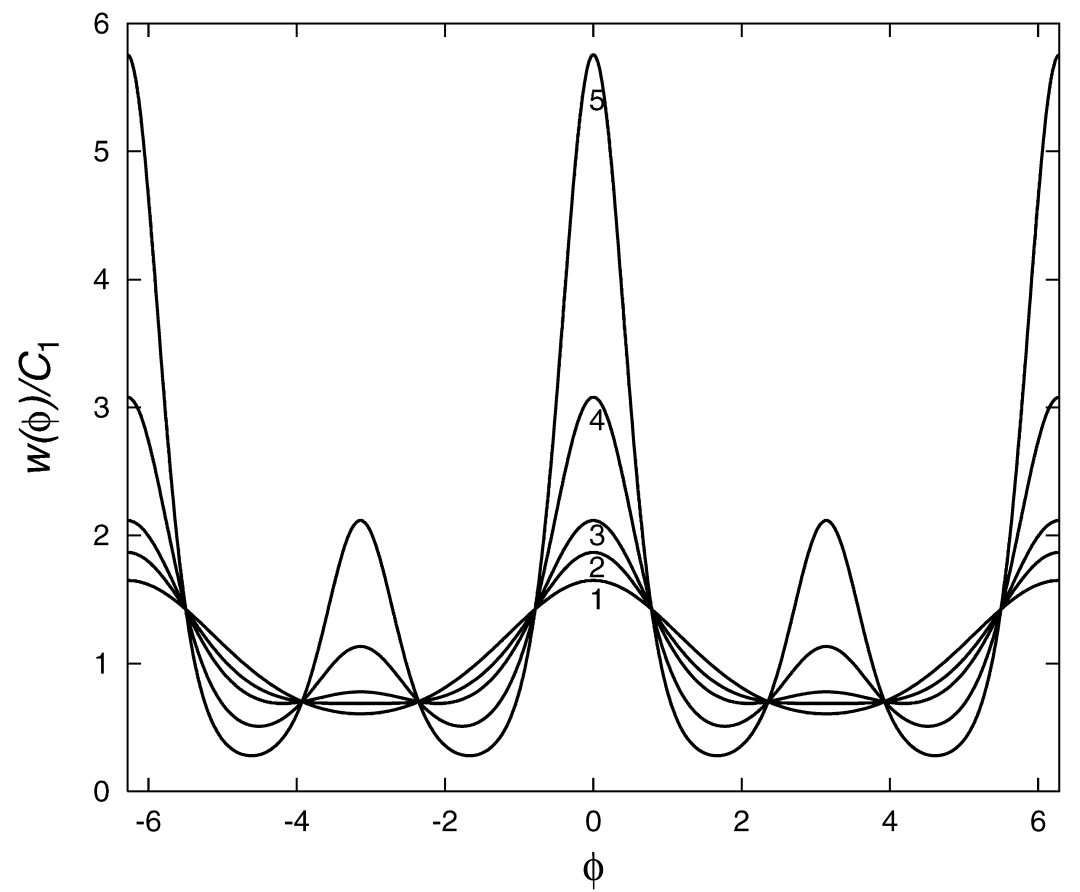

Fig. 12. Examples of the transformation of the function $w(\phi) / C_{1}$, determined by the expression (4.24), with increasing $\sigma^{2}$ for $\kappa=4$ and $\omega \sigma^{2}=0,1,2,5$ and 10 for curves $1-5$, respectively. 
where $\varphi$ is the pendulum's angular deviation from the equilibrium position, $\omega_{0}=\sqrt{\mathrm{mbg} / J}$ is the natural frequency of the pendulum's small free oscillations, $J$ and $m$ are the moment of inertia and the mass of the pendulum, $b$ is the distance between the center of mass and the suspension axis, $g$ is the acceleration due to gravity, $\beta=H / 2 J$ is the linear damping factor, $H \dot{\varphi}$ is the moment of the friction force in the linear approximation, $\alpha$ is the coefficient of nonlinear friction, $\xi_{1}(t)$ is a multiplicative noise proportional to the acceleration of the suspension axis, and $\xi_{2}(t)$ is an additive noise. $\xi_{1}(t)$ is assumed to be a comparatively wide-band random process with nonzero power spectrum density at the frequency $2 \omega_{0} . \xi_{2}(t)$ can be either uncorrelated with $\xi_{1}(t)$, or correlated if it has a component caused by a deviation away from the vertical in the direction of the suspension axis vibration.

We will assume that the suspension axis vibration is vertical, and moderately small in amplitude, so that pendulum oscillations can be considered small enough for $\varphi$ to be substituted in place of $\sin \varphi$ in Eq. (4.25).

An approximate analytical solution of the problem can be obtained on the assumptions that $\beta / \omega_{0} \sim \varepsilon, \xi_{1}(t) \sim \sqrt{\varepsilon}$, and $\xi_{2}(t) \sim \sqrt{\varepsilon}$, where $\varepsilon$ is a certain small parameter which should be put equal to unity in the final results. Eq. (4.25) can then be solved by the Krylov-Bogolubov method; to do this we set $\varphi=A(t) \cos \psi(t)+\varepsilon u_{1}+\cdots$, where $\psi(t)=\omega_{0} t+\phi(t)$,

$$
\dot{A}=\varepsilon f_{1}+\cdots, \quad \dot{\phi}=\varepsilon F_{1}+\cdots,
$$

$u_{1}, \ldots, f_{1}, \ldots, F_{1}, \ldots$, are unknown functions. By using the Krylov-Bogolubov technique for stochastic equations (see [26]) we find the expressions for the unknown functions $f_{1}$ and $F_{1}$. Substituting these expressions into Eqs. (4.26) we obtain

$$
\begin{aligned}
& \dot{A}=-\beta\left(1+\frac{3}{4} \alpha \omega_{0}^{2} A^{2}\right) A+\omega_{0} \overline{g_{1}\left(A, \psi(t), \xi_{1}(t), \xi_{2}(t)\right)}, \\
& \dot{\phi}=\omega_{0} \overline{g_{2}\left(A, \psi(t), \xi_{1}(t), \xi_{2}(t)\right)},
\end{aligned}
$$

where

$$
\begin{aligned}
& g_{1}(A, \phi, t)=\frac{A}{2} \xi_{1}(t) \sin 2 \psi(t)-\xi_{2}(t) \sin \psi(t), \\
& g_{2}(A, \phi, t)=\xi_{1}(t) \cos ^{2} \psi(t)-\frac{1}{A} \xi_{2}(t) \cos \psi(t),
\end{aligned}
$$

the bar over the expression signify averaging over time. As follows from [26], the Fokker-Planck equation associated with Eqs. (4.27) and (4.28) is

$$
\begin{aligned}
\frac{\partial w(A, \phi, t)}{\partial t}= & -\frac{\partial}{\partial A}\left[\left(-\beta\left(1+\frac{3}{4} \alpha \omega_{0}^{2} A^{2}\right) A+\omega_{0}^{2} R_{1}\right) w(A, \phi, t)\right]-\omega_{0}^{2} R_{2} \frac{\partial w(A, \phi, t)}{\partial \phi} \\
& +\frac{\omega_{0}^{2}}{2}\left\{\frac{\partial^{2}}{\partial A^{2}}\left(\left(\frac{K_{11}}{4} A^{2}+K_{12}\right) w(A, \phi, t)\right)+\left(K_{21}+\frac{K_{22}}{A^{2}}\right) \frac{\partial^{2} w(A, \phi, t)}{\partial \phi^{2}}\right\},
\end{aligned}
$$

where

$$
\left.\left.R_{1}=\int_{-\infty}^{0}\left(\overline{\left\langle\frac{\partial g_{1}(A, \phi, t)}{\partial A} g_{1}(A, \phi, t+\tau)\right.}\right\rangle+\overline{\left\langle\frac{\partial g_{1}(A, \phi, t)}{\partial \phi} g_{2}(A, \phi, t+\tau)\right.}\right\rangle\right) \mathrm{d} \tau,
$$




$$
\left.R_{2}=\int_{-\infty}^{0}\left(\left\langle\overline{\frac{\partial g_{2}(A, \phi, t)}{\partial A} g_{1}(A, \phi, t+\tau)}\right\rangle+\overline{\frac{\partial g_{2}(A, \phi, t)}{\partial \phi} g_{2}(A, \phi, t+\tau)}\right\rangle\right) \mathrm{d} \tau,
$$

the angular brackets signify averaging over the statistical ensemble,

$$
\begin{aligned}
& K_{11}=\frac{1}{2} \kappa_{\xi_{1}}\left(2 \omega_{0}\right), \quad K_{12}=\frac{1}{2} \kappa_{\xi_{2}}\left(\omega_{0}\right), \\
& K_{21}=\frac{1}{4}\left(\kappa_{\xi_{1}}(0)+\frac{1}{2} \kappa_{\xi_{1}}\left(2 \omega_{0}\right)\right), \quad K_{22}=\frac{1}{4}\left(\kappa_{\xi_{2}}(0)+\frac{1}{2} \kappa_{\xi_{2}}\left(\omega_{0}\right)\right),
\end{aligned}
$$

and

$$
\kappa_{\xi}(\omega)=\int_{-\infty}^{\infty}\langle\xi(t) \xi(t+\tau)\rangle \cos \omega \tau \mathrm{d} \tau
$$

is the power spectrum density of the process $\xi(t)$ at the frequency $\omega$. Let us now calculate the integrals (4.30) and (4.31) taking into account the expressions for $g_{1}$ and $g_{2}$. As a result we obtain

$$
\begin{aligned}
R_{1} & =\frac{3 A}{8} \int_{-\infty}^{0}\left\langle\xi_{1}(t) \xi_{1}(t+\tau)\right\rangle \cos 2 \omega_{0} \tau \mathrm{d} \tau+\frac{1}{2 A} \int_{-\infty}^{0}\left\langle\xi_{2}(t) \xi_{2}(t+\tau)\right\rangle \cos \omega_{0} \tau \mathrm{d} \tau \\
& =\frac{3 K_{11}}{8} A+\frac{K_{12}}{2 A}, \\
R_{2} & =\frac{1}{4} \int_{-\infty}^{0}\left\langle\xi_{1}(t) \xi_{1}(t+\tau)\right\rangle \sin 2 \omega_{0} \tau \mathrm{d} \tau-\frac{1}{A^{2}} \int_{-\infty}^{0}\left\langle\xi_{2}(t) \xi_{2}(t+\tau)\right\rangle \sin \omega_{0} \tau \mathrm{d} \tau \equiv M .
\end{aligned}
$$

The value of $M$ depends on the characteristics of the random processes $\xi_{1}(t)$ and $\xi_{2}(t)$ : if they are white noises then $M=0$; but if, for example, $\xi_{2}(t)$ is white noise but $\xi_{1}(t)$ has a finite correlation time and its power spectrum density is

$$
\kappa_{\xi_{1}}(\omega)=\frac{a_{1}^{2} \kappa_{\xi_{1}}\left(2 \omega_{0}\right)}{\left(\omega-2 \omega_{0}\right)^{2}+a_{1}^{2}},
$$

then

$$
M=-\frac{a_{1} \omega_{0} \kappa_{\xi_{1}}\left(2 \omega_{0}\right)}{4\left(16 \omega_{0}^{2}+a_{1}^{2}\right)} .
$$

It should be noted that $M$ is negative, resulting in a decrease of the mean oscillation frequency. This decrease is the more considerable the larger is the intensity of the noise.

The following Langevin equations can be related to the Fokker-Planck equation (4.29) in view of (4.34) and(4.35):

$$
\begin{aligned}
& \dot{A}=\beta\left(\eta-\frac{3 \omega_{0}^{2}}{4} \alpha A^{2}\right) A+\frac{\omega_{0}^{2}}{2 A} K_{12}+\frac{\omega_{0}}{2} A \zeta_{11}(t)+\omega_{0} \zeta_{12}(t), \\
& \dot{\phi}=\omega_{0}^{2} M+\omega_{0}\left(\zeta_{21}(t)+\frac{\zeta_{22}(t)}{A}\right),
\end{aligned}
$$

where $\eta=3 \omega_{0}^{2} K_{11} / 8 \beta-1$, and $\zeta_{11}(t), \zeta_{12}(t), \zeta_{21}(t)$, and $\zeta_{22}(t)$ are each white noises of zero mean value uncorrelated with $A$. The intensities of these noises are $K_{11}, K_{12}, K_{21}$, and $K_{22}$, respectively. 
First we consider the case when additive noise is absent. We note that even in this case Eqs. (4.36) differ by numerical coefficient of $\eta$ from that derived in $[95,26]$. The reason is that authors, using the variable $u=\ln A$ in place of $A$, implicitly ignored correlation between the noise $\xi(t)$ and the amplitude $A$. The same mistake is reproduced in $[1,8,9]$.

For $\kappa_{2} \equiv 0$ the steady-state solution of Eq. (4.29), satisfying the condition of zero probability flux, is

$$
w(A, \phi)=\frac{C}{2 \pi A^{2}} \exp \left\{\frac{3}{1+\eta}\left(\eta \ln A-\frac{a A^{2}}{2}\right)\right\},
$$

where $a=3 \alpha \omega_{0}^{2} / 4$ is the nonlinear parameter. The constant $C$ is determined from the normalization condition

$$
\int_{0}^{2 \pi} \int_{0}^{\infty} w(A, \phi) A \mathrm{~d} A \mathrm{~d} \phi=1 .
$$

Upon integrating (4.37) over $\phi$ we find the expression for the probability density of the amplitude of oscillations

$$
w(A)=C A^{(2 \eta-1) /(1+\eta)} \exp \left(-\frac{3 a A^{2}}{2(1+\eta)}\right) .
$$

From the normalization condition we find

$$
C=2\left\{\begin{array}{cc}
\left(\frac{3 a}{2(1+\eta)}\right)^{3 \eta / 2(1+\eta)} \frac{1}{\Gamma(3 \eta / 2(1+\eta))} & \text { for } \eta \geq 0 \\
0 & \text { for } \eta \leq 0
\end{array}\right.
$$

Hence,

$$
w(A)=2\left\{\begin{array}{cl}
\left(\frac{3 a}{2(1+\eta)}\right)^{3 \eta / 2(1+\eta)} \frac{A^{(2 \eta-1) /(1+\eta)}}{\Gamma(3 \eta / 2(1+\eta))} \exp \left(-\frac{3 a A^{2}}{2(1+\eta)}\right) & \text { for } \eta \geq 0, \\
\delta(A) & \text { for } \eta \leq 0 .
\end{array}\right.
$$

The fact that for $\eta \leq 0$ the probability density of the amplitude turns out to be a $\delta$-function is associated with the absence of additive noise (see below).

Using (4.40) we can find $\langle A\rangle$ and $\left\langle A^{2}\right\rangle$ :

$$
\begin{aligned}
& \langle A\rangle=\left\{\begin{array}{cc}
\sqrt{\frac{3}{2 a(1+\eta)}} \frac{\Gamma((4 \eta+1) / 2(1+\eta))}{\Gamma(3 \eta / 2(1+\eta)+1)} \eta & \text { for } \eta \geq 0, \\
0 & \text { for } \eta \leq 0,
\end{array}\right. \\
& \left\langle A^{2}\right\rangle=\left\{\begin{array}{cl}
\eta / a & \text { for } \eta \geq 0, \\
0 & \text { for } \eta \leq 0 .
\end{array}\right.
\end{aligned}
$$

It is therefore evident that for $\eta>0$ parametric excitation of pendulum oscillations occurs under the influence of multiplicative noise. This manifests itself in the fact that the mean values of the amplitude and of the amplitude-squared become nonzero. The availability of this parametric 
excitations implies a transition of the system to a new state, which is to say that a phase transition has occurred in the system. Thus, the condition $\eta=0$ gives the onset of the phase transition. It follows that, in the absence of additive noise, the critical value of the multiplicative noise intensity is

$$
\kappa_{\xi}^{\mathrm{cr}}\left(2 \omega_{0}\right) \equiv \kappa_{\mathrm{cr}}=16 \beta / 3 \omega_{0}^{2} .
$$

The parameter $\eta$ characterizes the extent to which the intensity of multiplicative noise component exceeds its critical value.

It should be noted that, for $\eta>0$, the steady state $A=0$ loses its stability and the state $A \neq 0$ becomes stable. At the same time, (4.40) implies that the probability density of $A^{2}$ is monotonically decreasing with increasing $A^{2}$ for any values of $\eta>0$ (see Fig. 13). The same result has also been obtained by numerical simulation. Hence, the appearance of a new stable state need not be accompanied by the appearance of a new maximum in the probability distribution.

In the case when the intensity of additive noise is nonzero, the steady-state solution of Eq. (4.29), satisfying the condition of zero probability flux, is conveniently written as

$$
w(A, \phi)=\frac{C a}{2 \pi\left(a A^{2}+q\right)} \exp \left\{\int \frac{3\left(\eta-a A^{2}\right) a A^{2}+q}{(1+\eta)\left(a A^{2}+q\right) A} \mathrm{~d} A\right\},
$$

where $q=4 a K_{12} / K_{11}$ characterizes the ratio between the intensities of additive and multiplicative noises.

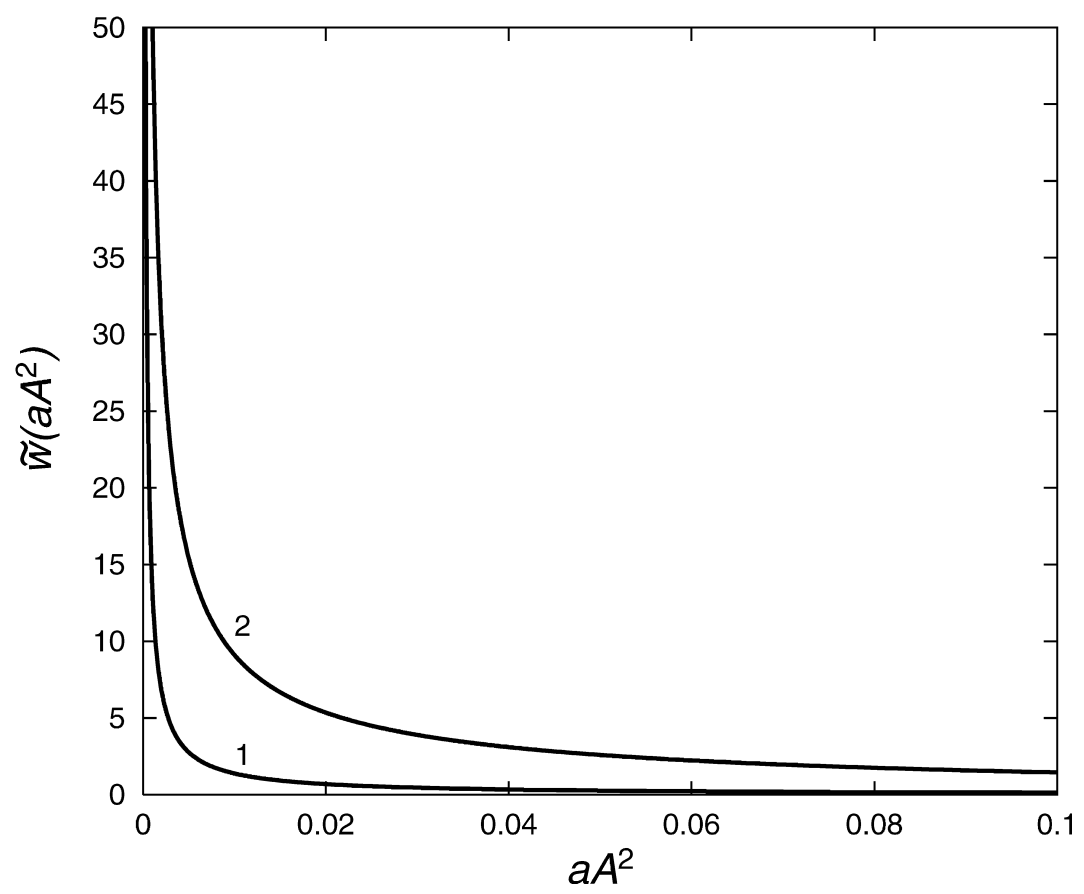

Fig. 13. Plots of $\tilde{w}\left(a A^{2}\right)=w(A) / 2 a A$ for $\eta=0.01$ (curve 1), and $\eta=0.2$ (curve 2). 
Upon integrating (4.44) over $\phi$ and calculating the integral under the exponential symbol, we obtain

$$
w(A)=2 \pi A w(A, \phi)=C A^{2}\left(A^{2}+q / a\right)^{3(q-1) / 2(1+\eta)} \exp \left(-\frac{3 a A^{2}}{2(1+\eta)}\right) .
$$

It follows from the normalization condition that

$$
C^{-1}=\int_{0}^{\infty} A^{2}\left(A^{2}+q / a\right)^{3(q-1) / 2(1+\eta)} \exp \left(-\frac{3 a A^{2}}{2(1+\eta)}\right) \mathrm{d} A .
$$

The integral on the right-hand side of (4.46) can be expressed in terms of a Whittaker function [96]. As a result we find

$$
C^{-1}=\frac{\sqrt{\pi}}{4 a^{2 \mu} q^{1 / 2-\mu}}\left(\frac{3}{2(1+\eta)}\right)^{-\mu-1 / 2} \exp \left(\frac{3 q}{4(1+\eta)}\right) W_{\mu-1, \mu}\left(\frac{3 q}{2(1+\eta)}\right),
$$

where $\mu=3(\eta+q) / 4(1+\eta)$. We can obtain an expression for $C$ in explicit form in the limiting case when the additive noise intensity is small compared to that of the multiplicative noise, so that

$$
q \ll 1 \text {. }
$$

In this case we can use a representation of the Whittaker function $W_{\lambda, \mu}(z)$ in terms of two other Whittaker functions $M_{\lambda, \mu}(z)$ and $M_{\lambda,-\mu}(z)$ [96]:

$$
W_{\lambda, \mu}(z)=\frac{\Gamma(-2 \mu)}{\Gamma(1 / 2-\mu-\lambda)} M_{\lambda, \mu}(z)+\frac{\Gamma(2 \mu)}{\Gamma(1 / 2+\mu-\lambda)} M_{\lambda,-\mu}(z) .
$$

We then expand each of the functions $M_{\lambda, \mu}(z)$ and $M_{\lambda,-\mu}(z)$ in powers of $z$ [96]:

$$
\begin{aligned}
W_{\lambda, \mu}(z)= & \sqrt{z} \exp \left(-\frac{z}{2}\right)\left[\frac{\Gamma(-2 \mu)}{\Gamma(1 / 2-\mu-\lambda)} z^{\mu}\left(1+\frac{1-2(\lambda-\mu)}{2(1+2 \mu)} z+\cdots\right)\right. \\
& \left.+\frac{\Gamma(2 \mu)}{\Gamma(1 / 2+\mu-\lambda)} z^{-\mu}\left(1+\frac{1-2(\lambda+\mu)}{2(1-2 \mu)} z+\cdots\right)\right] .
\end{aligned}
$$

Substituting (4.50) into (4.47) we obtain

$$
\begin{aligned}
C^{-1}= & \frac{\sqrt{\pi}}{4 a^{2 \mu}}\left[\frac{\Gamma(-2 \mu)}{\Gamma(3 / 2-2 \mu)} q^{2 \mu}\left(1+\frac{9 q}{4(1+2 \mu)(1+\eta)}+\cdots\right)\right. \\
& \left.+\frac{\Gamma(2 \mu)}{\Gamma(3 / 2)}\left(\frac{2(1+\eta)}{3}\right)^{2 \mu}\left(1+\frac{3(3-4 \mu) q}{4(1-2 \mu)(1+\eta)}+\cdots\right)\right] .
\end{aligned}
$$

The expression (4.39), obtained in the absence of additive noise, follows at once from (4.51) for $q \rightarrow 0$.

The probability distribution (4.45) for $q \neq 0$ differs essentially from (4.40): first, it is not a $\delta$-function for $\eta<0$ and, secondly, $w(A)=0$ for $A=0$. Plots of $\tilde{w}\left(a A^{2}\right)=w(A) / 2 a A$ for $q_{0}=q(1+\eta)=3 a \omega_{0}^{2} K_{12} / 2 \beta=0.01$ and different values of $\eta$ are shown in Fig. 14. 


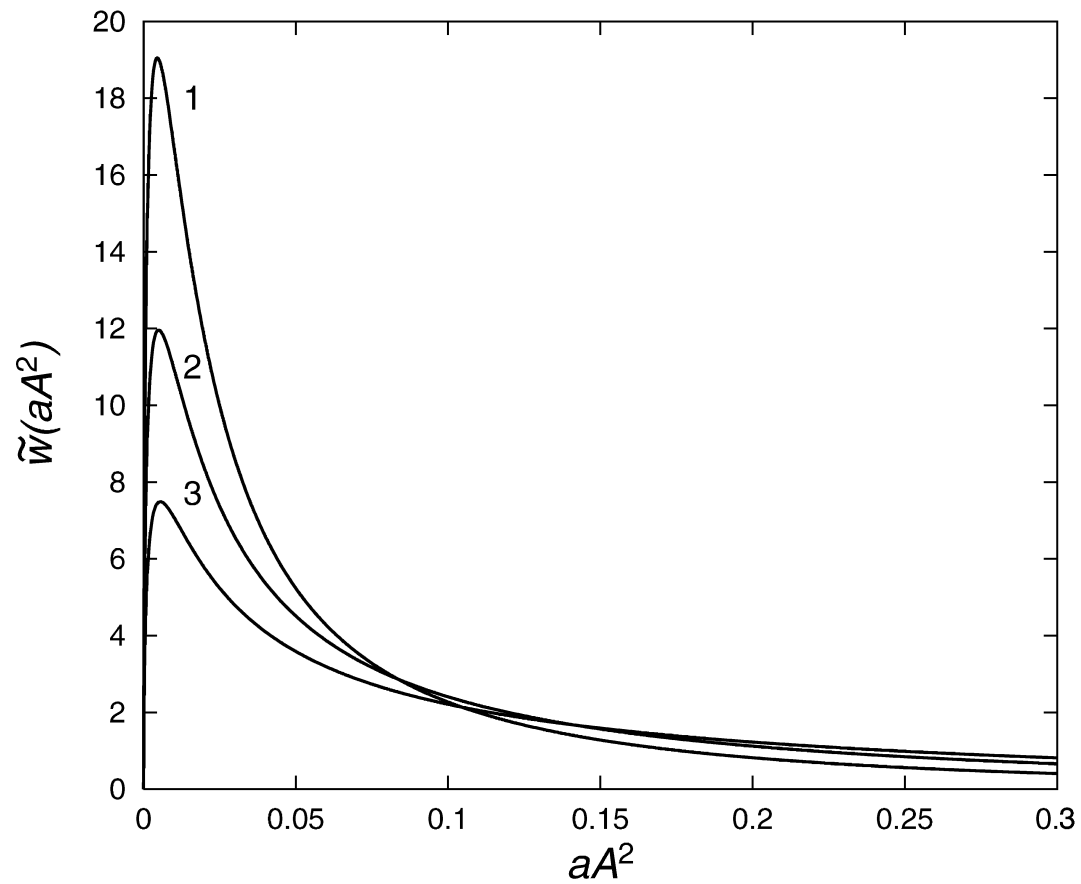

Fig. 14. Plots of $\tilde{w}\left(a A^{2}\right)=w(A) / 2 a A$ for $q=0.01 /(1+\eta)$ and $\eta=-0.2,0$ and 0.2 for curves $1-3$, respectively.

Using (4.45) and (4.47) we can calculate $\langle A\rangle$ and $\left\langle A^{2}\right\rangle$. For example, for $\left\langle A^{2}\right\rangle$ we obtain

$$
a\left\langle A^{2}\right\rangle=\sqrt{\frac{3 q(1+\eta)}{2}} \frac{W_{\mu-3 / 2, \mu+1 / 2}(3 q / 2(1+\eta))}{W_{\mu-1, \mu}(3 q / 2(1+\eta))} .
$$

Taking into account the following recursion relation [96]

$$
W_{\lambda, \mu}(z)=\sqrt{z} W_{\lambda-1 / 2, \mu+1 / 2}(z)+\left(\frac{1}{2}-\lambda-\mu\right) W_{\lambda-1, \mu}(z),
$$

expression (4.52) can be rewritten as

$$
a\left\langle A^{2}\right\rangle=(1+\eta)\left(1-\left(\frac{3}{2}-2 \mu\right) \frac{W_{\mu-2, \mu}(3 q / 2(1+\eta))}{W_{\mu-1, \mu}(3 q / 2(1+\eta))}\right) .
$$

The expression for $\left\langle A^{2}\right\rangle$ can be obtained in explicit form only with the constraint (4.48). Using (4.50) we find for $W_{\mu-2, \mu}(z) / W_{\mu-1, \mu}(z)$ the following approximate expression:

$$
\begin{aligned}
\frac{W_{\mu-2, \mu}(z)}{W_{\mu-1, \mu}(z)} \approx & \frac{2}{(3-4 \mu)}\left[\frac{\sqrt{\pi}}{2} \Gamma(-2 \mu) z^{\mu}(1-2 \mu)(2(1+2 \mu)+5 z)\right. \\
& \left.+\Gamma(2 \mu) \Gamma\left(\frac{3}{2}-2 \mu\right)\left(1-\frac{4 \mu}{3}\right) z^{-\mu}(1+2 \mu)(2(1-2 \mu)+(5-4 \mu) z)\right]
\end{aligned}
$$




$$
\begin{aligned}
& \times\left[\frac{\sqrt{\pi}}{2} \Gamma(-2 \mu) z^{\mu}(1-2 \mu)(2(1+2 \mu)+3 z)\right. \\
& \left.+\Gamma(2 \mu) \Gamma\left(\frac{3}{2}-2 \mu\right) z^{-\mu}(1+2 \mu)(2(1-2 \mu)+(3-4 \mu) z)\right]^{-1} .
\end{aligned}
$$

Substituting (4.54) in (4.53) we obtain

$$
\begin{aligned}
a\left\langle A^{2}\right\rangle \approx & (1+\eta)\left[\frac{4 \mu}{3} \Gamma(2 \mu) \Gamma\left(\frac{3}{2}-2 \mu\right)(1+2 \mu)\left(2(1-2 \mu)+(5-4 \mu) \frac{3 q}{2(1+\eta)}\right)\right. \\
& \left.-\frac{3 q}{2(1+\eta)}\left(\sqrt{\pi} \Gamma(-2 \mu)(1-2 \mu)\left(\frac{3 q}{2(1+\eta)}\right)^{2 \mu}+2 \Gamma(2 \mu) \Gamma\left(\frac{3}{2}-2 \mu\right)(1+2 \mu)\right)\right] \\
& \times\left[\frac{\sqrt{\pi}}{2} \Gamma(-2 \mu)(1-2 \mu)\left(\frac{3 q}{2(1+\eta)}\right)^{2 \mu}\left(2(1+2 \mu)+\frac{9 q}{2(1+\eta)}\right)\right. \\
& \left.+\Gamma(2 \mu) \Gamma\left(\frac{3}{2}-2 \mu\right)(1+2 \mu)\left(2(1-2 \mu)+\frac{3(3-4 \mu) q}{2(1+\eta)}\right)\right]^{-1} .
\end{aligned}
$$

The corresponding dependence of $a\left\langle A^{2}\right\rangle$ on $\eta$ for different values of the parameter $q_{0}$ is illustrated in Fig. 15. We see that slight additive noise results in a smoothing of the dependences of the mean

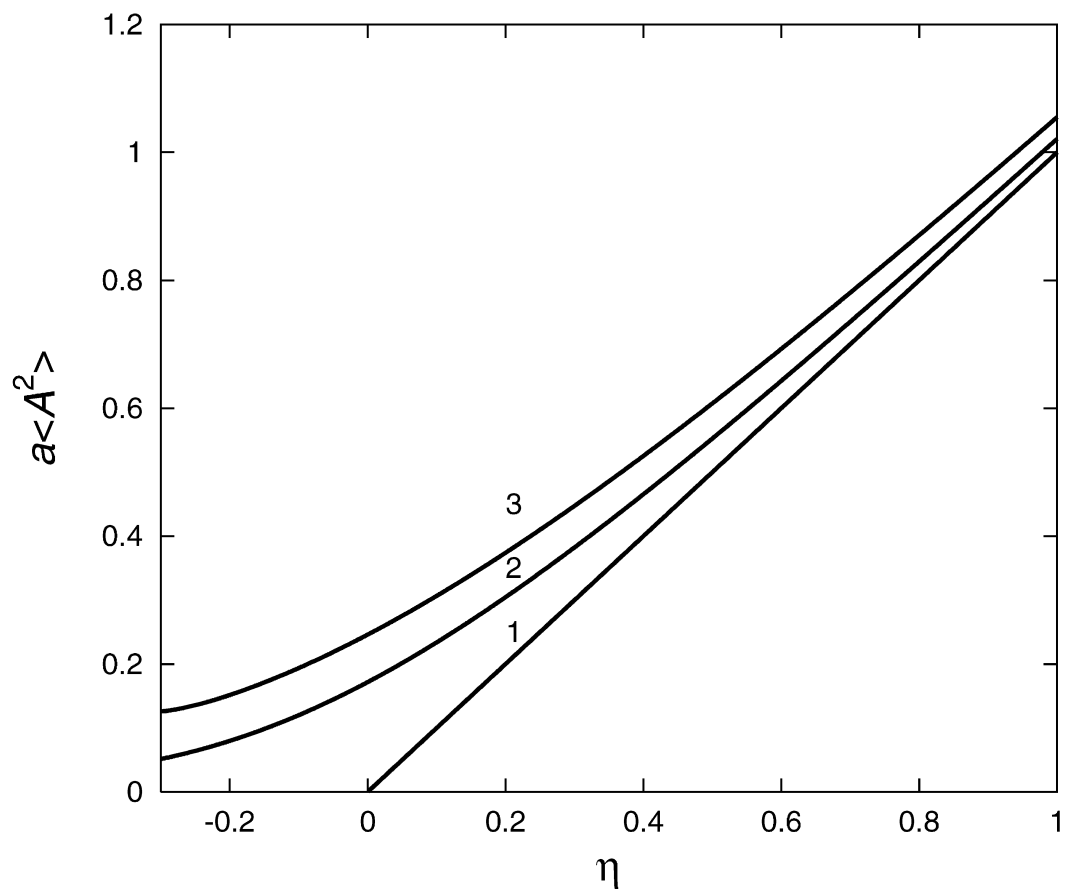

Fig. 15. The dependence of $a\left\langle A^{2}\right\rangle$ on $\eta$ for $q_{0}=0,0.005$ and 0.02 for curves $1-3$, respectively. 
oscillation amplitude and amplitude-squared on the multiplicative noise intensity: they lose the discontinuities inherent in the phase transition induced by multiplicative noise alone.

\subsubsection{Phase transition in an oscillator with quadratic nonlinearity induced by additive noise}

We now show that in an oscillator with a quadratic nonlinearity in the restoring force, additive noise can result in a nonequilibrium phase transition similar to that considered above. The physical mechanism of such the transition is subharmonic resonance [1].

Let us consider a nonlinear oscillator described by the following equation:

$$
\ddot{x}+2 \beta \dot{x}+\omega_{0}^{2}\left(1+b x+\gamma b^{2} x^{2}\right) x=\omega_{0}^{2} \xi(t),
$$

where $\beta$ is the friction factor, $\xi(t)$ is a sufficiently wide-band random process with zero mean value, $b$ is the quadratic nonlinearity parameter, and the term $\gamma b^{2} x^{3}$ is introduced to prevent the solution diverging to infinity. ${ }^{8}$ Substituting $x^{\prime}=b x$ into Eq. (4.56) and dropping primes we obtain

$$
\ddot{x}+2 \beta \dot{x}+\omega_{0}^{2}\left(1+x+\gamma x^{2}\right) x=\omega_{0}^{2} b \xi(t) .
$$

So, variation of the nonlinearity parameter $b$ is evidently equivalent to variation of the noise intensity.

Because the noise intensity which is necessary to induce the phase transition in question is not small, we cannot use the Krylov-Bogolyubov method for Eq. (4.57) directly. To use this method, we have to introduce new variables (just as for calculating subharmonic resonances [1]). So, we substitute into Eq. (4.57)

$$
x(t)=y(t)+\chi(t),
$$

where $\chi(t)$ is a random process satisfying the equation

$$
\ddot{\chi}+2 \beta \dot{\chi}+\omega_{0}^{2} \chi=\omega_{0}^{2} b \xi(t) .
$$

In this way we can find the equation for the variable $y(t)$. It can conveniently be written as

$$
\ddot{y}+2 \beta \dot{y}+\omega_{0}^{2}\left(1+y+2 \chi+\gamma\left(3 \chi^{2}+y^{2}+3 y \chi\right)\right) y=\xi_{1}(t),
$$

where $\xi_{1}(t)=-\omega_{0}^{2} \chi^{2}(t)(1+\gamma \chi(t))$ is additive noise. The terms $2 \chi y$ and $3 \gamma \chi^{2} y$ are responsible for the phase transition, whereas the terms $\gamma y^{3}$ and $\gamma \chi y^{2}$ are responsible for limiting the oscillation amplitude.

An approximate analytic solution of Eq. (4.60), consistent with Eq. (4.59), is possible in the specific case when the spectral density of the random process $\xi(t)$ at the main frequency $\omega_{0}$ is negligibly small, whereas it is sufficiently large at the frequency $2 \omega_{0}$. The random force in Eq. (4.59) is then nonresonant. Consequently, $\chi(t)$ is sufficiently small that we can ignore in Eq. (4.60) the two terms $\xi_{1}(t)$ and $3 \gamma \chi^{2} y$. As a result we obtain the following approximate equation for $y$ :

$$
\ddot{y}+2 \beta \dot{y}+\omega_{0}^{2}\left(1+y+2 \chi+\gamma y^{2}\right) y=-3 \omega_{0}^{2} \gamma y^{2} \chi .
$$

\footnotetext{
${ }^{8}$ This divergence is caused by the presence of an unstable singular point of Eq. (4.56) for $\gamma<0.25$.
} 
Putting $y=A(t) \cos \psi(t)+\cdots$, where $\psi(t)=\omega_{0} t+\phi(t)$ and using the Krylov-Bogolyubov method for stochastic equations, we obtain the following truncated equations for $A(t)$ and $\phi(t)$ :

$$
\dot{A}=-\beta\left(1+a A^{2}\right) A+\omega_{0} \overline{g_{1}(A, \phi, t)}, \quad \dot{\phi}=\omega_{0} \overline{g_{2}(A, \phi, t)},
$$

where $a=(3 / 4) \gamma$,

$$
\begin{aligned}
& g_{1}(A, \phi, t)=A \chi(t) \sin 2 \psi(t)+\frac{3 \gamma A^{2}}{2} \chi(t) \cos \psi(t) \sin 2 \psi(t), \\
& g_{2}(A, \phi, t)=2 \chi(t) \cos ^{2} \psi(t)+3 \gamma A \chi(t) \cos ^{3} \psi(t) .
\end{aligned}
$$

Substituting (4.63) into (4.30) and (4.31) we find

$$
\begin{aligned}
& R_{1}=\frac{3 K_{1}}{2} A+\frac{45 \gamma^{2}}{32}\left(K_{2}+K_{3}\right) A^{3}, \\
& R_{2}=\int_{-\infty}^{0}\langle\chi(t) \chi(t+\tau)\rangle\left(\sin 2 \omega_{0} \tau+\frac{9 \gamma^{2} A^{3}}{4}\left(3 \sin \omega_{0} \tau+\sin 3 \omega_{0} \tau\right)\right) \mathrm{d} \tau \equiv M_{1},
\end{aligned}
$$

where $K_{1}=\kappa_{\chi}\left(2 \omega_{0}\right) / 2, K_{2}=\kappa_{\chi}\left(\omega_{0}\right) / 2, K_{3}=\kappa_{\chi}\left(3 \omega_{0}\right) / 2$. Taking into account of (4.64) and(4.65) we can write the Fokker-Planck equation associated with Eqs. (4.62) in the following form:

$$
\begin{aligned}
\frac{\partial w(A, \phi, t)}{\partial t}= & -\frac{\partial}{\partial A}\left(\beta\left(\eta-a_{1} A^{2}\right) A w(A, \phi, t)\right)-\omega_{0}^{2} M_{1} \frac{\partial w(A, \phi, t)}{\partial \phi} \\
& +\frac{\omega_{0}^{2}}{2}\left\{\frac{\partial^{2}}{\partial A^{2}}\left[\left(K_{1}+\frac{9 \gamma^{2} A^{2}}{16}\left(K_{2}+K_{3}\right)\right) A^{2} w(A, \phi, t)\right]\right. \\
& \left.+\left(2 K_{0}+K_{1}+\frac{9 \gamma^{2} A^{2}}{16}\left(K_{2}+K_{3}\right)\right) \frac{\partial^{2} w(A, \phi, t)}{\partial \phi^{2}}\right\},
\end{aligned}
$$

where

$$
\begin{aligned}
& \eta=\frac{3 \omega_{0}^{2} K_{1}}{2 \beta}-1, \\
& a_{1}=a-\frac{5}{3} r(1+\eta), \quad r=\frac{9 \gamma^{2}}{16} \frac{K_{2}+K_{3}}{K_{1}}, \quad K_{0}=\kappa_{\chi}(0) .
\end{aligned}
$$

The steady-state solution of Eq. (4.66), satisfying the condition for zero probability flux, is

$$
\begin{aligned}
w(A, \phi) & =\frac{C}{2 \pi A^{2}\left(1+r A^{2}\right)} \exp \left(\int \frac{2 \beta\left(\eta-a_{1} A^{2}\right)}{\omega_{0}^{2} K_{1} A\left(1+r A^{2}\right)} \mathrm{d} A\right) \\
& =\frac{C}{2 \pi A^{2}\left(1+r A^{2}\right)} \exp \left(\frac{3}{1+\eta} \int \frac{\left(\eta-a_{1} A^{2}\right)}{\left(1+r A^{2}\right) A} \mathrm{~d} A\right) .
\end{aligned}
$$

Upon integrating (4.68) over $\phi$ and calculating the integral under the exponential symbol we obtain

$$
w(A)=C A^{(2 \eta-1) /(1+\eta)}\left(1+r A^{2}\right)^{-\left((2+5 \eta) r+3 a_{1}\right) / 2 r(1+\eta)} .
$$


It is easily shown that for $r \rightarrow 0$ expression (4.69) is equivalent to (4.38). From the normalization condition we find

$$
C=2\left\{\begin{array}{cc}
\frac{a_{1}+r \eta}{a_{1}} r^{3 \eta / 2(1+\eta) \frac{\Gamma\left(3\left(a_{1}+r \eta\right) / 2 r(1+\eta)\right)}{\Gamma(3 \eta / 2(1+\eta)) \Gamma\left(3 a_{1} / 2 r(1+\eta)\right)}} & \text { for } \eta \geq 0, \\
0 & \text { for } \eta \leq 0 .
\end{array}\right.
$$

It then follows that the probability density of the amplitude is $\delta$-function for $\eta \leq 0$, as for the pendulum considered above. Using (4.69) and (4.70) we find $\left\langle A^{2}\right\rangle$ :

$$
\left\langle A^{2}\right\rangle=\frac{3 \eta}{3 a_{1}+r(2+5 \eta)}=\frac{\eta}{a-r} .
$$

We see that for $r \rightarrow 0$ expression (4.71) transforms to (4.42). If $r \neq 0$, the slope of the dependence $\left\langle A^{2}\right\rangle(\eta)$ increases with increasing $r$. It is evident that the solution found is valid only for $r<a$.

The results of numerical simulation of exact equations (4.59) and (4.60) in the case of sufficiently wide-band noise are shown in Fig. 16. For comparison, the results of numerical simulation of Eqs. (4.59) and (4.60) with $\xi_{1}(t) \equiv 0$ are given in the same figure. ${ }^{9}$ We see that in the first case the phase transition is extremely noisy. In the second case the phase transition is clearly defined: close to the critical point, the dependence of the variance of the variable $y_{\mathrm{r}}$ (which can be treated as an order parameter) on the parameter $b^{2}$ (which can be treated as temperature) can be approximated by the straight line described by the equation $\sigma_{y_{\mathrm{r}}}^{2}=0.056\left(b^{2}-b_{\mathrm{cr}}^{2}\right)$, where $b_{\mathrm{cr}} \approx 4.1$, i.e., the critical index is equal to 1 (see Fig. 16(a)).

Fig. 16(b) demonstrates that we can use as an order parameter not only the variance, but the mean value of the variable $y$ as well. Close to the critical point, the dependence of $\left\langle y_{\mathrm{r}}\right\rangle$ on $b^{2}$ can be approximated by the straight line $\left\langle y_{\mathrm{r}}\right\rangle=-0.025\left(b^{2}-b_{\mathrm{cr}}^{2}\right)$.

It should be noted that the phase transition would occur for a smaller value of $b^{2}$ if the term $3 \gamma \chi^{2} y$ in the reduced equation were ignored. Hence this term has the effect of suppressing the phase transition. This is also attested to by the fact that the slope of the dependence of $\sigma_{y \mathrm{r}}^{2}$ on $b^{2}$ decreases with increasing $b^{2}$ rather than increases.

To reduce the noise spectral density at the frequency $\omega_{0}$, we have passed our noise $\xi(t)$ through a bandpass filter with a central frequency of $2 \omega_{0}$ and a bandwidth of $\omega_{0}$. The spectral density of this noise is shown in Fig. 17. We see that it is indeed very small at the frequencies $\omega_{0}$ and $3 \omega_{0}$. Next we simulate Eqs. (4.59) and (4.60) using the filtered noise as $\xi(t)$. For comparison we simultaneously simulate Eq. (4.60) with $\xi_{1}(t) \equiv 0$. The results are shown in Fig. 18. We see that, even though the spectral density of the filtered noise $\xi(t)$ at the frequency $\omega_{0}$ is very small, the influence of the noise $\xi_{1}(t)$ and of the term $3 \gamma \chi^{2} y$ is considerable. The reason is that the component of the noise $\chi(t)$ at the frequency $\omega_{0}$ does not appear to be small, because it is resonant. Nevertheless, it can be seen that in this case the phase transition occurs for a somewhat smaller value of $b^{2}$ and the slope of the dependence of $\sigma_{\mathrm{r}}^{2}$ on $b^{2}$ in the vicinity of the transition is greater than in the case of wide-band noise.

\footnotetext{
${ }^{9}$ Eq. (4.60) for $\xi_{1}(t) \equiv 0$ we will call reduced equation and its solution we will denote $y_{\mathrm{r}}$.
} 

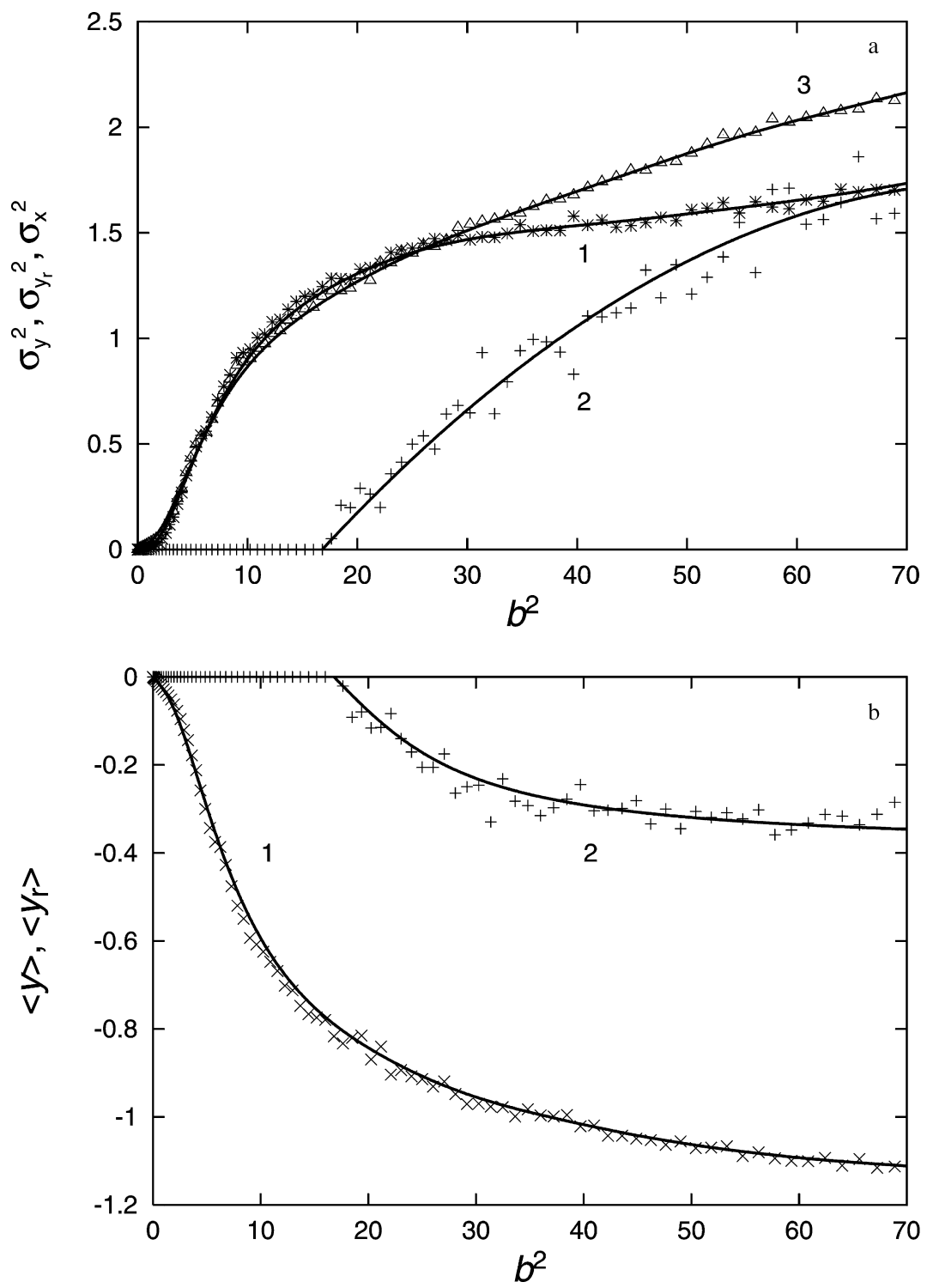

Fig. 16. (a) The numerical dependences of $\sigma_{y}^{2}$ (curve 1), $\sigma_{y_{\mathrm{r}}}^{2}$ (curve 2), and $\sigma_{x}^{2}$ (curve 3) on $b^{2}$ for $\gamma=0.251$. (b) The numerical dependences of $\langle y\rangle$ (curve 1) and $\left\langle y_{\mathrm{r}}\right\rangle$ (curve 2) on $b^{2}$ for same value of $\gamma$.

4.2.3. Oscillations in a standard model for childhood epidemics induced by random seasonal variations in the rate at which susceptible children contact infection

It is known that the incidence of childhood diseases such as chickenpox, measles, mumps and rubella, varies seasonally $[98,99]$. A standard epidemiological model for the description of these variations, taking into account seasonal variations of the contact rate of children susceptible to 


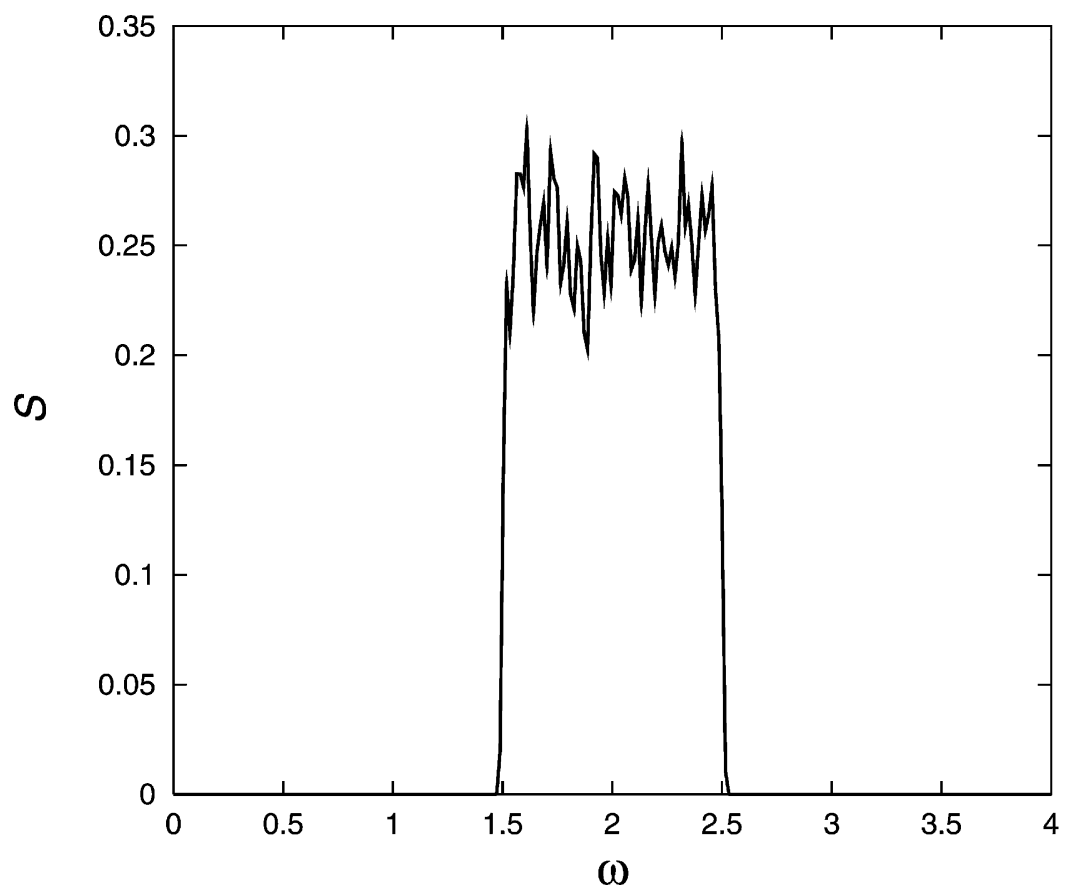

Fig. 17. The spectral density of the noise passed through a bandpass filter.

infection with infective ones, includes four components: (1) susceptibles $(S)$; (2) exposed but not yet infective $(E)$; (3) infective (I); (4) recovered and immune $(R) .{ }^{10}$ Relationships between these components are illustrated schematically in Fig. 19. The relative number of children $S$ susceptible to infection increases with total number of children but decreases because a section of the group remains unexposed and because a section of them falls into the category of the exposed but not yet infective $(E)$. Some of the children exposed remains noninfective, whereas others fall in the category of the infective $(I)$. In its turn, a group of the infective children do not fall sick and another part, having had the disease, recover and falling in the forth category $(R)$. Taking account of the fact that the total number of children is constant, the model equations can be written as

$$
\begin{aligned}
& \dot{S}=m(1-S)-b S I, \quad \dot{E}=b S I-(m+a) E, \quad \dot{I}=a E-(m+g) I, \\
& \dot{R}=g I-m R,
\end{aligned}
$$

where $1 / m$ is the average expectancy time, $1 / a$ is the average latency period, $1 / g$ is the average infection period, $b$ is the contact rate (the average number of susceptibles contacted infection annually). Note that Eqs. (4.72) do not contain the variable $R$; hence these equations can be considered independently of Eq. (4.73).

\footnotetext{
${ }^{10}$ For this reason the model under consideration is often called the SEIR model.
} 

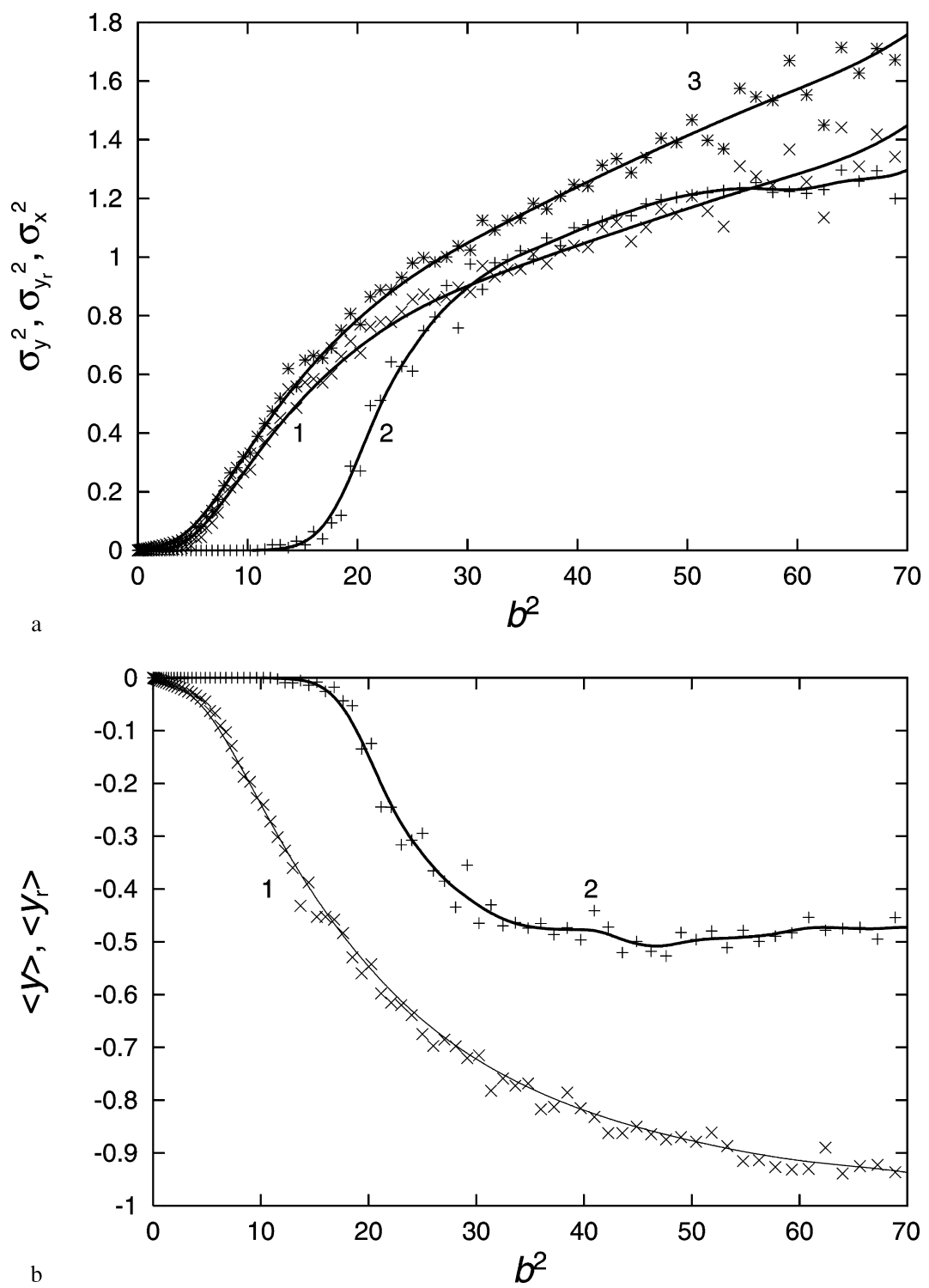

Fig. 18. (a) The numerical dependences of $\sigma_{y}^{2}$ (curve 1), $\sigma_{y_{\mathrm{r}}}^{2}$ (curve 2), and $\sigma_{x}^{2}$ (curve 3) on $b^{2}$ for the noise passed through a bandpass filter. (b) The numerical dependences of $\langle y\rangle$ (curve 1) and $\left\langle y_{\mathrm{r}}\right\rangle$ (curve 2) on $b^{2}$ for the noise passed through a bandpass filter.

Eqs. (4.72) were first considered by Dietz [100], who assumed that the contact rate $b$ varies periodically with the period equal to one year and found analytically periodic oscillations of the model variables. Later these equations were studied in detail by Olsen and Schaffer [101] and Engbert [102]. It was shown that periodic variation of the contact rate can result not only in periodic oscillations of childhood infections but in chaotic behavior as well. 


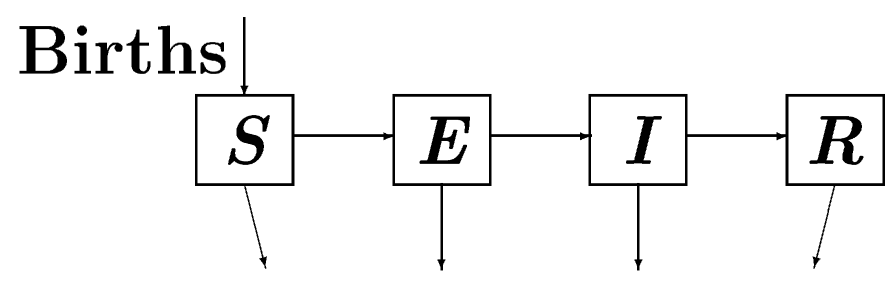

Fig. 19. Diagram illustrating mutual relations between different components in the SEIR model. The relative number $S$ of susceptible children is increased by the birthrate. Of this group, some are not exposed (down-arrow). Of the relative number $E$ that are exposed, some remain noninfective (down-arrow). Of the relative number $I$ that become infective, some do not fall sick but just lose their infectivity again (down-arrow). The relative number that recover $R$ after contracting the disease and falling sick also become noninfective (final down-arrow).

It is easily shown that for a time-independent contact rate $b=$ const $=b_{0}$ Eqs. (4.72) have, depending on the parameters, either one (for $a b_{0} \leq(m+a)(m+g)$ ) or two (for $a b_{0}>$ $(m+a)(m+g))$ singular points: one of them has the coordinates $S=1, E=I=0$ and another (if it exists) has the coordinates

$$
S_{0}=\frac{(m+a)(m+g)}{a b_{0}}, \quad E_{0}=\frac{m}{m+a}-\frac{m(m+g)}{a b_{0}}, \quad I_{0}=\frac{a m}{(m+a)(m+g)}-\frac{m}{b_{0}} .
$$

In the case that there is only one singular point it is stable, whereas in the case that both of the singular points exist the first of them is aperiodically unstable and the second is stable. These cases are said to correspond to extinction of epidemics and endemic equilibrium, respectively.

It is shown in [101] that the values of the model parameters most closely corresponding to the estimates made for childhood diseases in first world countries are $m=0.02 \mathrm{yr}^{-1}, a=35.84 \mathrm{yr}^{-1}$, $g=100 \mathrm{yr}^{-1}, b_{0}=1800 \mathrm{yr}^{-1}$. For these parameters Eqs. (4.72) have two singular points. In our studies we have used these same parameter values.

If the parameter $b$ oscillates then the variables $S, E$ and $I$ oscillate too, and these oscillations occur about the stable singular point with coordinates (4.74). Therefore, it is convenient to substitute into Eqs. (4.72) the new variables $x=S / S_{0}-1, y=E / E_{0}-1$, and $z=I / I_{0}-1$. Putting $b=b_{0}\left(1+b_{1} f(t)\right)$, where $f(t)$ is a function describing the shape of the contact rate oscillation, let us rewrite Eqs. (4.72) in the variables $x, y, z$ :

$$
\begin{aligned}
& \dot{x}+m x=-b_{0} I_{0}\left(\left(1+b_{1} f(t)\right)(x+z+x z)+b_{1} f(t)\right), \\
& \dot{y}+(m+a) y=(m+a)\left(\left(1+b_{1} f(t)\right)(x+z+x z)+b_{1} f(t)\right), \\
& \dot{z}+(m+g) z=(m+g) y .
\end{aligned}
$$

In Eqs. (4.75) the term $b_{1} f(t)$ can be considered as an external action upon the system. We see from (4.75) that this action is not only multiplicative, i.e., parametric, but additive, i.e., forcing, as well. It should be noted that, owing to the quadratic nonlinearity, the forcing action can cause a strong response of the system even in the absence of resonance.

For $b_{1}=0$ and small initial deviations from the steady state $x=0, y=0, z=0$ the system executes damped oscillations which are close to harmonic in shape (Fig. 20(a)). The frequency of these oscillations $\omega_{0} \approx \pi$. As initial deviations increase the natural oscillations of the system 

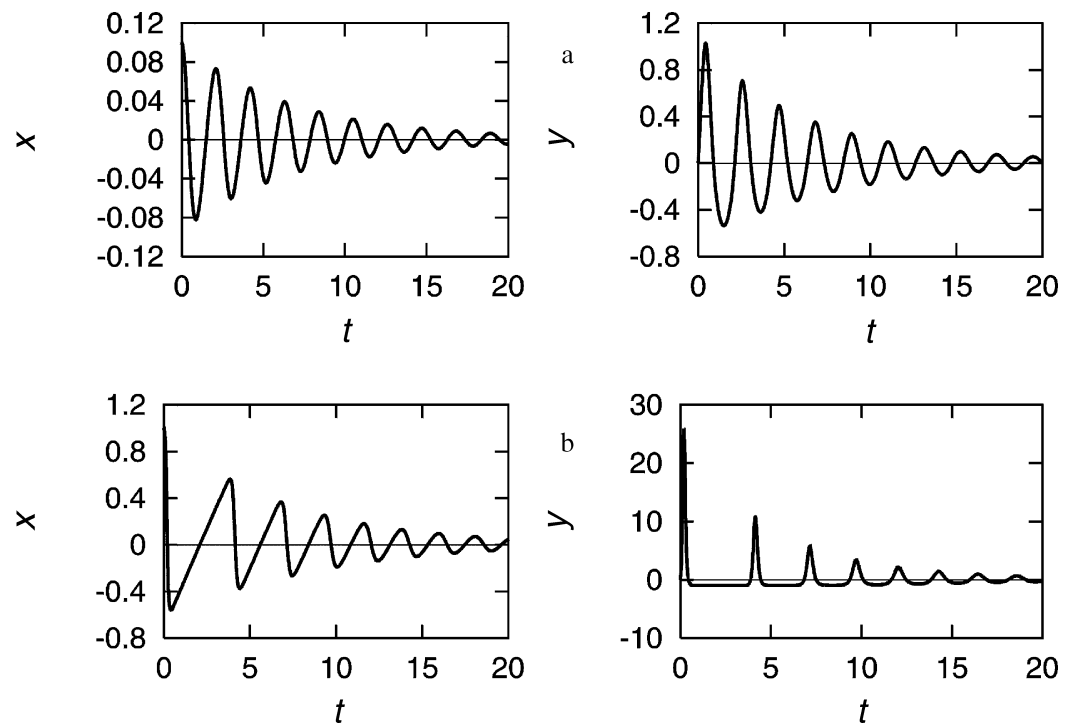

Fig. 20. Natural oscillations of the SEIR model variables $x$ and $y$ for $y(0)=0, z(0)=0$ and: (a) $x(0)=0.1 ;(b) x(0)=1$. The values of the parameters are determined by (4.75). The time-evolution of the variable $z$ is similar to that of $y$.

deviate further and further from harmonicity, as exemplified by Fig. 20(b). The frequency of natural oscillations decreases with their increasing amplitude.

As mentioned above, in $[100,101]$ it was assumed that, owing to seasonal variations of environmental conditions, the contact rate $b$ depends periodically on time with a period equal to one year, viz., $f(t)=\cos \omega t$, where $\omega=2 \pi$. We emphasize that the frequency of the contact rate variation is about twice the natural frequency of small free oscillations of the model variables $\omega_{0}$.

It was shown that the periodic variation of the parameter $b$ causes the appearance either periodic or chaotic oscillations of the variables $S, E$, and $I$. For very small $b_{1}$ the oscillations excited are close to harmonic at the frequency of the action $\omega$. For a certain value of $b_{1}$ a period-doubling bifurcation occurs that is associated with the parametric mechanism of the oscillation excitation. As $b_{1}$ increases the main frequency of the oscillations remains equal to $\omega / 2$ and the shape of the oscillations of the variable $x$ approaches a saw-tooth. On further increasing $b_{1}$ another perioddoubling bifurcation takes place, and then a drastic transition to chaos, accompanied by a dramatic increase in the oscillation variance, occurs. We note that chaotic oscillations for $b_{1}=0.28$ were first found numerically by Olsen and Schaffer [101]. For this value of $b_{1}$ the time dependences of $x$ and $y$, and the projection of the phase trajectory on the $x, y$-plane found by numerical simulation of Eqs. (4.75) are shown in Fig. 21(a).

From a physical standpoint, an assumption of random variation of the contact rate seems more justified than a periodic variation. It is evident that $b(t)$ has to be a sufficiently wide-band random process for which the spectral density peaks at the frequency corresponding to one reciprocal year. Starting from this assumption we have simulated numerically Eqs. (4.75) with $f(t)=\chi(t)$, where $\chi(t)$ is a random process which is a solution of the equation

$$
\ddot{\chi}+2 \pi \dot{\chi}+6 \pi^{2} \chi=k \xi(t),
$$



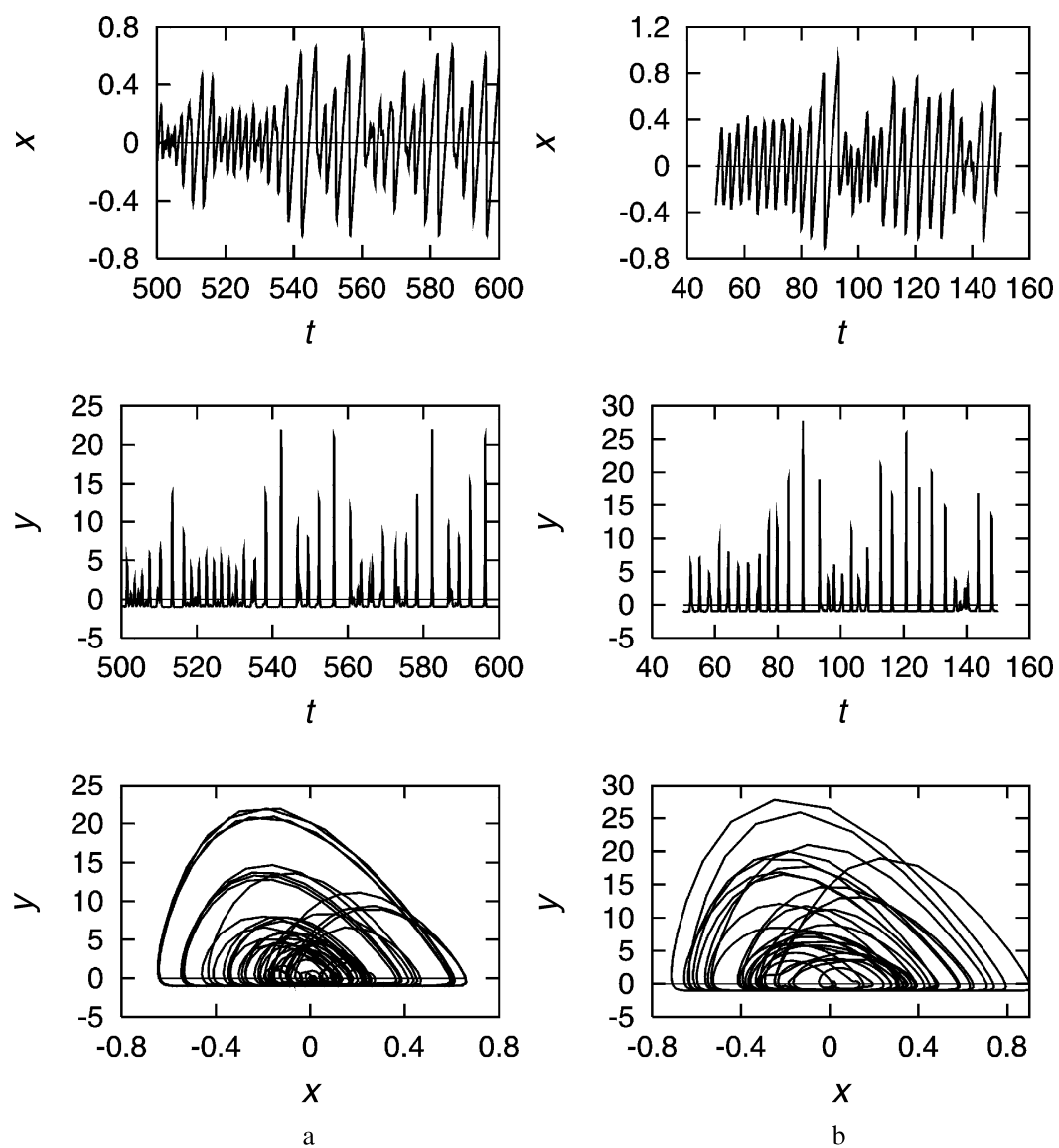

Fig. 21. The time dependences of the variables $x$ and $y$, and the projection of the phase trajectory on the $x, y$-plane for: (a) $f(t)=\cos 2 \pi t, b_{1}=0.28$; and (b) $f(t)=\chi(t), b_{1}=0.235$.

$\xi(t)$ is white noise, and $k$ is a factor which we choose such that the variance of $\chi(t)$ is equal to $\frac{1}{2}$. The resultant plots of $\chi(t)$ and its spectral density are shown in Fig. 22.

The results of numerical simulation of Eqs. (4.75) with $f(t)=\chi(t)$ are shown in Fig. 21(b) for the same values of the parameters as in Fig. 21(a) and $b_{1}=0.235$ chosen so that the variance of $x(t)$ would be approximately the same as for $f(t)=\cos 2 \pi t, b_{1}=0.28$. It is seen from this figure that the noise-induced oscillations differ very slightly in their form from those for the case of a harmonic variation of the contact rate. This is associated with the fact that the variation of the contact rate only induces the phase transition, whereas the shape of the induced oscillations is mainly determined by intrinsic properties of the system, manifesting themselves in the shape of free oscillations as well.

The evolution of the oscillation power spectra in the case of a periodic variation of the contact rate is illustrated in Fig. 23. We see that, for $b_{1}=0.03$, the spectral density does peak at the frequency $\omega$ whereas, for $b_{1}=0.1$, it peaks at the frequency $\omega / 2$. For $b_{1}=0.26$ the power spectrum 

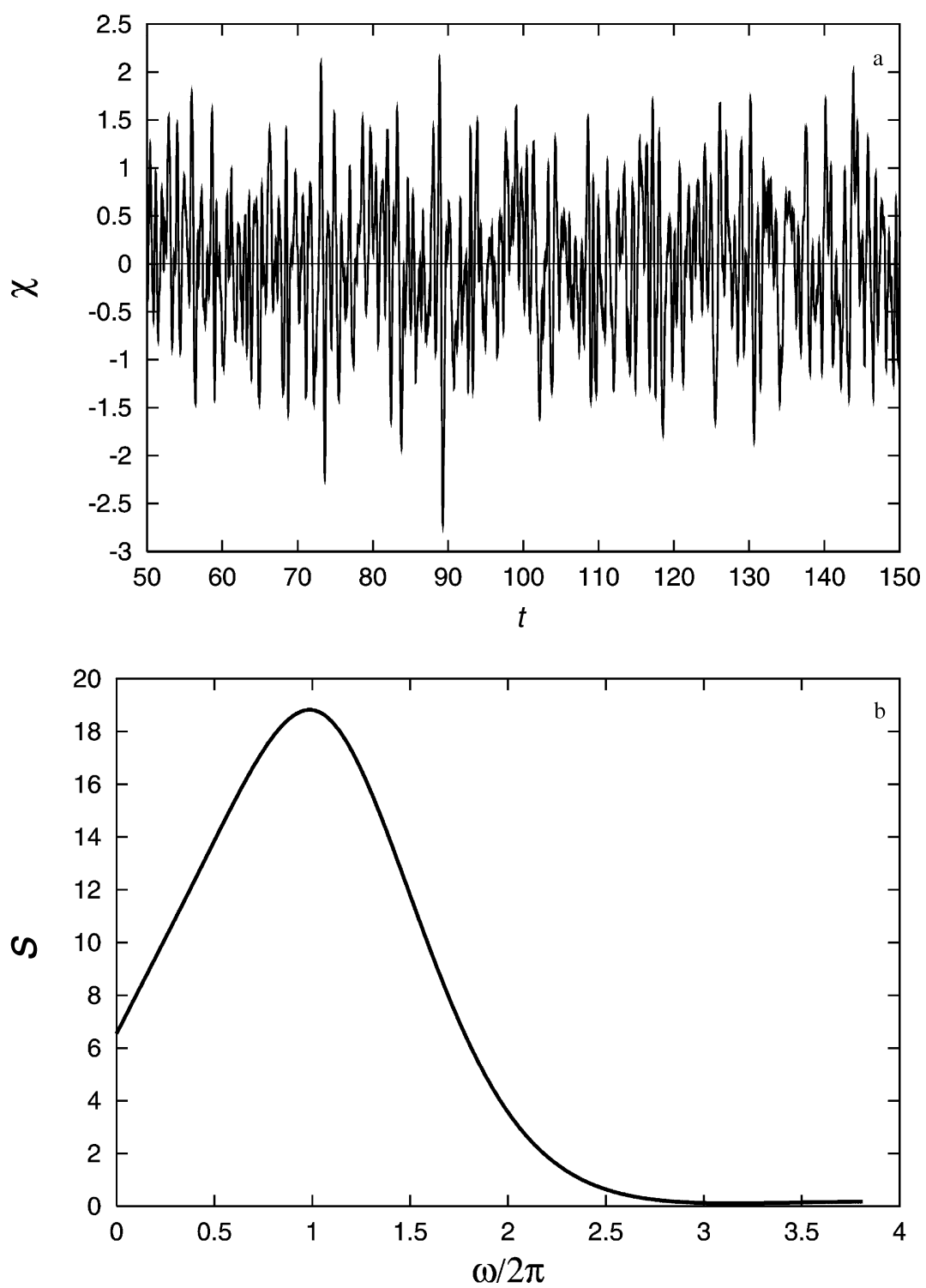

Fig. 22. Plots (a) of $\chi(t)$ and (b) of its spectral density $S(\omega)$.

contains the forth subharmonic, and for $b_{1}=0.28$, when the oscillations are chaotic, the spectrum becomes continuous with a maximum at the frequency $\omega / 4$.

The excitation of oscillations at the frequency $\omega / 2=\pi$ can be considered as a second-order phase transition induced by the periodic variation of the contact rate. This is supported by the dependence of the variance of the variable $x$, which we denote by $\sigma^{2}$, on the parameter $b_{1}$ (Fig. 24(a)). We see that for $b_{1} \approx 0.066$ the rate of change of the variance increases markedly. Close to this point the dependence of $\sigma^{2}$ on $b_{1}$ can be approximated by the formula 

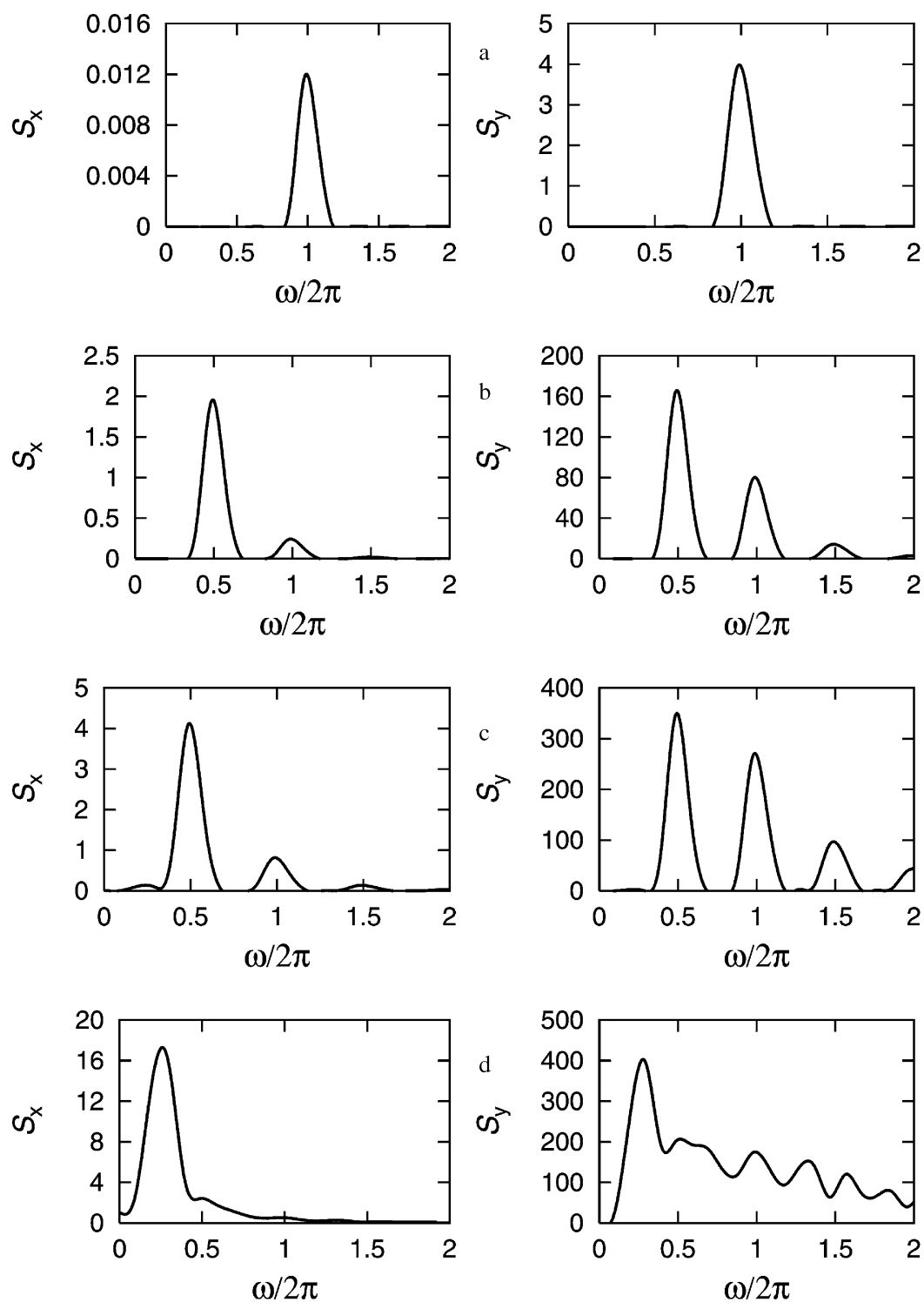

Fig. 23. The evolution of the oscillation power spectra of $x(t)$ and $y(t)$ in the case of a harmonic variation of the contact rate for: (a) $b_{1}=0.03$, (b) $b_{1}=0.1$, (c) $b_{1}=0.26$, and (d) $b_{1}=0.28$.

$\sigma^{2}=0.038\left(b_{1}-0.066\right)^{1 / 2}$. So, the critical index is equal to $1 / 2$. Such a dependence is typical of second-order phase transitions: one can consider $\sigma^{2}$ as an analog of the order parameter and $b_{1}$ as an analog of the temperature. For $b_{1} \approx 0.27$ another transition, revealing itself in going from periodic oscillations to chaotic ones and in a jump-like increase of the variance, occurs (see Fig. 24(b)). This transition can be considered as an induced first-order phase transition.

The evolution of the oscillation power spectra in the case of random variation of the contact rate is given in Fig. 25. In contrast to what happens when the variation of the contact rate is periodic, 

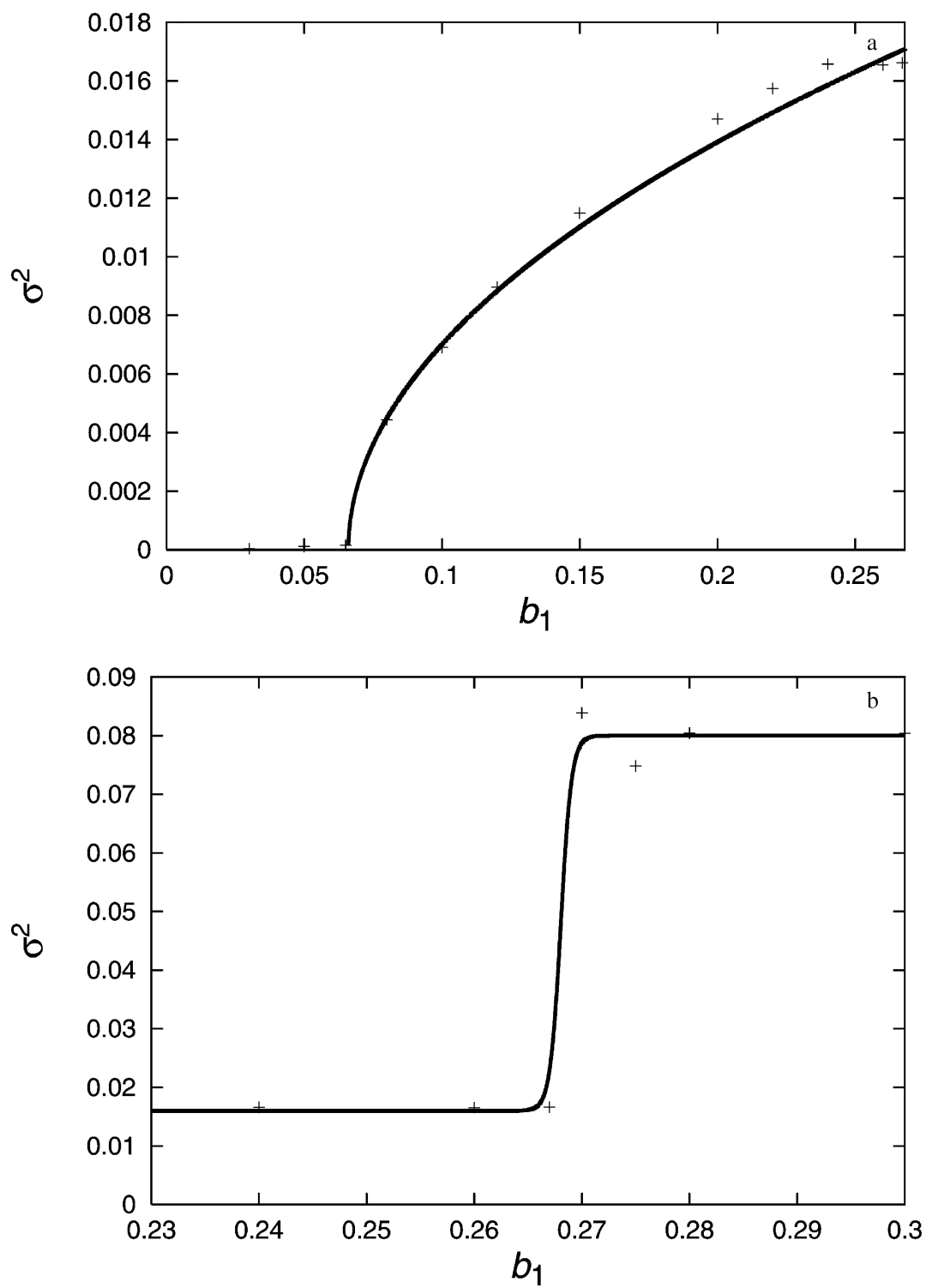

Fig. 24. The dependence of the variance of $x(t)\left(\sigma^{2}\right)$ on the parameter $b_{1}$ : (a) in the range $0 \leq b_{1} \leq 0.268$; and (b) in the range $0.23 \leq b_{1} \leq 0.3$. The dependence $\sigma^{2}=0.038\left(b_{1}-0.066\right)^{1 / 2}$ is shown in (a) as a solid line.

in this case the power spectra are always continuous, even if have a number of maxima. As $b_{1}$ increases the main maximum of the spectrum shifts to lower frequencies.

As with the periodic variation of the contact rate, a random variation also induces a phase transition [103]. The dependence of the variance of $x(t)$ on the parameter $b_{1}$ is shown in Fig. 26. In a certain range of $b_{1}$ this dependence can be approximated by a straight line. The value of $b_{1}$ for which this straight line intersects the abscissa is equal to 0.066. Such a dependence is typical of noisy second-order phase transitions. 

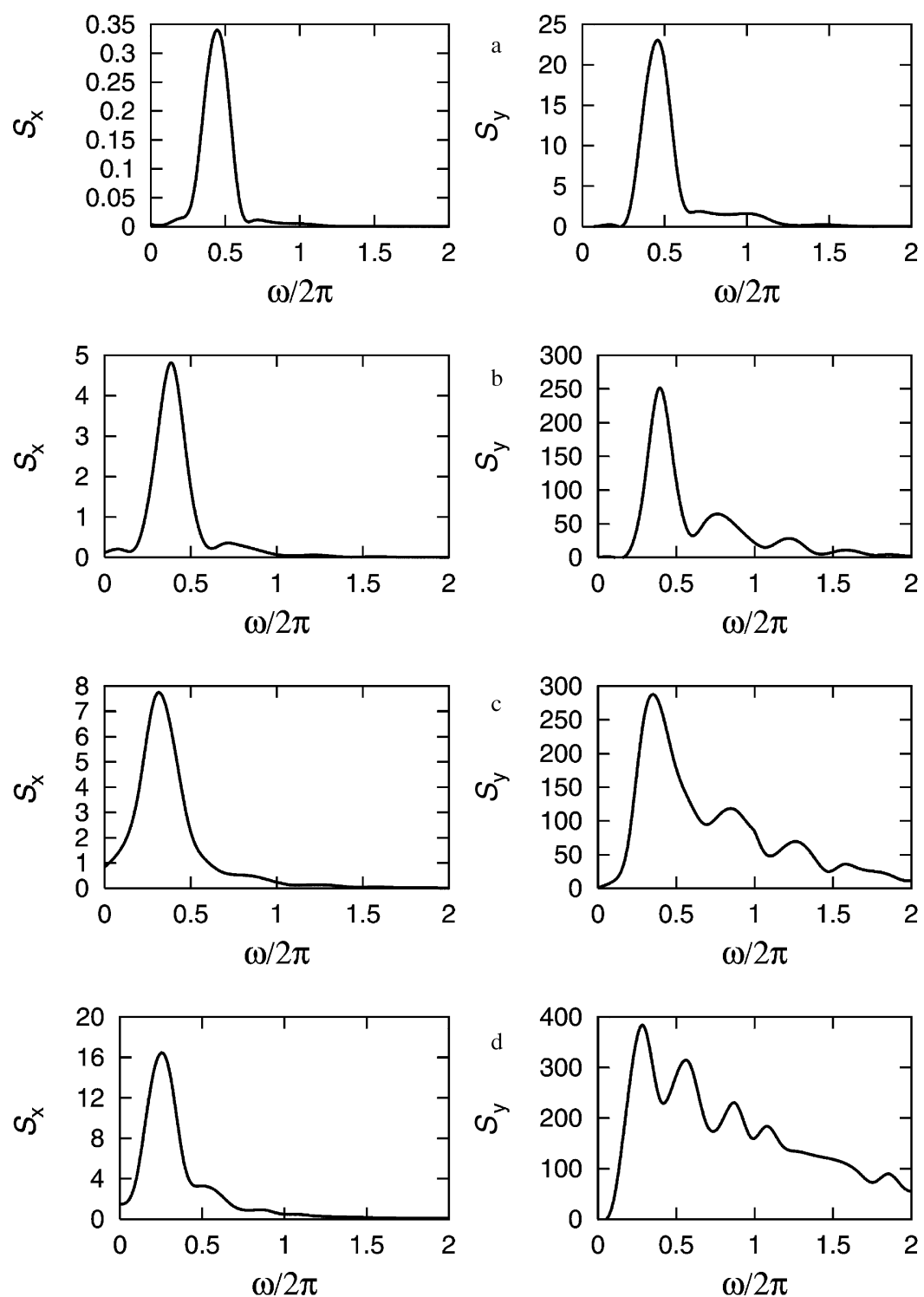

Fig. 25. The evolution of the power spectra of $x(t)$ and $y(t)$ for the case of random variation in the contact rate, with: (a) $b_{1}=0.03$; (b) $b_{1}=0.1$; (c) $b_{1}=0.2$; and (d) $b_{1}=0.235$.

To clarify the physical mechanisms responsible for the phase transitions, let us change somewhat Eqs. (4.75) so that the amplitudes of parametrical and forcing actions can be varied independently. Namely, let us rewrite Eqs. (4.75) in the form

$$
\begin{aligned}
& \dot{x}+m x=-b_{0} I_{0}\left(\left(1+b_{1} f(t)\right)(x+z+x z)+b_{2} f(t)\right), \\
& \dot{y}+(m+a) y=(m+a)\left(\left(1+b_{1} f(t)\right)(x+z+x z)+b_{2} f(t)\right), \\
& \dot{z}+(m+g) z=(m+g) y .
\end{aligned}
$$




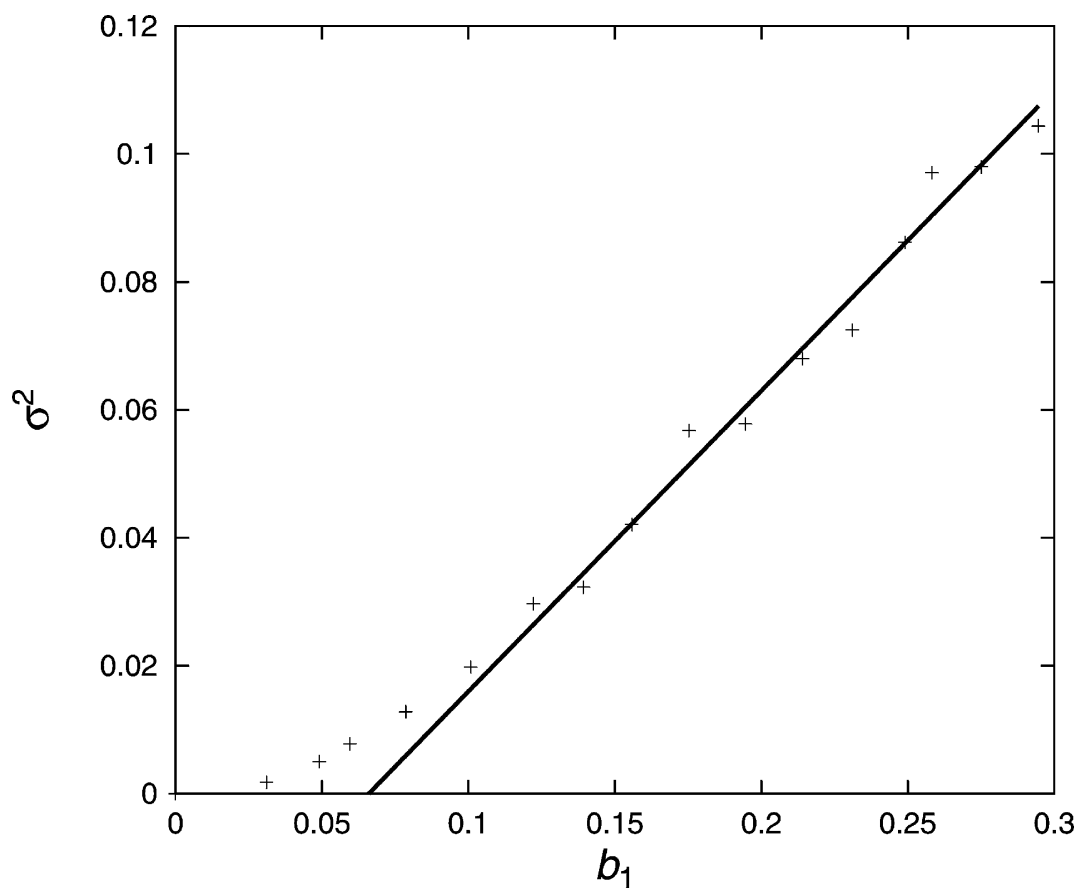

Fig. 26. The dependence of $\sigma^{2}$ on the parameter $b_{1}$ in the case of a random variation of the contact rate. The solid line represents $\sigma^{2}=0.47\left(b_{1}-0.066\right)$.

First we consider the case when the additive action is absent, i.e., $b_{2}=0, b_{1} \neq 0$. In this case the dependence of $\sigma^{2}$ on $b_{1}$ for periodic variation of the contact rate is shown in Fig. 27(a). It is evident from this figure that oscillations are excited only when a critical value of the parameter $b_{1}$ is exceeded. This is the characteristic property of parametrically excited oscillations (see, for example, [1]). With increasing difference between $b_{1}$ and its critical value $b_{1}^{(\text {cr) }} \approx 0.032$ the variance increases nearly linearly. So, the critical index for this case is equal 1 . For $b_{1}>0.075$ the solution becomes unstable and goes to infinity.

If there is only additive action, i.e., $b_{1}=0, b_{2} \neq 0$, oscillations are excited even for values of $b_{2}$ as small as is wished. However, for $b_{2}<b_{2}^{\text {(cr) }}$, where $b_{2}^{(\text {cr) }} \approx 0.0885$, the amplitude of these oscillations is very small and their frequency is equal to $\omega$. For $b_{2} \approx b_{2}^{\text {(cr) }}$ the rate of change of the variance increases rapidly and for $b_{2}>b_{2}^{(\text {cr) }}$ the dependence of $\sigma^{2}$ on $b_{2}$ can be approximated by the straight line $\sigma^{2}=0.14\left(b_{2}-b_{2}^{(\mathrm{cr})}\right)$ (see Fig. 27(b)). The drastic increase in the rate of change of the variance is associated with the onset of subharmonic resonance. Indeed, the main frequency of the oscillations excited, for $b_{2}>b_{2}^{\text {(cr) }}$, becomes equal to $\omega / 2$. Considering this process as a second-order phase transition, we can conclude that, as with purely parametric action, in the case of purely additive action the critical index is also equal to 1 . For $b_{2}>0.15$ the solution, as in the case of parametrical excitation, becomes unstable and goes to infinity. The computation of Eqs. (4.77) with $b_{1} \neq 0$ and $b_{2} \neq 0$ shows that parametric and additive actions affect the behavior in opposite directions, resulting in the stabilization of the solution for moderately large amplitudes. The combined effect of these actions is described above. 

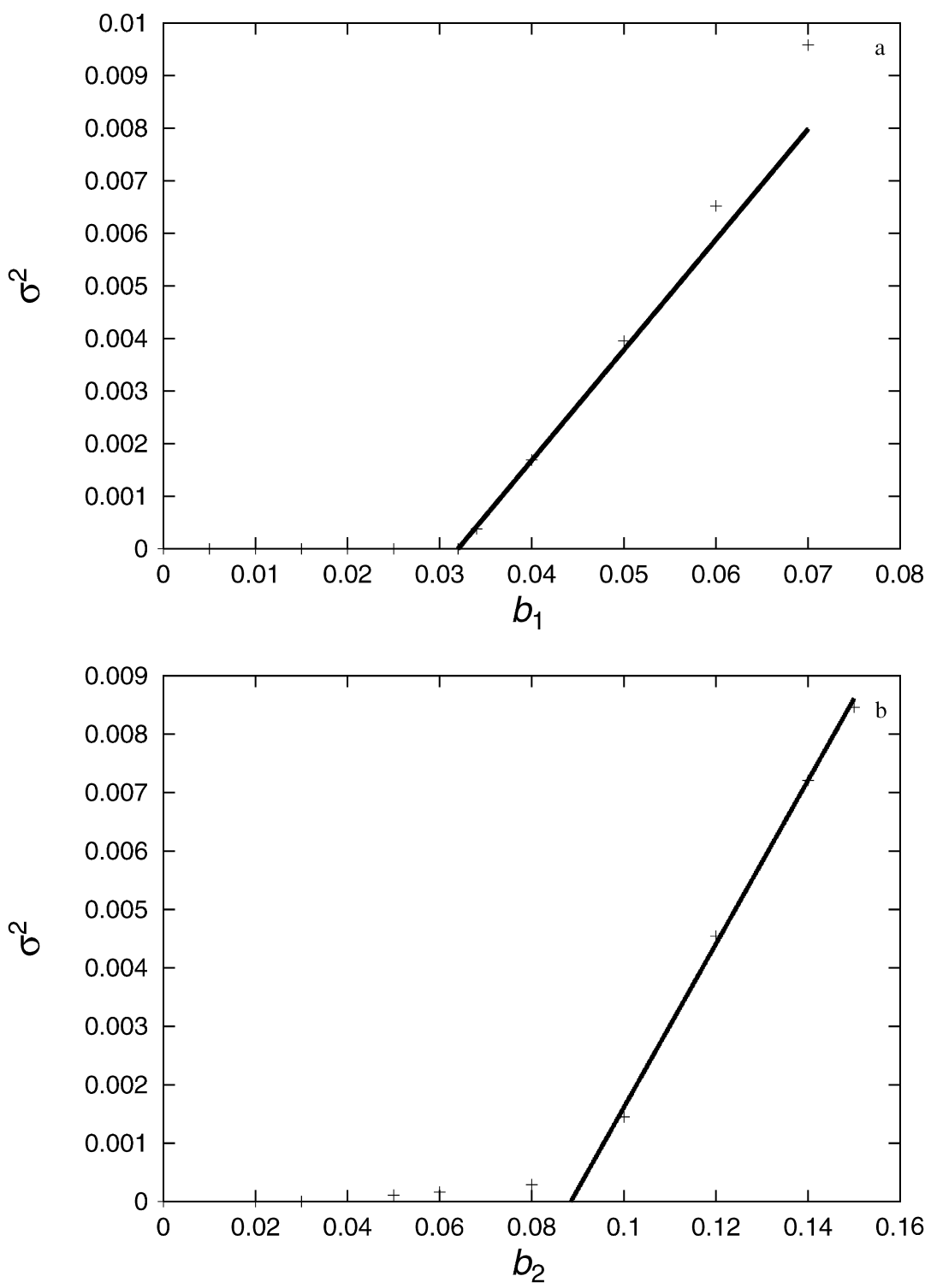

Fig. 27. Plot of $\sigma^{2}$ versus the action amplitude for (a) parametrically excited oscillations; and (b) additively forced oscillations. The dependencies $\sigma^{2}=0.21\left(b_{1}-0.032\right)$ and $\sigma^{2}=0.14\left(b_{2}-0.0885\right)$ are shown by the solid lines in parts (a) and (b), respectively.

In the case of random variation of the contact rate we have also attempted to simulate Eqs. (4.77) with $f(t)=\chi(t)$ and put $b_{2}=0, b_{1} \neq 0$ and vice versa. It is found that, in the case of only multiplicative action, the phase transition occurs via on-off intermittency as for a pendulum with randomly vibrated suspension axis $[38,39]$. The critical value of the parameter $b_{1}$ is approximately equal to 0.095 , i.e., significantly larger than in the case of harmonic variation of the contact rate. An example of oscillations of the variables $x$ and $y$ for $b_{1}=0.099$, illustrating on-off intermittency, is 
given in Fig. 28. Unfortunately, the determination of the dependence of the oscillation variance on $b_{1}$ appears to be impossible because, even for $b_{1}=0.1$, the solution goes to infinity. In the case, when $b_{1}=0, b_{2} \neq 0$, the system behaves in a similar manner: the solution goes to infinity for $b_{2} \geq 0.08$. Nevertheless, we can conclude that the multiplicative component of the random action induces a phase transition much as in a pendulum with randomly vibrated suspension axis, whereas the additive component induces a phase transition much as in a nonlinear oscillator with
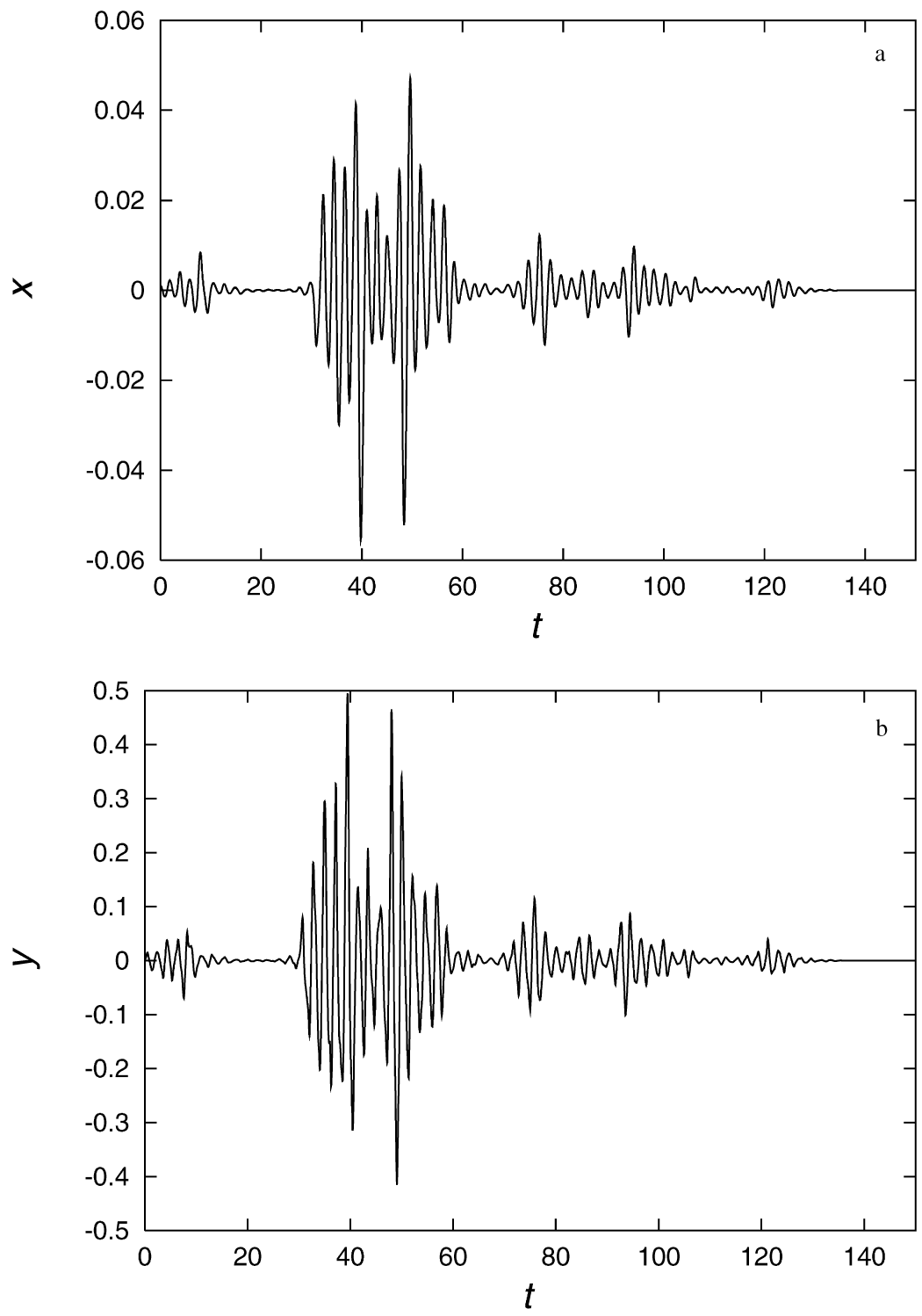

Fig. 28. An example of oscillations of the variables $x$ and $y$ for $b_{1}=0.099$ for the case of purely multiplicative random action. 
quadratic nonlinearity. The joint action of both components results in the dependence of the variance of $x(t)$ on the parameter $b_{1}$ which is shown in Fig. 26.

\subsubsection{Noise-induced oscillations in a Bonhoeffer-van der Pol oscillator}

The equations that came to be known as those for a Bonhoeffer-van der Pol oscillator were suggested by Bonhoeffer for simulating neural pulses [104-107]. They are a generalization of the van der Pol equations for relaxation oscillations [108], and describe oscillations of the voltage across a neural membrane $x$ allowing for the refractoriness characterized by the variable $y$. Subsequently, similar equations, but incorporating spatial diffusion, came to be known as the Fitz Hugh-Nagumo equations [109-111]. The Bonhoeffer-van der Pol equations can be written as [112]

$$
\dot{x}=x-\frac{x^{3}}{3}-y+I(t), \quad \dot{y}=c(x+a-b y),
$$

where $a, b$, and $c$ are the membrane radius, the specific resistivity of the fluid inside the membrane, and the temperature factor, respectively; $I(t)=I_{0}+F(t)$ is the current across the membrane with $I_{0}$ being the direct component of this current. For $F(t)=0$ and the parameters corresponding to real membranes (following, for example, [113] we set $a=0.7, b=0.8$, and $c=0.1$ ). Eq. (4.78) have one singular point $x=x_{0}, y=y_{0}=(x+a) / b$, where $x_{0}$ is a real root of the equation

$$
\frac{x^{3}}{3}+\left(\frac{1}{b}-1\right) x+\frac{a}{b}-I_{0}=0 .
$$

In the ranges $I_{0}<0.341$ and $I_{0}>1.397$ this point is a stable focus; whereas for $0.341<I_{0}<1.397$ it is an unstable focus.

Setting $\xi=x-x_{0}, \eta=y-y_{0}$ we obtain for $\xi$ and $\eta$ the following equations:

$$
\dot{\xi}=-\left(\frac{\xi^{3}}{3}+x_{0} \xi^{2}+\left(x_{0}^{2}-1\right) \xi+\eta\right)+F(t), \quad \dot{\eta}=c(\xi-b \eta) .
$$

The case when the system under consideration is not self-oscillatory is of prime interest for the purpose of this paper. Therefore we restrict our consideration to the value of $I_{0}$ equal to 0.2 . Eqs. (4.80) are notable for that they have two exceptional phase trajectories. One of these trajectories has a positive Lyapunov exponent, i.e., it is unstable, whereas the other is stable. The first trajectory has a part which repels all neighboring phase trajectories and, conversely, the second has two parts which attract all neighboring phase trajectories. It is evident that these exceptional trajectories are not a repeller and attractor in the strict sense, because the system described by Eqs. (4.80) for $I_{0}=0.2$ has no repellers and only a single attractor: the stable singular point. However, due to their similarity to an ordinary repeller and attractor they have been called [114] a transient repeller and transient attractor, respectively. We will follow this convention. ${ }^{11}$

To find the transient repeller numerically, we can reverse the direction of time. As a result, we obtain the phase plane picture shown in Fig. 29(a). The part which repels all neighboring phase

\footnotetext{
${ }^{11}$ We note that there is some variability in the names of such trajectories. For example, in [115] they are called local separatrices and local attractors, whereas in [116] the attracting trajectory is called phantom attractor and so on.
} 

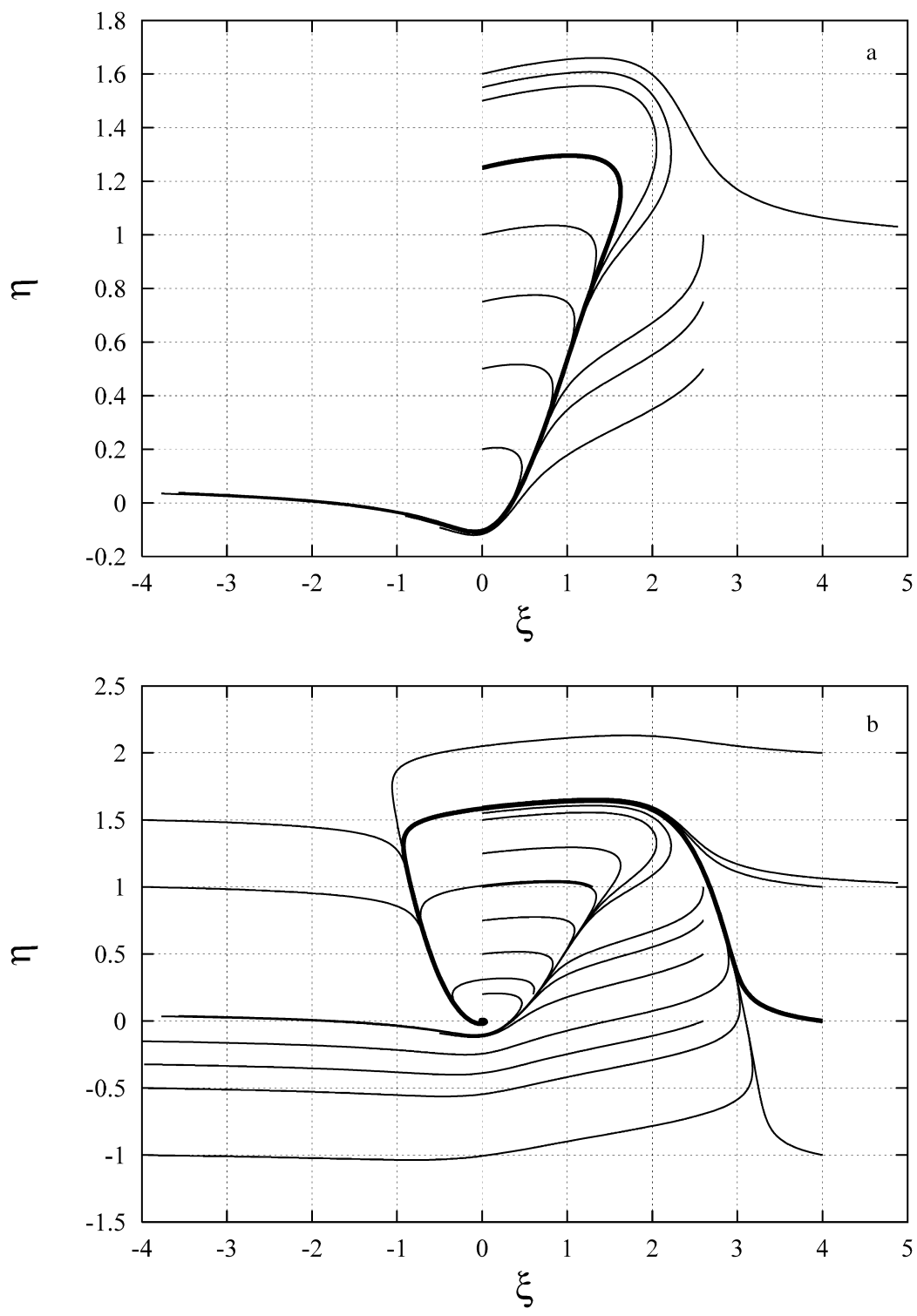

Fig. 29. (a) The transient repeller and neighboring phase trajectories, and (b) the phase portrait involving the transient repeller and transient attractors. In each case, $a=0.7, b=0.8, c=0.1$ and $I_{0}=0.2$.

trajectories is shown as a thick solid line. A full phase portrait involving the trajectories with attracting and repelling parts is given in Fig. 29(b). The transient attractor is shown as a thick solid line. We see that the transient repeller separates the regions of deviations from the equilibrium state corresponding to radically different transient processes.

If the current across the membrane contains an alternating component, for example, $F(t)=A \cos \omega t$ then, from a certain critical value of $A$ onward oscillations are excited, associated 
with the motion of the representative point along the transient attractor [117,118]. It follows from the results of numerical simulation of Eq. (4.80) that the variance of the variables $\xi$ and $\eta$ increases almost discontinuously in this process. An example of the dependence of the variance of the variable $\xi$ on $A$ for $\omega=0.3$ is given in Fig. 30(a). The excitation of such oscillations, accompanied by a drastic increase in the oscillation variance, can be considered as a first-order phase transition. It is interesting that the jump exhibits fine structure: there are local ups and downs in the variance growth (see Fig. 30(b) and (c)). This fine structure is associated with drastic changes in the oscillations' shape under small changes of $A$ (see Fig. 31).

The critical value of $A$ depends on the frequency $\omega$ : it is minimal for a certain value of the frequency which in turn depends on $I_{0}$. The reason of this dependence lies in the resonant response of the nonlinear oscillator to a harmonic external force. As an example, the dependence of the critical value of the amplitude $A$ on the frequency $\omega$ is shown in Fig. 32 for $I_{0}=0.2$. We see that the critical value of the amplitude is minimal for $\omega=0.27$, which is close to the frequency $\omega_{0} \approx 0.3146$ of small free oscillations about the equilibrium state.

If the alternating component of the current across the membrane $F(t)$ is a random process, for example white noise, then the transition to a new state occurs too, but is of radically different character. The appearance of a "limit cycle" induced by white noise was considered in $[115,119,97]$. However, these works are mainly devoted to the calculation of the probability distributions in the vicinity of the induced "limit cycle". We consider this phenomenon in terms of how it exhibits the intrinsic properties of the system and from the standpoint of a noise-induced
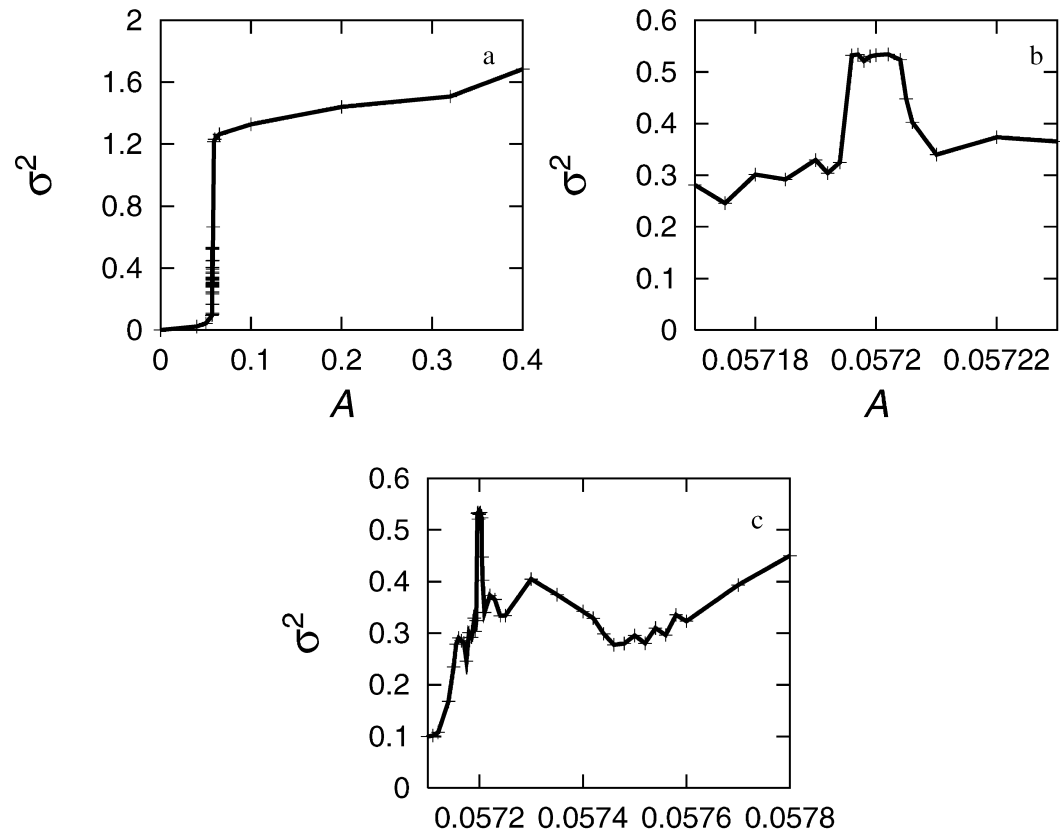

A

Fig. 30. The dependence of the variance of the variable $\xi\left(\sigma^{2}\right)$ on the amplitude $A$ of a periodic component of the current across the membrane for $\omega=0.3$ in the ranges: (a) $0 \leq A \leq 0.4$; (b) $0.05717 \leq A \leq 0.05723$; and (c) $0.0571 \leq A \leq 0.0578$. 

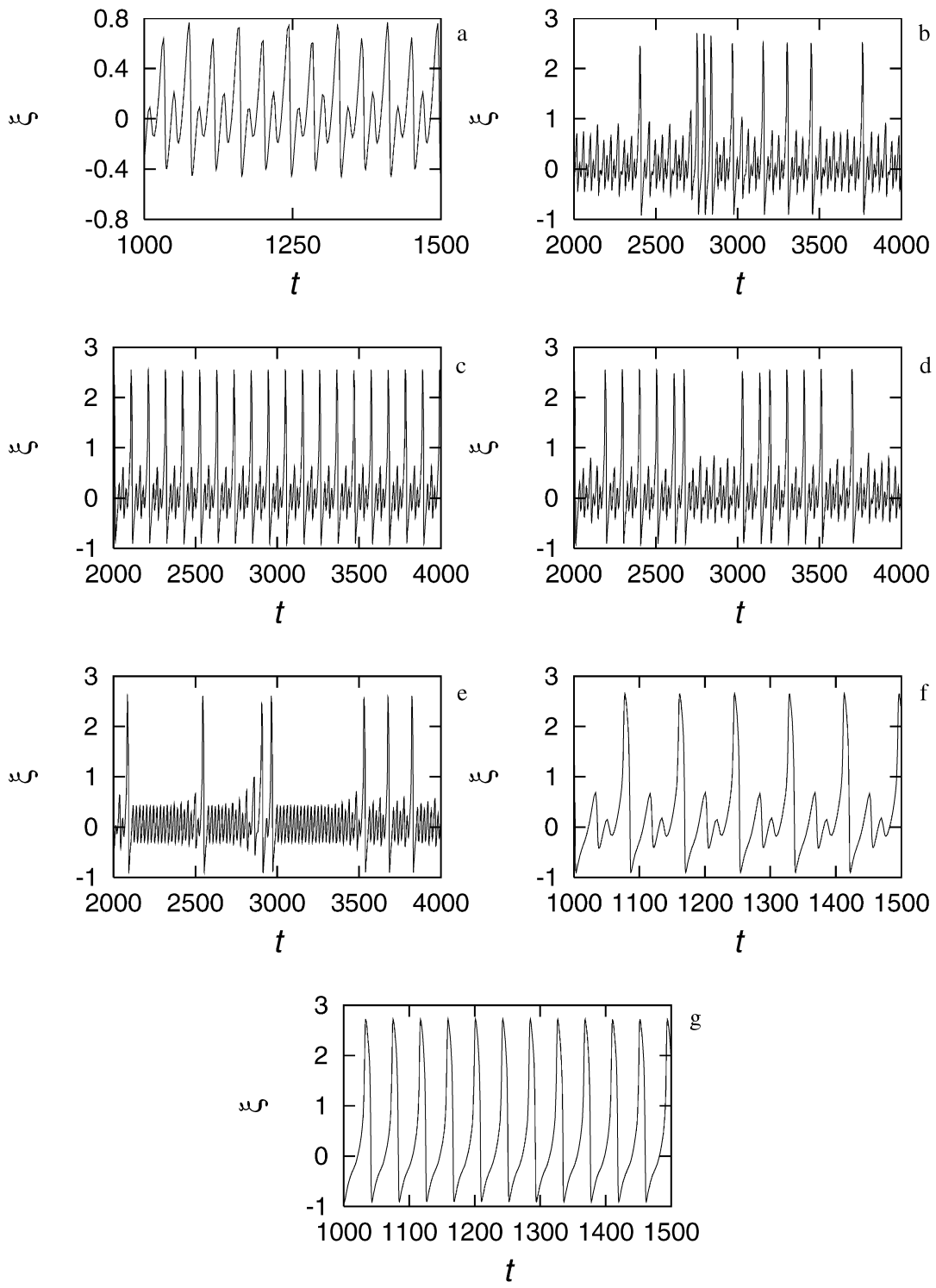

Fig. 31. Plots of $\xi(t)$ for $\omega=0.3$ and: (a) $A=0.057$; (b) $A=0.05717$; (c) $A=0.0572$; (d) $A=0.05723$; (e) $A=0.05746$; (f) $A=0.058$; and (g) $A=0.059$.

transition to a new state. The latter is associated with the crossing of a boundary on the phase plane (the transient repeller is such a boundary) by the representative point under action of noise. In principle, such an intersection is possible for an arbitrarily small noise intensity $\kappa$. Therefore the transition occurs smoothly from $\kappa=0$ onward and hence, in a strict sense this process is not a phase transition. Nonetheless, it is closely similar to a second-order noise-induced phase transition. For example, the dependence of the variance of the variable $\xi$ on the noise intensity $\kappa$, 


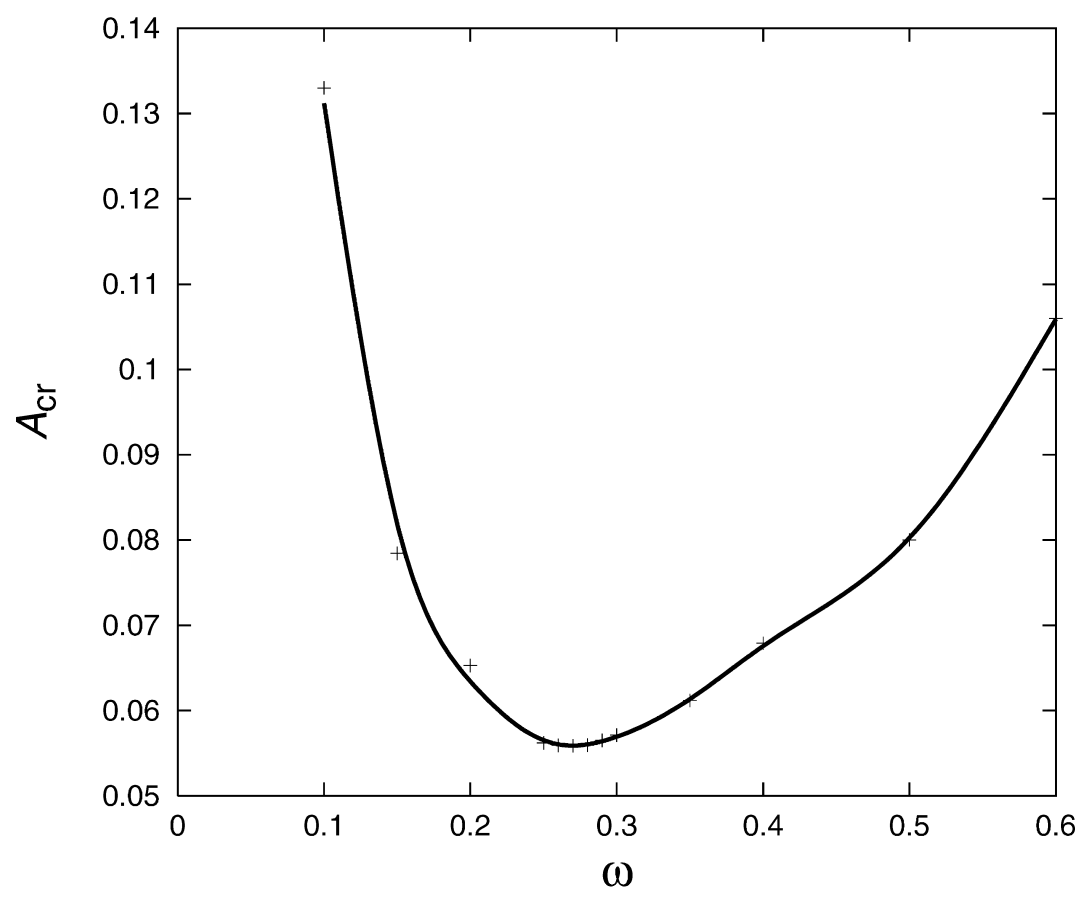

Fig. 32. The dependence of the critical amplitude value $A_{\text {cr }}$ on the frequency $\omega$ for $I_{0}=0.2$.

found by numerical simulation of Eqs. (4.80) and shown in Fig. 33, can be approximated on a certain interval by $\sigma_{\xi}^{2} \approx 5\left(\kappa-\kappa_{\mathrm{cr}}\right)^{1 / 2}$, where $\kappa_{\mathrm{cr}} \approx 0.0065$. This formula is similar to that describing the dependence of an order parameter on temperature for conventional second-order phase transitions, with a critical index equal to $1 / 2$. Furthermore, it can be seen (see, e.g., Fig. 34) that this transition occurs via a peculiar kind of on-off intermittency. Just as for ordinary on-off intermittency $[56,59,38,39]$, close to the onset of the transition the representative point on the phase plane is moving within a certain $\varepsilon$-vicinity of the equilibrium state over prolonged periods (so called "laminar phases"), and only occasionally escapes from this vicinity. Unlike ordinary on-off intermittency, however, these escapes have not random but strictly specified shape of pulses, and their durations are unchanged as the noise intensity increases. That is why these escapes should not be called "turbulent phases". Away from onset, the duration of the laminar phases decreases and the variance of the system variables increases. Because the duration of the pulses is unchanged, we can use the mean interpulse time (the mean period) in place of the mean duration of the laminar phases. The dependence of the mean period $T$ on the noise intensity $\kappa$ is shown in Fig. 35. It can be approximated by the formula

$$
T \approx 38 \exp (0.02 / \kappa),
$$

which is typical for the mean time for reaching a boundary (see Section 1). We see that the mean period decreases exponentially as the noise intensity increases. This result coincides qualitatively with the initial part of the corresponding dependence obtained in $[115,119]$. However, we have not found an increase of the mean period with increasing the noise intensity as reported in $[115,119]$. 


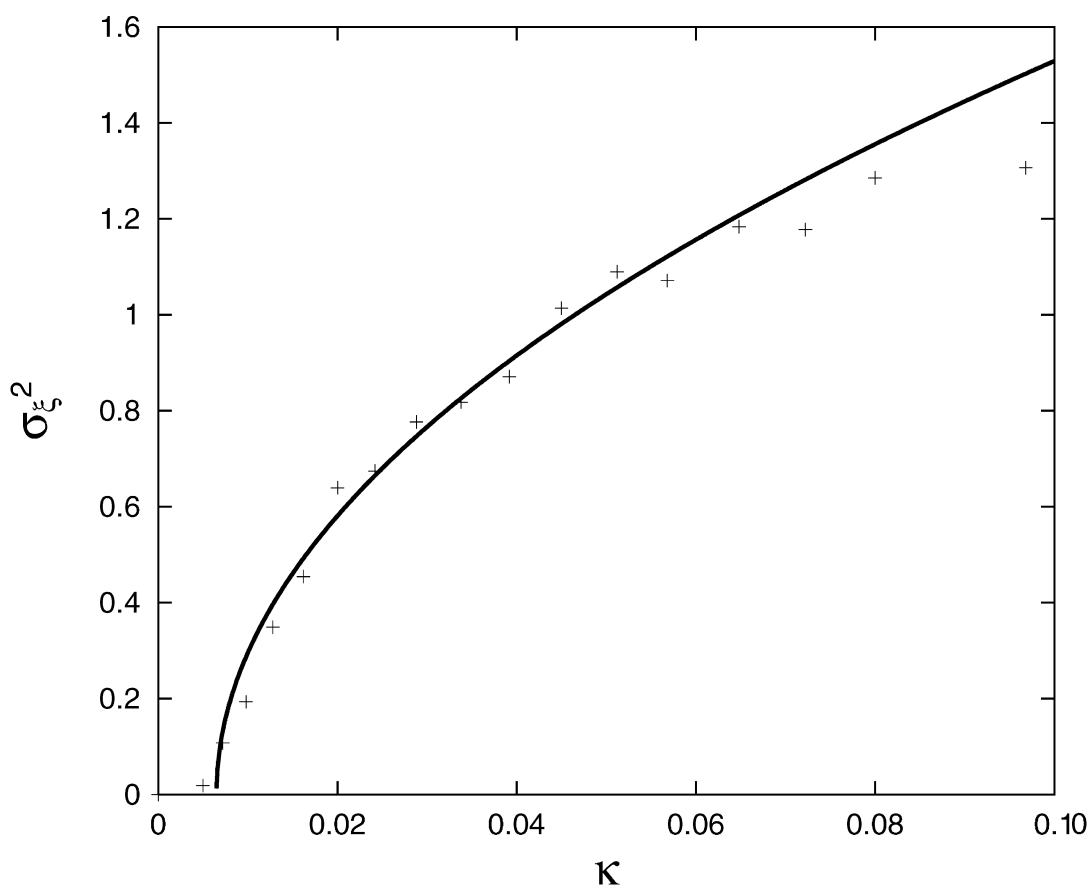

Fig. 33. The dependence of the variance of the variable $\xi$ on the noise intensity $\kappa$. The solid curve plots $\sigma_{\xi}^{2}=5 \sqrt{\kappa-0.0065}$.

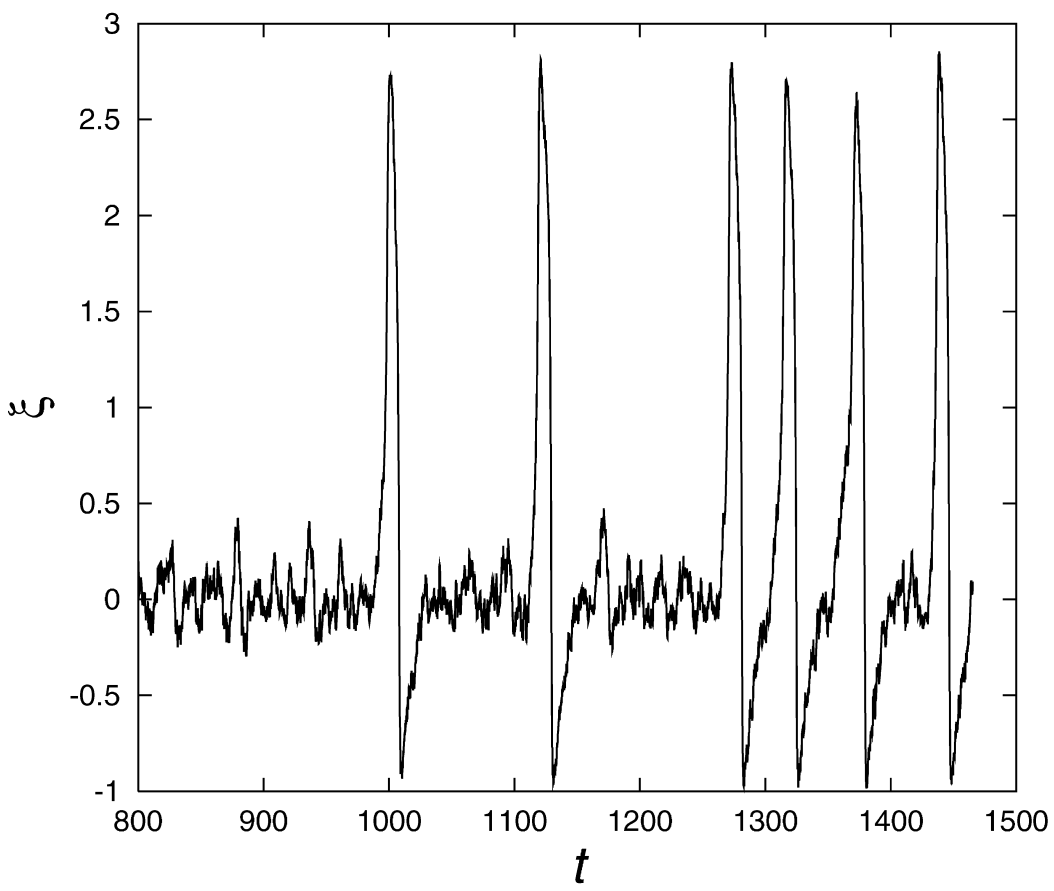

Fig. 34. An example of oscillations of the variable $\xi(t)$ for $\kappa=0.0098$. 


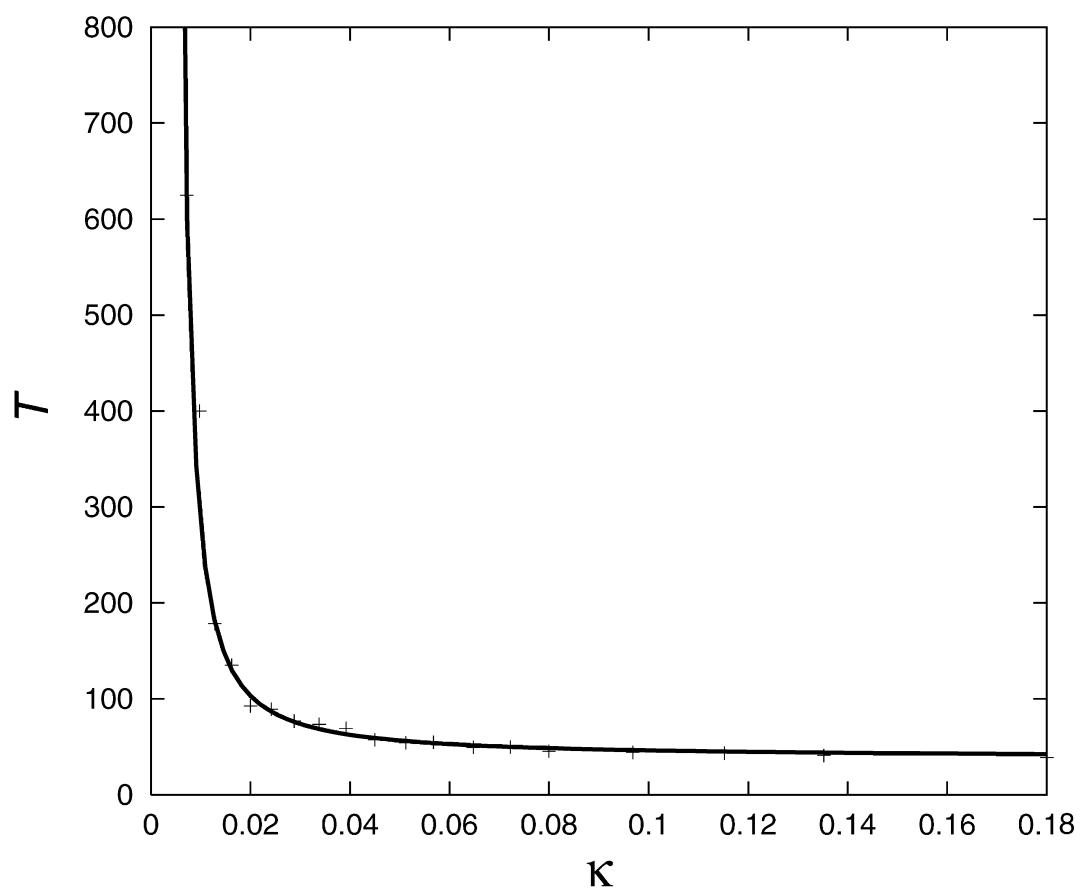

Fig. 35. The dependence of the mean period $T$ on the noise intensity $\kappa$. The solid curve plots $T=38 \exp (0.02 / \kappa)$.

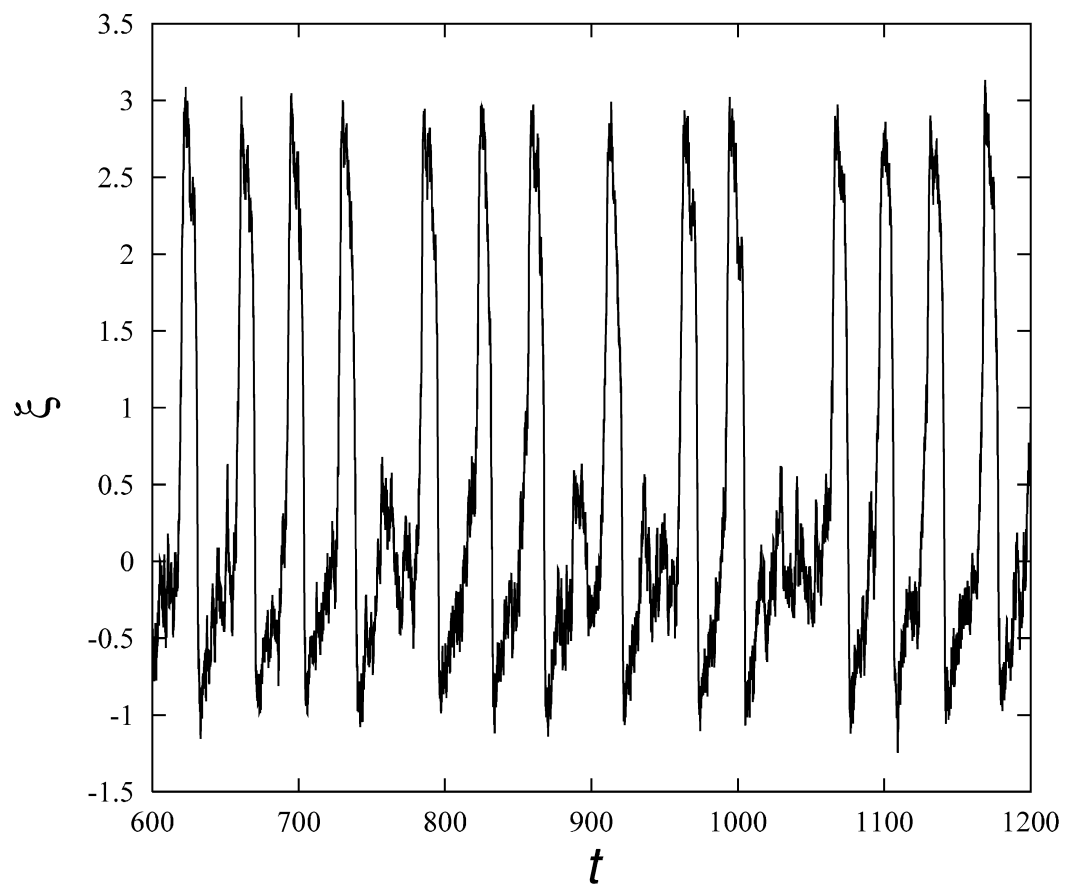

Fig. 36. Plot of $\xi(t)$ for $\kappa=0.1152$. 
It is very important that the pulses induced by noise differ little in their shape from the pulses induced by periodic force (compare Figs. 36 and 31(g)). In the first case only the pulses are somewhat noisy and the interpulse time is random. Thus do the intrinsic properties of the system reveal themselves.

\section{Conclusions}

We have demonstrated that weak noise acting upon a nonlinear dynamical system can have far-reaching consequences, such as a transition to a new state, the transformation of an unstable equilibrium state into a stable one, and vice versa, the occurrence of multistability and multimodality, noise-induced transport (stochastic ratchets), and the excitation of noise-induced oscillations. Many of these phenomena can be treated as nonequilibrium phase transitions induced by noise. The origins of these transitions lie in intersection of a certain boundary in the system phase space by the representative point under the action of noise.

\section{Appendix A. Derivation of the approximate equation for the one-dimensional probability density}

Let us consider an equation

$$
\varepsilon^{2} \ddot{x}+\dot{x}+F(x)=\xi(t),
$$

where $\xi(t)$ is white noise of zero mean and intensity $K$. Eq. (A.1) can be rewritten in the form of two equations of the first order:

$$
\varepsilon \dot{x}=y, \quad \varepsilon \dot{y}=-\frac{y}{\varepsilon}-F(x)+\xi(x, t) .
$$

The two-dimensional Fokker-Planck equation associated with Eqs. (A.2) is

$$
\varepsilon^{2} \frac{\partial w(x, y, t)}{\partial t}=-\varepsilon\left(y \frac{\partial w}{\partial x}-F(x) \frac{\partial w}{\partial y}\right)+\frac{\partial(y w)}{\partial y}+\frac{K}{2} \frac{\partial^{2} w}{\partial y^{2}} .
$$

Let us seek a solution of Eq. (A.3) in the form of the following expansion:

$$
w(x, y, t)=\sum_{n=0}^{\infty} \varepsilon^{n} w_{n}(x, t) Y_{n}(y),
$$

where $Y_{n}(y)$ are the eigenfunctions of the boundary-value problem described by the equation

$$
\frac{K}{2} \frac{\mathrm{d}^{2} Y}{\mathrm{~d} y^{2}}+\frac{\mathrm{d}(y Y)}{\mathrm{d} y}+\lambda Y=0
$$

with the boundary conditions $Y( \pm \infty)=0$. As can be easily shown, the eigenvalues of this problem $\lambda_{n}=n$, where $n=0,1,2, \ldots$, and the eigenfunctions can be expressed in terms of the Hermite 
polynomials $H_{n}(z)$ as

$$
Y_{n}(y)=\frac{(-1)^{n}}{\sqrt{\pi K 2^{n} n !}} \mathrm{e}^{-y^{2} / K} H_{n}\left(\frac{y}{\sqrt{K}}\right) .
$$

Substituting into (A.6) the expression for the Hermite polynomial we obtain

$$
Y_{n}(y)=\sqrt{\frac{K^{n-1}}{\pi 2^{n} n !}} \frac{\mathrm{d}^{n}}{\mathrm{~d} y^{n}}\left(\mathrm{e}^{-y^{2} / K}\right) .
$$

It can be shown that the functions $Y_{n}(y)$ satisfy the following orthogonality and normalization conditions:

$$
\int_{-\infty}^{\infty} \frac{Y_{n}(y) Y_{m}(y)}{Y_{0}(y)} \mathrm{d} y=\delta_{n m} .
$$

We substitute (A.4) into Eq. (A.3) taking into account the following relationships: ${ }^{12}$

$$
\begin{aligned}
& \frac{\mathrm{d} Y_{n}(y)}{\mathrm{d} y}=\sqrt{\frac{2(n+1)}{K}} Y_{n+1}(y), \quad y Y_{n}(y)=-\sqrt{\frac{K}{2}}\left(\sqrt{n+1} Y_{n+1}(y)+\sqrt{n} Y_{n-1}(y)\right), \\
& \frac{\mathrm{d}\left(y Y_{n}(y)\right)}{\mathrm{d} y}=-\left(\sqrt{(n+1)(n+2)} Y_{n+2}(y)+n Y_{n}(y)\right) .
\end{aligned}
$$

As a result, we find

$$
\begin{aligned}
\varepsilon^{2} \sum_{n=0}^{\infty} \varepsilon^{n} Y_{n} \frac{\partial w_{n}}{\partial t}= & \sum_{n=0}^{\infty} \varepsilon^{n}\left[\varepsilon \sqrt{\frac{K}{2}}\left(\sqrt{n+1} Y_{n+1}+\sqrt{n} Y_{n-1}\right) \frac{\partial w_{n}}{\partial x}\right. \\
& \left.+\varepsilon F(x) \sqrt{\frac{2(n+1)}{K}} Y_{n+1} w_{n}-n Y_{n} w_{n}\right] .
\end{aligned}
$$

Equating the terms of $Y_{n}(y)$ with the same subscripts, we obtain the following equations:

$$
\varepsilon^{2} \frac{\partial w_{n}}{\partial t}=\sqrt{\frac{K}{-}}\left(\sqrt{n} \frac{\partial w_{n-1}}{\partial x}+\varepsilon^{2} \sqrt{n+1} \frac{\partial w_{n+1}}{\partial x}\right)+F(x) \sqrt{\frac{2 n}{K}} w_{n-1}-n w_{n} .
$$

For $n \leq 4$ these equations are

$$
\begin{aligned}
& \frac{\partial w_{0}}{\partial t}=\sqrt{\frac{K}{2}} \frac{\partial w_{1}}{\partial x}, \\
& \varepsilon^{2} \frac{\partial w_{1}}{\partial t}=\sqrt{\frac{K}{2}} \frac{\partial w_{0}}{\partial x}+\varepsilon^{2} \sqrt{K} \frac{\partial w_{2}}{\partial x}+\sqrt{\frac{2}{K}} F(x) w_{0}-w_{1}, \\
& \varepsilon^{2} \frac{\partial w_{2}}{\partial t}=\sqrt{K} \frac{\partial w_{1}}{\partial x}+\varepsilon^{2} \sqrt{\frac{3 K}{2}} \frac{\partial w_{3}}{\partial x}+\frac{2}{\sqrt{K}} F(x) w_{1}-2 w_{2},
\end{aligned}
$$

${ }^{12}$ These relationships follow from the properties of Hermite polynomials. 


$$
\begin{aligned}
& \varepsilon^{2} \frac{\partial w_{3}}{\partial t}=\sqrt{\frac{3 K}{2}} \frac{\partial w_{2}}{\partial x}+\varepsilon^{2} \sqrt{2 K} \frac{\partial w_{4}}{\partial x}+\sqrt{\frac{6}{K}} F(x) w_{2}-3 w_{3} \\
& \varepsilon^{2} \frac{\partial w_{4}}{\partial t}=\sqrt{2 K} \frac{\partial w_{3}}{\partial x}+\varepsilon^{2} \sqrt{\frac{5 K}{2}} \frac{\partial w_{5}}{\partial x}+2 \sqrt{\frac{2}{K}} F(x) w_{3}-4 w_{4} .
\end{aligned}
$$

Putting in Eqs. (A.11) $w_{i}=w_{i 0}+\varepsilon^{2} w_{i 1}+\varepsilon^{4} w_{i 2}+\varepsilon^{6} w_{i 3}+\cdots(i=1,2,3, \ldots)$, we can find sequentially the functions $w_{10}, w_{11}, w_{12}, \ldots, w_{1 n}, \ldots$. The calculations show that for $n \geq 1$ these functions can be expessed as

$$
w_{1 n}=\Phi_{1}^{(n)} \frac{\partial^{2 n-2} w_{10}}{\partial x^{(2 n-2)}}+\Phi_{2}^{(n)} \frac{\partial^{2 n-3} w_{10}}{\partial x^{(2 n-3)}}+\cdots+\Phi_{2 n-1}^{(n)} w_{10},
$$

where $\Phi_{k}^{(n)}$ are functions of $F(x)$ and its derivatives,

$$
w_{10}=\sqrt{\frac{2}{K}}\left(\frac{K}{2} \frac{\partial w_{0}}{\partial x}+F(x) w_{0}\right) .
$$

Substituting

$$
w_{1}=\sum_{n=0}^{\infty} \varepsilon^{2 n} w_{1 n}
$$

into Eq. (A.12) and using the fact that

$$
w(x, t)=\int_{-\infty}^{\infty} w(x, y, t) \mathrm{d} y=w_{0}(x, t),
$$

we obtain the following equation for $w(x, t)$ :

$$
\frac{\partial w}{\partial t}=\sqrt{\frac{K}{2}} \sum_{n=0}^{\infty} \varepsilon^{2 n} \frac{\partial w_{1 n}}{\partial x} .
$$

If $\varepsilon^{2}$ is sufficiently small then series (A.19) is converged and Eq. (A.20) is the exact one-dimensional equation for the probability density $w(x, t)$. In a stationary case Eq. (A.20) becomes

$$
\sqrt{\frac{K}{2}} \sum_{n=0}^{\infty} \varepsilon^{2 n} w_{1 n}=-G,
$$

where $G$ is the probability flux. In this case the derivatives of $w_{10}$, which are contained in the expressions for $w_{1 n}$, in their turn should be expanded as power series in $\varepsilon^{2}$ :

$$
\begin{aligned}
& \frac{\partial w_{10}}{\partial x}=-\varepsilon^{2}\left[F^{\prime \prime}(x)+\varepsilon^{2}\left(\frac{3 K}{4} F^{\mathrm{IV}}(x)+2 F(x) F^{\prime \prime \prime}(x)+6 F^{\prime}(x) F^{\prime \prime}(x)\right)+\cdots\right] w_{10}, \\
& \frac{\partial^{2} w_{10}}{\partial x^{2}}=-\varepsilon^{2}\left[F^{\prime \prime \prime}(x)+\varepsilon^{2}\left(\frac{3 K}{4} F^{\mathrm{v}}(x)+2 F(x) F^{\mathrm{IV}}(x)+8 F^{\prime}(x) F^{\prime \prime \prime}(x)+6\left(F^{\prime \prime}(x)\right)^{2}\right)+\cdots\right] w_{10}, \\
& \cdots
\end{aligned}
$$




\section{References}

[1] P.S. Landa, Nonlinear Oscillations and Waves in Dynamical Systems, Kluwer, Dordrecht, 1996.

[2] W. Horsthemke, R. Lefever, Noise-Induced Transitions, Springer, Berlin, 1984. **

[3] J. Smythe, F.E. Moss, P.V.E. McClintock, Observation of a noise-induced phase transition with an analog simulator, Phys. Rev. Lett. 51 (1983) 1062-1064. *

[4] C. Van den Broeck, J.M.R. Parrondo, J. Armero, A. Hernández-Machado, Mean field model for spatially extended systems in the presence of multiplicative noise, Phys. Rev. E 49 (1994) 2639-2643. *

[5] C. Van den Broeck, J.M.R. Parrondo, R. Toral, Noise-induced nonequilibrium phase transition, Phys. Rev. Lett. 73 (1994) 3395-3398.

[6] C. Van den Broeck, J.M.R. Parrondo, R. Toral, R. Kawai, Nonequilibrium phase transitions induced by multiplicative noise, Phys. Rev. E 55 (1997) 4084-4094.

[7] P.S. Landa, A.A. Zaikin, L. Schimansky-Geier, Influence of additive noise on noise-induced phase transitions in nonlinear lattices, Chaos solitons fractals 9 (1998) 1367-1372.

[8] P.S. Landa, A.A. Zaikin, Noise-induced phase transitions in a pendulum with a randomly vibrating suspension axis, Phys. Rev. E 54 (1996) 3535-3544.

[9] P.S. Landa, A.A. Zaikin, Nonequilibrium noise-induced phase transitions in simple systems, JETP 84 (1997) 197-208.

[10] P.S. Landa, A.A. Zaikin, Noise-induced phase transitions in nonlinear oscillators, in: AIP Conference Proceedings 465, Computing Anticipatory Systems, CASYS '98, Liege, Belgium, 1998, pp. 419-433. **

[11] P.S. Landa, A. Rabinovich, Exhibition of intrinsic properties of certain systems in response to external disturbances, Phys. Rev. E, submitted for publication.

[12] M.I. Dykman, P.V.E. McClintock, R. Mannella, N.G. Stocks, Stochastic resonance for linear and nonlinear bistable systems in a periodic field, JETP Lett. 52 (1990) 780-782 (in Russian).

[13] M.I. Dykman, R. Mannella, P.V.E. McClintock, N.G. Stocks, Comment on stochastic resonance in bistable systems, Phys. Rev. Lett. 65 (1990) 2606. **

[14] F. Moss, D. Pierson, D. O'Gorman, Stochastic resonance: tutorial and update, Bifurcation and Chaos 6 (1994) 1383-1397.

[15] K. Wiesenfeld, F. Moss, Stochastic resonance and the benefits of noise: From the ice ages to crayfish and SQUIDs, Nature 373 (1995) 33-36.

[16] M.I. Dykman, D.G. Luchinsky, R. Mannella, P.V.E. McClintock, N.D. Stein, N.G. Stocks, Stochastic resonance in perspective, Nuovo Cimento D 17 (1995) 661-683.

[17] L. Gammaitoni, P. Hänggi, P. Jung, F. Marchesoni, Stochastic resonance, Rev. Mod. Phys. 70 (1998) 223-287.

[18] R.D. Astumian, F. Moss, Overview: The constructive role of noise in fluctuation driven transport and stochastic resonance, Chaos 8 (1998) 533-538. **

[19] K. Wiesenfeld, F. Jaramillo, Minireview of stochastic resonance, Chaos 8 (1998) 539-548.

[20] P. Hänggi, R. Bartussek, Brownian rectifiers: how to convert brownian motion into directed transport, in: J. Parisi, S.C. Müller, W. Zimmermann (Eds.), Nonlinear Physics of Complex Systems - Current Status and Future Trends, Lecture Notes in Physics, Vol. 476, Springer, Berlin, 1996, pp. 294-308. **

[21] R.D. Astumian, Thermodynamics and kinetics of a Brownian motor, Science 276 (1997) 917-922.

[22] F. Jülicher, A. Ajdari, J. Prost, Modeling molecular motors, Rev. Mod. Phys. 69 (1997) 1269-1281.

[23] D.G. Luchinsky, P.V.E. McClintock, M.I. Dykman, Analogue studies of nonlinear systems, Rep. Prog. Phys. 61 (1998) 889-997. **

[24] M. Bier, Brownian ratchets in physics and biology, Contemp. Phys. 38 (1997) 371-380. **

[25] L.S. Pontryagin, A.A. Andronov, A.A. Vitt, The statistical treatment of dynamical systems, Zh. Eksp. Jeor. Fiz. 3 (1933) 165-180 (in Russian); English translation: in Noise in nonlinear dynamical systems, F. Moss, P.V.E. McClintock (Eds.) (Cambridge University Press, Cambridge, 1989). ***

[26] R.L. Stratonovich, Selected Problems of Fluctuation Theory in Radioengineering (Sov. Radio, Moscow, 1961) (in Russian); English translation: Topics in the Theory of Random Noise, Vol. 1, Gordon and Breach, New York, 1963, Vol. 2, Gordon and Breach, New York, 1967. *** 
[27] P.S. Landa, R.L. Stratonovich, On the theory of fluctuational transitions of different systems from one stable state to another, Vestnik MGU (Physics and Astronomy) No 1 (1962) 33-45 (in Russian). **

[28] H.A. Kramers, Brownian motion in a field of forces and diffusion model of chemical reactions, Physica 7 (1940) 284-304. **

[29] R.L. Stratonovich, P.S. Landa, The effect of noise on a generator with hard excitation, Izv. vuzov, Radiofizika 2 (1959) 37-45 (in Russian); English translation: in Non-Linear Transformations of Stochastic Processes, Pergamon Press, Oxford, 1965, pp. 259-268.

[30] P.S. Landa, On the stability of systems with servocontrol and random action, Avtomatika i Telemekhanika 21 (1960) 36-41 (in Russian).

[31] P.S. Landa, Losses of electrons in synchrotrons due to the quantum character of the radiation, Sov. Phys. JETP 13 (1961) 789-791.

[32] R. Landauer, J.A. Swanson, Frequency factors in the thermally activated process, Phys. Rev. 121 (1961) $1668-1674$.

[33] M.I. Dykman, M.A. Krivoglaz, Theory of fluctuational transitions between stable states of a nonlinear oscillator, Sov. Phys. JETP 50 (1979) 30. **

[34] M.I. Dykman, M.A. Krivoglaz, Theory of nonlinear oscillator interacting with a medium, in: I.M. Khalatnikov (Ed.), Soviet Physics Reviews, Harwood, New York, 1984, Vol. 5, pp. 265-446.

[35] P.S. Landa, R.L. Stratonovich, Theory of intermittency, Radiophys. Quant. Electron. 30 (1987) 53-57. *

[36] P.S. Landa, Influence of noise on the transition to chaos through intermittency, Moscow Univ. Phys. Bull. 42 (1987) 19-25.

[37] A.J. Bray, A.J. McKane, Instantaneous calculation of the escape rate for activation over a potential barrier driven by colored noise, Phys. Rev. Lett. 62 (1989) 493-496.

[38] P.S. Landa, A.A. Zaikin, M.G. Rosenblum, J. Kurths, Control of noise-induced oscillations of a pendulum with a randomly vibrating suspension axis, Phys. Rev. E 56 (1997) 1465-1470.

[39] P.S. Landa, A.A. Zaikin, On-off intermittency phenomena in a pendulum with a randomly vibrating suspension axis, Chaos, Solitons and Fractals 9 (1998) 157-169.

[40] R.S. Maier, D.L. Stein, Transition-rate theory for nongradient drift fields, Phys. Rev. Lett. 69 (1992) $3691-3695$.

[41] R.S. Maier, D.L. Stein, Escape problem for irreversible systems, Phys. Rev. E 48 (1993) 931-938.

[42] M.I. Freidlin, A.D. Wentzel, Random Perturbations of Dynamical Systems, Nauka, Moscow, 1978 (in Russian); English translation: Springer, New York, Berlin, 1984.

[43] M.I. Dykman, Large fluctuations and fluctuational transitions in system driven by colored Gaussian noise - a high-frequency noise, Phys. Rev. A 42 (1990) 2020-2029.

[44] M.I. Dykman, P.V.E. McClintock, V.N. Smelyansky, N.D. Stein, N.G. Stocks, Optimal paths and prehistory problem for large fluctuations in noise-driven systems, Phys. Rev. Lett. 68 (1992) 2718-2721.

[45] S.J.B. Einchcomb, A.J. McKane, Use of Hamiltonian mechanics in systems driven by colored noise, Phys. Rev. E 51 (1995) 2974-2981.

[46] M.I. Dykman, D.G. Luchinsky, P.V.E. McClintock, V.N. Smelyansky, Corrals and critical behavior of the distribution of optimal paths, Phys. Rev. Lett. 77 (1996) 5229-5232.

[47] D.G. Luchinsky, P.V.E. McClintock, Analogue experiments on irreversibility of classical fluctuations, Nature 389 (1997) 463-466. *

[48] D.G. Luchinsky, R.S. Maier, R. Mannella, P.V.E. McClintock, D.L. Stein, Experiments on critical phenomena in a noisy exit problem, Phys. Rev. Lett. 79 (1997) 3109-3112.

[49] L.D. Landau, E.M. Lifshitz, Mechanics, Pergamon Press, Oxford, New York, 1969.

[50] Y. Pomeau, P. Manneville, Intermittent transition to turbulence in dissipative dynamical systems, Comm. Math. Phys. 74 (1980) 189-197.

[51] H.G. Schuster, Deterministic Chaos, Physic-Verlag, Weinheim, 1984.

[52] Yu.I. Neimark, P.S. Landa, Stochastic and Chaotic Oscillations, Nauka, Moscow, 1987 (in Russian); English translation: (Kluwer Academic Publishers, Dordrecht-Boston-London, 1992).

[53] J.E. Hirsch, B.A. Huberman, D.J. Scalapino, Theory of intermittency, Phys. Rev. A 25 (1982) 519-532.

[54] J.E. Hirsch, M. Nauenberg, D.J. Scalapino, Intermittency in the presence of noise: a renormalization group formulation, Phys. Lett. 87A (1982) 391-393. 
[55] B. Hu, J. Rudnick, Exact solutions to the Feigenbaum renormalization-group equations for intermittency, Phys. Rev. Lett. 48 (1982) 1645-1648.

[56] N. Platt, E.A. Spiegel, C. Tresser, On-off intermittency: a mechanism for bursting, Phys. Rev. Lett. 70 (1993) 279-282.

[57] A.S. Pikovsky, On the interaction of strange attractors, Phys. B, Conden. Matter 55 (1984) 149-154.

[58] H. Fujisaka, T. Yamada, A new intermittency in coupled dynamical systems, Prog. Theor. Phys. 74 (1985) 918-921.

[59] J.F. Heagy, N. Platt, S.M. Hammel, Characterization of on-off intermittency, Phys. Rev. E 49 (1994) 1140-1150. *

[60] A. Cenys, H. Lustfeld, Statistical properties of the noisy on-off intermittency, J. Phys. A 29 (1996) 11-20.

[61] C.S. Peskin, G.M. Odell, G.F. Oster, Cellular motions and thermal fluctuations - the Brownian ratchet, Biophys. J. 65 (1993) 316-324.

[62] K. Svoboda, C.F. Schmidt, B.J. Schnapp, S.M. Block, Direct observation of kinesin stepping by optical trapping interferometry, Nature 365 (1993) 721-727.

[63] M.O. Magnasco, Forced thermal ratchets, Phys. Rev. Lett. 71 (1993) 1477-1481. *

[64] R.D. Astumian, M. Bier, Fluctuation driven ratchets: molecular motors, Phys. Rev. Lett. 72 (1994) 1766-1769.

[65] C.R. Doering, Randomly rattled ratchets, Nuovo Cimento Soc. Ital. Fiz. 17D (1995) 685-697.

[66] R.D. Astumian, M. Bier, Mechanochemical coupling of the motion of molecular motors to ATP hydrolysis, Biophys. J. 70 (1996) 637-653.

[67] L.P. Faucheux, L.S. Bourdieu, P.D. Kaplan, A.J. Libchaber, Optical thermal ratchet, Phys. Rev. Lett. 74 (1995) 1504-1507.

[68] A. Ajdari, J. Prost, Movement induit par un potential de basse symétrie: dielectrophorèse pulsée, C. R. Acad. Sci. Paris 315 (1992) 1635-1639.

[69] L. Gorre-Talini, J.P. Spatz, P. Silberzan, Dielectrophoretic ratchets, Chaos 8 (1998) 650-656.

[70] R.P. Feynman, R.B. Leighton, M. Sands, The Feynman Lectures on Physics, Vol. 1, Addison-Wesley, Reading, MA, Palo Alto, London, 1963 (Chapter 46). ***

[71] M. von Smoluchowski, Experimentell Nachweisbare der ublichen Thermodynamik wiedersprechende Molekularphanomene, Physik. Zeitschr. 13 (1912) 1069-1080.**

[72] L. Brillouin, Can the rectifier become a thermodynamical demon? Phys. Rev. 78 (1950) 627-628.

[73] D.K.C. MacDonald, Brownian movement, Phys. Rev. 108 (1957) 541-545.

[74] C.T.J. Alkemade, On the problem of Brownian motion of non-linear systems, Physica 24 (1958) 1029-1034.

[75] N.G. Van Kampen, Thermal fluctuations in a nonlinear system, Phys. Rev. 110 (1958) 319-323.

[76] A. Marek, A note to recent theories of Brownian motion in non-linear systems, Physica 25 (1959) 1358-1367.

[77] R.L. Stratonovich, On the paradox in the theory of thermal fluctuations of nonlinear resistors, Vestnik MGU No. 4 (1960) 99-102 (in Russian). **

[78] R.L. Stratonovich, Nonlinear Nonequilibrium Thermodynamics, Nauka, Moscow, 1985 (in Russian); English translation: Springer, Berlin, 1992, pp. 80-81. **

[79] C.R. Doering, W. Horsthemke, J. Riordan, Nonequilibrium fluctuation-induced transport, Phys. Rev. Lett. 72 (1994) 2984-2987.

[80] L. Schimansky-Geier, M. Kschicho, T. Fricke, Flux of particles in sawtooth media, Phys. Rev. Lett. 79 (1997) 3335-3338.

[81] R. Bartussek, P. Hänggi, J.G. Kissner, Periodically rocked thermal ratchets, Europhys. Lett. 28 (1994) $459-464$.

[82] M. Millonas, M.I. Dykman, Transport and current reversal in stochastically driven ratchets, Phys. Lett. A 185 (1994) 65-69.

[83] M. Bier, Reversals of noise induced flow, Phys. Lett. A 211 (1996) 12-18.

[84] M. Arrayás, P.V.E. McClintock, N.D. Stein, R. Mannella, A.J. McKane, Current reversals in a ratchet driven by quasimonochromatic noise, in: C. Claeys, E. Simoen (Eds.), Noise in Physical Systems and 1/f Fluctuations, World Scientific, Singapore, 1997, pp 381-384. *

[85] R. Bartussek, P. Hänggi, B. Lindner, L. Schimansky-Geier, Ratchets driven by harmonic and white noise, Physica D 109 (1997) 17-23.

[86] P.S. Landa, Noise-induced transport of Brownian particles with consideration for their mass, Phys. Rev. E 58 (1998) 1325-1333. 
[87] I.I. Blekhman, Vibrational Mechanics, Nauka, Moscow, 1994 (in Russian).

[88] G.E. Uhlenbeck, L.S. Ornstein, On the theory of Brownian motion, Phys. Rev. 36 (1930) 823-841.

[89] I.I. Fedchenia, Boundary stochastic problems, multistability in the presence of fluctuations and noice-induced phase transitions, Physica A 125A (1984) 577-590.

[90] M.I. Dykman, R. Mannella, P.V.E. McClintock, N.G. Stocks, Fluctuation-induced transitions between periodic attractors: Observation of supernarrow spectral peaks near a kinetic phase transition, Phys. Rev. Lett. 65 (1990) 48-51.

[91] M.I. Dykman, D.G. Luchinsky, R. Mannella, P.V.E. McClintock, N.D. Stein, N.G. Stocks, Supernarrow spectral peaks and high-frequency stochastic resonance in systems with coexisting periodic attractors, Phys. Rev. E 49 (1994) 1198-1215.

[92] S.D. Robinson, F.E. Moss, P.V.E. McClintock, Experimental observation of stochastic postponements of critical onsets in a bistable system, J. Phys. A 18 (1985) L89-94.

[93] P.L. Kapitsa, Dynamic stability of a pendulum with a vibrating suspension axis, ZhETF 21 (1951) 588-597 (in Russian).

[94] P.L. Kapitsa, Pendulum with vibrated suspension, Uspekhi pizicheskikh nauk 44 (1951) 7-20 (in Russian).

[95] R.L. Stratonovich, Yu.M. Romanovsky, Parametrical action of a random force upon linear and nonlinear oscillatory systems, Nauchnye doklady vysshey shkoly, ser. fiz.-mat. 3 (1958) 221-226 (in Russian).

[96] E.T. Whittaker, G.N. Watson, A Course of Modern Analysis, Cambridge University Press, Cambridge, 1927.

[97] Ch. Kurrer, K. Schulten, Effect of noise and perturbations on limit cycle systems. Physica D 50 (1991) 311-320.

[98] W.P. London, J.A. Yorke, Recurrent outbreaks of measles, chickenpox and mumps, I: Seasonal variation in contact rates, Am. J. Epidem. 98 (1973) 453-468.

[99] L.F. Olsen, H. Degn, Chaos in biological systems, Quart. Rev. Biophys. 18 (1985) 165-225.

[100] K. Dietz, The incidence of infectious diseases under the influence of seasonal fluctuations, Lect. Notes Biomath. 11 (1976) 1-15.

[101] L.F. Olsen, W.M. Schaffer, Chaos versus noisy periodicity: alternative hypothesis for childhood epidemics, Science 249 (1990) 499-504.

[102] R. Engbert, F.R. Drepper, Chance and chaos in population biology - models of recurrent epidemics and food chain dynamics, Chaos, Solitons Fractals 4 (1994) 1147-1169.

[103] P.S. Landa, A.A. Zaikin, Random and chaotic oscillations in a model of childhood epidemics caused by seasonal variations of the contact rate, in: Applied nonlinear dynamics and stochastic systems near the millenium, AIP Conference Proceedings 411, San Diego, CA, 1997, pp. 321-326.

[104] K.F. Bonhoeffer, Über die Aktivierung von passiven Eisen in Salpetersäure, Z. Elektrochem. 47 (1941) $147-156$.

[105] K.F. Bonhoeffer, Activation of passive iron as a model for the excitation of nerve, J. Gen. Physiol. 32 (1948) 69-75.

[106] K.F. Bonhoeffer, G. Langhammer, Über periodische Reaktionen. IV. Theorie der kathodischen polarisation von Eisen in Saltpetersäure, Z. Elektrochem. 52 (1948) 67-72.

[107] K.F. Bonhoeffer, Modelle der Nervenerregung, Naturwissenschaften 40 (1953) 301-311.

[108] B. van der Pol, On relaxation oscillation, Philos. Mag. 2 (1926) 978-992.

[109] R. Fitz Hugh, Impulses and physiological states in theoretical models of nerve membranes, Biophys. J. 1 (1961) 445-452.

[110] J. Nagumo, S. Arimoto, S. Yoshizawa, An active pulse transmission line simulating nerve axon, Proc. IRE 50 (1962) 2061-2070.

[111] R. Fitz Hugh, Mathematical models of excitation and propagation in nerve, in: H.P. Schwan (Ed.), Biological Engineering, McGraw-Hill, New York, 1969, pp. 1-85.

[112] M. Okuda, A new method of nonlinear analysis for threshold and shaping action in transient states, Prog. Theor. Phys. 66 (1981) 90-100.

[113] S. Rajasekar, M. Lakshmanan, Period-doubling bifurcations, chaos, phase-locking and devil's staircase in a Bonhoeffer-van der Pol oscillator, Physica D 32 (1988) 146-152.

[114] A. Rabinovitch, I. Rogachevskii, Threshold, excitability and isochrones in the Bonhoeffer-van der Pol system, Chaos, Solitons and Fractals, submitted for publication. 
[115] H. Treutlein, K. Schulten, Noise-induced limit cycles of the Bonhoeffer-van der Pol model of neural pulses, Ber. Bunsenges. Phys. Chem. 89 (1985) 710-718.

[116] W.P. Wang, Interaction of adiabatic phantom attractors, Nonlinearity 9 (1996) 739-759.

[117] S. Yasin, M. Friedman, S. Goshen, A. Rabinovitch, R. Thieberger, Intermittency and phase locking of the Bonhoeffer-van der Pol model, J. Theor. Biol. 160 (1993) 179-184.

[118] A. Rabinovitch, R. Thieberger, M. Friedman, S. Goshen, Resonance effects in the Bonhoeffer-van der Pol system, Chaos Solitons Fractals 7 (1996) 1713-1719.

[119] H. Treutlein, K. Schulten, Noise-induced neural impulses, Eur. Biophys. J. 13 (1986) 355-365. 